\title{
Development of RWHet to Simulate Contaminant Transport in Fractured Porous Media
}

\author{
prepared by \\ Yong Zhang, Eric M. LaBolle, Donald M. Reeves, and Charles Russell
}

submitted to

Nevada Site Office

National Nuclear Security Administration

U.S. Department of Energy

Las Vegas, Nevada

July 2012

PUBLICATION \#45244 
Reference herein to any specific commercial product, process, or service by trade name, trademark, manufacturer, or otherwise, does not necessarily constitute or imply its endorsement, recommendation, or favoring by the United States Government or any agency thereof or its contractors or subcontractors.

Available for sale to the public from:

U.S. Department of Commerce

National Technical Information Service

5301 Shawnee Road

Alexandria, VA 22312

Phone: 800.553.6847

Fax: 703.605.6900

Email: orders@ntis.gov

Online ordering: http://www.osti.gov/ordering.htm

Available electronically at http://www.osti.gov/bridge

Available for a processing fee to the U.S. Department of Energy and its contractors, in paper, from:

U.S. Department of Energy

Office of Scientific and Technical Information

P.O. Box 62

Oak Ridge, TN 37831-0062

Phone: 865.576.8401

Fax: 865.576.5728

Email: reports@adonis.osti.gov 


\title{
Development of RWHet to Simulate Contaminant Transport in Fractured Porous Media
}

\author{
prepared by \\ Yong Zhang, Eric M. LaBolle, Donald M. Reeves, and Charles Russell \\ Division of Hydrologic Sciences, Desert Research Institute \\ Nevada System of Higher Education
}

Publication No. 45244

submitted to

Nevada Site Office

National Nuclear Security Administration

U.S. Department of Energy

Las Vegas, Nevada

July 2012

The work upon which this report is based was supported by the U.S. Department of Energy under Contract \#DE-NA0000939. Approved for public release; further dissemination unlimited. 
THIS PAGE INTENTIONALLY LEFT BLANK 


\section{EXECUTIVE SUMMARY}

Accurate simulation of matrix diffusion in regional-scale dual-porosity and dualpermeability media is a critical issue for the DOE Underground Test Area (UGTA) program, given the prevalence of fractured geologic media on the Nevada National Security Site (NNSS). Contaminant transport through regional-scale fractured media is typically quantified by particletracking based Lagrangian solvers through the inclusion of dual-domain mass transfer algorithms that probabilistically determine particle transfer between fractures and unfractured matrix blocks. UGTA applications include a wide variety of fracture aperture and spacing, effective diffusion coefficients ranging four orders of magnitude, and extreme end member retardation values.

This report incorporates the current dual-domain mass transfer algorithms into the wellknown particle tracking code RWHet [LaBolle, 2006], and then tests and evaluates the updated code. We also develop and test a direct numerical simulation (DNS) approach to replace the classical transfer probability method in characterizing particle dynamics across the fracture/matrix interface. The final goal of this work is to implement the algorithm identified as most efficient and effective into RWHet, so that an accurate and computationally efficient software suite can be built for dual-porosity/dual-permeability applications. RWHet is a mature Lagrangian transport simulator with a substantial user-base that has undergone significant development and model validation. In this report, we also substantially tested the capability of RWHet in simulating passive and reactive tracer transport through regional-scale, heterogeneous media.

Four dual-domain mass transfer methodologies were considered in this work. We first developed the empirical transfer probability approach proposed by Liu et al. [2000], and coded it into RWHet. The particle transfer probability from one continuum to the other is proportional to the ratio of the mass entering the other continuum to the mass in the current continuum. Numerical examples show that this method is limited to certain ranges of parameters, due to an intrinsic assumption of an equilibrium concentration profile in the matrix blocks in building the transfer probability. Subsequently, this method fails in describing mass transfer for parameter combinations that violate this assumption, including small diffusion coefficients (i.e., the freewater molecular diffusion coefficient $D_{0} \leq 1 \times 10^{-11}$ meter $^{2} /$ second), relatively large fracture spacings $2 B$ (such as $2 B \geq 1$ meter), and/or relatively large matrix retardation coefficients $R_{m}$ (i.e., $R_{m} \geq 2$ ). These "outliers” in parameter range are common in UGTA applications.

To address the above limitations, we then developed a Direct Numerical Simulation (DNS)-Reflective method. The novel DNS-Reflective method can directly track the particle dynamics across the fracture/matrix interface using a random walk, without any empirical assumptions. This advantage should make the DNS-Reflective method feasible for a wide range of parameters. Numerical tests of the DNS-Reflective, however, show that the method is computationally very demanding, since the time step must be very small to resolve particle transfer between fractures and matrix blocks.

To improve the computational efficiency of the DNS approach, we then adopted Roubinet et al.'s method [2009], which uses first passage time distributions to simulate dualdomain mass transfer. The DNS-Roubinet method was found to be computationally more efficient than the DNS-Reflective method. It matches the analytical solution for the whole range of major parameters (including diffusion coefficient and fracture aperture values that are 
considered “outliers” for Liu et al.'s transfer probability method [2000]) for a single fracture system. The DNS-Roubinet method, however, has its own disadvantage: for a parallel fracture system, the truncation of the first passage time distribution creates apparent errors when the fracture spacing is small, and thus it tends to erroneously predict breakthrough curves (BTCs) for the parallel fracture system.

Finally, we adopted the transient range approach proposed by Pan and Bodvarsson [2002] in RWHet. In this method, particle transfer between fractures and matrix blocks can be resolved without using very small time steps. It does not use any truncation of the first passage time distribution for particles. Hence it does not have the limitation identified above for the DNS-Reflective method and the DNS-Roubinet method. Numerical results were checked against analytical solutions, and also compared to DCPTV2.0 [Pan, 2002]. This version of RWHet (called RWHet-Pan\&Bodvarsson in this report) can accurately capture contaminant transport in fractured porous media for a full range of parameters without any practical or theoretical limitations. 


\section{CONTENTS}

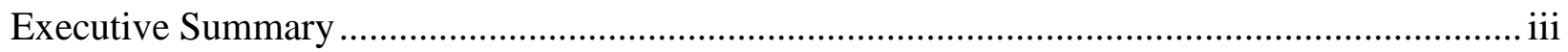

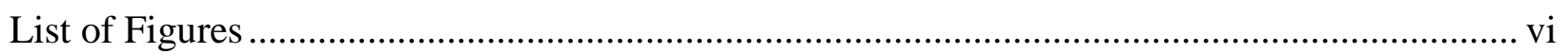

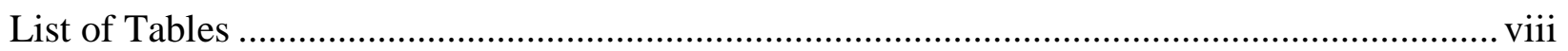

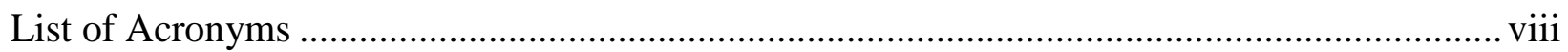

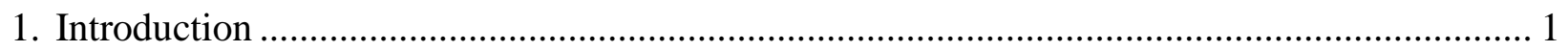

2. RWHet combined with the transfer probability method developed by Liu et al. [2000].......... 1

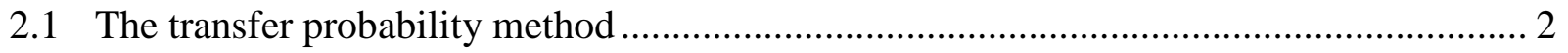

2.2 Numerical experiments of Liu et al.'s method [2000] ................................................. 3

2.3 Fracture/Matrix properties at the Rainer Mesa site ................................................... 8

3. The direct numerical simulation (DNS) of contaminant transport in fractured porous

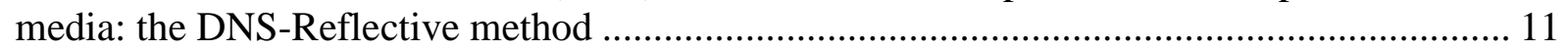

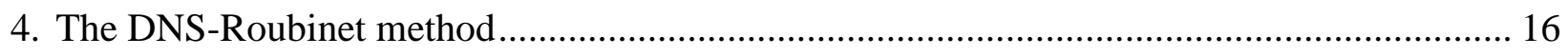

5. The transient range approach proposed by Pan and Bodvarsson [2002] .............................. 19

5.1 Methodology of Pan and Bodvarsson [2002] ........................................................ 19

5.2 Numerical examples of RWHet-Pan\&Bodvarsson .................................................... 21

5.2.1. Test $1:{ }^{3} \mathrm{H}$ with variable free-water diffusion coefficient $\mathrm{D}_{0}$ and fracture spacing

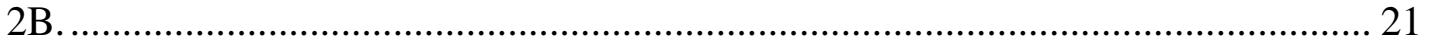

5.2.2 Test 2: Variable fracture spacing 2B (from $0.1 \mathrm{~m}$ to $20 \mathrm{~m}$ )................................... 25

5.2.4. Test 4: Variable fracture aperture $2 \mathrm{~b}$ (from $2 \mathrm{~b}=2 \times 10^{-5} \mathrm{~m}$ to $2 \mathrm{~b}=1 \times 10^{-3} \mathrm{~m}$ ) ........... 28

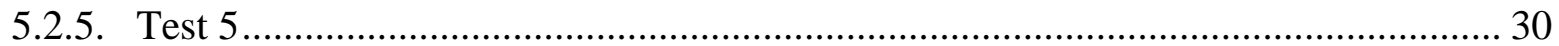

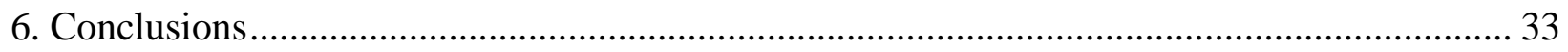

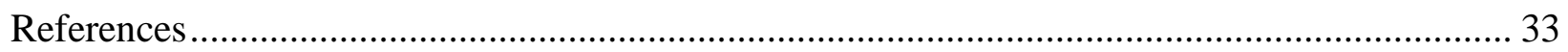

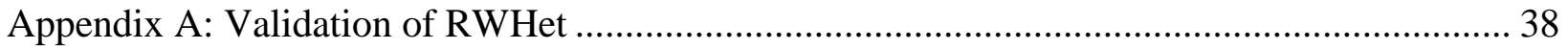

A.1. Validation of RWHet using one-dimensional transport tests: Advection, dispersion,

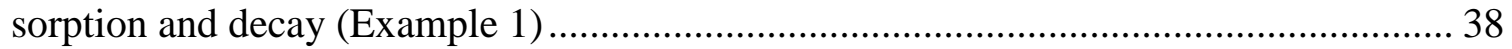

A.2. Two-dimensional homogeneous box: Transport in a radial flow field (Examples 2

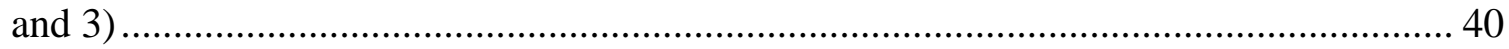

A.3. Two-dimensional heterogeneous box: Advection-only transport (Examples 4 and 5).... 45

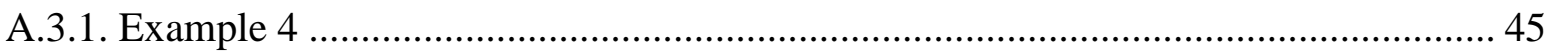

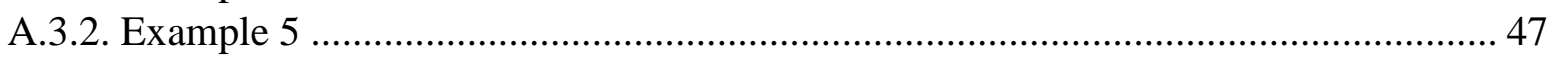

A.4. Two-dimensional heterogeneous box: Advection \& dispersion (Examples 4 and 5)....... 49

A.5. Walkabout Verification Test 3 (Example 6) ................................................................. 51 


\section{LIST OF FIGURES}

1. Numerical tests of RWHet3.25_NUFT.f90: Variable free-water diffusion coefficient $D_{0}$ - the simulated breakthrough curves in fracture continuum using RWHet3.25_NUFT.f90 (symbols) versus analytical solutions (lines).

2. Numerical tests of RWHet3.25_NUFT.f90: A small free-water diffusion coefficient $D_{0}=1 \times 10^{-11}$ meter $^{2} /$ second - the simulated breakthrough curves in fracture continuum using RWHet3.25_NUFT.f90 (symbols) versus analytical solutions (lines).. ....................... 4

3. Numerical tests of RWHet3.25_NUFT.f90: Variable aperture $2 b$ (units: meter).................... 5

4. Numerical tests of RWHet3.25_NUFT.f90: Variable longitudinal dispersivity $\alpha_{\mathrm{L}}$ in fracture (units: meter).

5. Numerical tests of RWHet3.25_NUFT.f90: Variable matrix retardation coefficient $R_{m}$. ..... 6

6. Numerical tests of RWHet3.25_NUFT.f90: Variable fracture retardation coefficient $R_{f}$. .... 6

7. Numerical tests of RWHet3.25_NUFT.f90: Influence of fracture spacing on the BTC: analytical solutions (lines) versus particle-tracking results of RWHet3.25_NUFT.f90 (symbols).

8. The simulated solution (blue symbols) versus the analytical solution (the red line) of tracer breakthrough curves of RWHet3.25_NUFT.f90 for RM site model parameters (see the text for the value of each parameter).

9. The simulated solution using RWHet3.25_NUFT.f90 (blue symbols) versus the analytical solution (the red line) of tracer breakthrough curves, where the model parameters are the same as Figure 7, except for the matrix retardation coefficient $R_{m}$. ...... 10

10. Conceptual model of a single fracture-matrix system.

11. DNS-Reflective method: Numerical examples of the one-side reflection scheme: the simulated results (symbols) versus the analytical solute concentration distributions (lines, from Carslaw and Jaeger [1959]) at $t=5$ for a composite media with discrete diffusion coefficients and effective porosity.

12. DNS-Reflective method: Influence of (half) aperture $b$ (left figures) and the free-water diffusion coefficient $D_{0}$ (right figures) on the breakthrough curve for the fracture at depth $z=0.666 \mathrm{~m}$ : the DNS-Reflective solutions (lines) versus the analytical solutions (symbols, Tang et al. [1981]).

13. A snapshot (particle clouds) simulated by the DNS-Reflective method in a single fracture with downward transport and fracture-matrix particle transfer.

14. DNS-Roubinet solutions (symbols) versus the analytical solutions (lines) [Tang et al., 1981] for a single fracture with variable fracture aperture $b$ (a) and diffusion coefficient $D_{0}$ (b).

15. DNS-Roubinet solutions of the tracer breakthrough curves (symbols) versus the analytical solutions (lines) [Tang et al., 1981] for single fracture at various depths (denoted as " $\mathrm{"}$ " in the legend) in the fracture. 
16. DNS-Roubinet solutions of the tracer breakthrough curves (symbols) versus the analytical solutions (lines) [Sudicky and Frind, 1982] for parallel fracture at various depths in the fracture.

17. The RWHet-Pan\&Bodvarsson solutions (symbols) versus the analytical solution (black line) and the solution of DCPTV2.0 (green line).....

18. Tests of RWHet-Pan\&Bodvarsson: Influence of the free-water molecular diffusion coefficient $D_{0}$ on BTC.

19. Tests of RWHet-Pan\&Bodvarsson: $\left({ }^{3} \mathrm{H}\right.$ with various $\left.D\right)$ Influence of the free-water molecular diffusion coefficient $D_{0}$ on BTC.

20. Tests of RWHet-Pan\&Bodvarsson: The smallest $D_{0}\left(=2.40 \times 10^{-11} \mathrm{~m}^{2} / \mathrm{second}\right)$ (free-water diffusion coefficient) for ${ }^{3} \mathrm{H}: R=1$, aperture $2 b=2 \times 10^{-5} \mathrm{~m}$, and spacing $2 B=0.1 \mathrm{~m}$ (top plots) or $0.5 \mathrm{~m}$ (bottom plots).

21. Tests of RWHet-Pan\&Bodvarsson: Influence of the fracture spacing on BTC. The right plot is the log-log version of the left plot. $R f=1, R m=30$, aperture $2 b=2 \times 10^{-5} \mathrm{~m}$, and the free-water diffusion coefficient $D_{0}=2.50 \times 10^{-11} \mathrm{~m}^{2} /$ second.

22. Tests of RWHet-Pan\&Bodvarsson: Influence of the fracture spacing on BTC. The right plot is the log-log version of the left plot. $R f=1, R m=30$, aperture $2 b=2 \times 10^{-5} \mathrm{~m}$, and the free-water diffusion coefficient $D_{0}=2.50 \times 10^{-11} \mathrm{~m}^{2} /$ second.

23. Tests of RWHet-Pan\&Bodvarsson: Influence of the retardation coefficient on BTC........... 28

24. Tests of RWHet-Pan\&Bodvarsson: Influence of the fracture aperture on BTC.

25. Tests of RWHet-Pan\&Bodvarsson: Updated version of Figure 7: The simulated solution (blue symbols) versus the analytical solution (the red line) of tracer breakthrough curves, where the model parameters are the same as Figure 7.

26. Tests of RWHet-Pan\&Bodvarsson: Updated version of Figure 8: The numerical solution (blue symbols) versus the analytical solution (the red line) of tracer breakthrough curves, where the model parameters are the same as Figure 8, except for the matrix retardation coefficient $R_{m}$.

27. Tests of RWHet-Pan\&Bodvarsson: (non-sorbing radionuclide subject with a 4 order magnitude in velocity $\mathrm{V}$ ) show that the RfVf correction is insensitive to velocity. The right plot is the semi$\log$ version of the left plot. Retardation coefficient $R=1$, aperture $2 b=2 \times 10^{-5} \mathrm{~m}$, the free-water

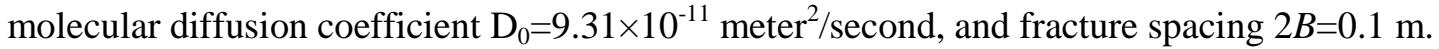

28. Example 1 - Comparison of the tracer snapshot at time $\mathrm{t}=2000$ days using RWHet (symbols) and the analytical solutions (red lines).

29. Example 2 - (a) shows the contour of hydraulic heads calculated by MODFLOW. (c) shows RWHet solutions of particle plumes at various times. The streamlines are shown in (b). The meshes at the background represent the actual model meshes ( 31 columns $\times$ 31 rows). The pumping well is located at the middle grid of each figure.

30. Example 2 - Two-dimensional transport in a radial flow field.

31. Example 3 - Concentration profiles (i.e., normalized concentration over time) at the well during an injection/pumping cycle. 
32. Example 3 - Particle positions (due to the advection-only transport) at various times during the injection cycle (a) and the pumping cycle (b).

33. Example 4 - (a) Configuration of the flow model in a heterogeneous aquifer with wells. (b), (c) and (d) show the simulated streamlines (black lines) using RWHet, due to various injection and pumping rates.

34. Example 5 - Particle tracking through a heterogeneous medium.

35. Example 5 - Statistics of particle tracking simulation (advection-only) using RWHet. (a) and (b) show the mean and variance of displacement for the 9 particles along the longitudinal direction.

36. Example 4 - Distribution of solute concentration at time $t=36.5$ days as solved by

RWHet. .

37. Example 5 - Time evolution of solute particles (snapshots) through the strong heterogeneous flow field (same as Figure 33). 50

38. Example 6 - Left: The contour of the hydraulic heads simulated by MODFLOW (black lines) and the streamlines calculated by RWHet (red lines).

\section{LIST OF TABLES}

1. Computed transfer probabilities for various matrix retardation coefficients......................... 9

2. The six numerical cases shown in Figure 12 and the time step for particle tracking. 15

\section{LIST OF ACRONYMS}

DOE U.S. Department of Energy

DNS Direct Numerical Simulation

NNSS Nevada National Security Site

RM Rainier Mesa

RWHet Random Walk in Heterogeneous media [LaBolle, 2006]

UGTA Underground Test Area 


\section{INTRODUCTION}

Fractured media are ubiquitous. The lack of analytical solutions for contaminant transport through fractured rock masses motivated the development of numerical methods. Specific software suites including for example FEHM [Zyvoloski, 2007], SLIM-FAST [Maxwell and Tompson, 2006], and DCPT [Pan, 2002] were designed using the particletracking approach to capture solute dynamics through regional-scale fractured media. The fully Lagrangian methods are superior to the standard Eulerian solvers in simulating transport in regional-scale fractured media, due to computational efficiency [LaBolle et al., 1996; Liu et al., 2000], sub-flow-grid scale resolution of concentrations, and the construction of a sample path for the underlying stochastic process.

The Lagrangian solver has been used widely by hydrologists for more than three decades. It was first applied to tracer transport in saturated alluvial formations [Ahlstrom et al., 1977; Prickett et al., 1981], and then porous media of all types [Uffink, 1985; Kinzelbach, 1988; Cordes et al., 1991; Mahinthakumar and Valocchi, 1992; Tompson, 1993; Semra et al., 1993; LaBolle et al., 1996, 1998, 2000; LaBolle and Fogg, 2001; Weissmann et al., 2002; Maxwell et al., 2007; Green, 2010] and extended to fractured rocks with matrix diffusion [Yamashita and Kimura, 1990; Wels et al., 1997; Liu et al., 2000; Tsang and Tsang, 2001; Roubinet et al., 2009, 2012].

We applied/developed four different methods to capture particle transport through fracture/matrix media. These methods include 1) the transfer probability approach proposed by Liu et al. [2000], 2) the DNS (direct numerical simulation)-Reflective method proposed by this study, 3) the DNS-Roubinet method using the first passage time distribution proposed by Roubinet et al. [2009], and 4) the transient activity range method proposed by Pan and Bodvarsson [2002]. Each method will be introduced in detail in the next sections, followed by numerical tests and comparisons. The best method is identified and implemented into the well-known particle tracking code RWHet [LaBolle, 2006], and then tested extensively.

RWHet was selected as the hosting software because 1) it has been demonstrated to be an accurate, computationally efficient particle-tracking approach [see for example, LaBolle et al., 1996, 1998, 2000; among many others], 2) it has been used widely by numerical modelers in the hydrology community, and 3) it can read directly the water flux or hydraulic head outputs generated by Eulerian-based flow models, such as MODFLOW, or other forms of velocity fields. We have also extensively tested RWHet (see Appendix A), where numerical examples show that RWHet can capture efficiently and accurately the transport of both passive and reactive tracers through regional-scale heterogeneous media with abrupt interfaces of transport properties (such as velocity and dispersion coefficient).

\section{RWHet COMBINED WITH THE TRANSFER PROBABILITY METHOD DEVELOPED BY LIU ET AL. [2000]}

We first applied the original transfer probability method proposed by Liu et al. [2000] into RWHet. In the following (subsection 2.1), we introduce the methodology first, and then extensively test the capability of the updated code RWHet in subsections 2.2 and 2.3 . 


\subsection{The transfer probability method}

In the dual-continuum model, the particle transfer probability from one continuum to the other can be calculated as the ratio of the mass entering the other continuum during the time interval to the mass in the current continuum at the beginning of this time interval [Pan et al., 2001, page 8]. Liu et al. [2000, Eqs. (16) and (17)] proposed the following two transfer probabilities

$$
\begin{gathered}
P_{f m}=\frac{F_{f m} \Delta t}{V_{f} C_{f}}=\left[\frac{Q_{f m}}{V_{f}}+\frac{2 D_{f m} A}{V_{f}(1-\lambda) S}\right] \Delta t, \\
P_{m f}=\frac{F_{m f} \Delta t}{V_{m} C_{m}}=\frac{2 D_{f m} A}{V_{m}(1-\lambda) S} \Delta t,
\end{gathered}
$$

when the water flow rate from fractures to the matrix within the block (denoted as $Q_{f m}$ $\left[\mathrm{m}^{3} \mathrm{~s}^{-1}\right]$ ) is positive (i.e., water flows from fractures to the matrix). Here $F_{f m}\left[\mathrm{~kg} \mathrm{~s}^{-1}\right]$ is the transport rate (due to both advection and dispersion) from fractures to the matrix; $F_{m f}$ is the transport rate (due to both advection and dispersion) from the matrix to fractures; $V_{f}$ (or $V_{m}$ ) $\left[\mathrm{m}^{3}\right]$ is the liquid volume within the fracture (or matrix) continuum (defined as the grid block volume multiplied by the porosity); $C_{f}$ (or $C_{m}$ ) $\left[\mathrm{kg} \mathrm{m}^{-3}\right]$ is the solute concentration in fractures (or the matrix); $D_{f m}\left[\mathrm{~m}^{2} \mathrm{~s}^{-1}\right]$ is the hydrodynamic dispersion coefficient for solute transport between fractures and the matrix; $A\left[\mathrm{~m}^{2}\right]$ is the fracture-matrix interface area available for solute transport between the continua within the block; $S[\mathrm{~m}]$ denotes the distance from the fracture-matrix interface to the center of the matrix; and $\lambda$ is the shape function and $\lambda=1 / 3$ for the layered matrix with a finite thickness (here the term "layered" denotes the shape of matrix blocks, see also the same definition used by Crank [1975] and Haggerty et al. [2000]).

The transfer probability defined by (1a) and (1b) can be related to fracture/matrix properties. Here the total water volume in fractures within each grid $\left(V_{f}\right)$ can be defined as

$$
V_{f}=(d x d y d z) \theta_{f}
$$

where $d x, d y$, and $d z[\mathrm{~m}]$ are the size of the grid along $X, Y$, and $Z$ directions, respectively; and $\theta_{f}$ [dimensionless] denotes the fracture porosity (i.e., the ratio of the fluid volume within the fractures to the total grid volume).

Similarly, $V_{m}$ is

$$
V_{m}=\left(1-\theta_{f}\right)(d x d y d z) \theta_{m},
$$

where $\theta_{m}$ [dimensionless] denotes the matrix porosity.

The contact area between fracture and matrix, $A$, is defined as:

$$
A=\frac{d x}{B} d y d z
$$

where $B[\mathrm{~m}]$ is the (half) fracture spacing. 
Substituting (2) and (4) into (1a), we obtain the probability for a fracture particle to enter the matrix during the time interval $\Delta t$ :

$$
P_{f m}=\left[\frac{Q_{f m}}{(d x d y d z) b R_{f} / B}+\frac{3 D^{*}}{S b R_{m} R_{f}}\right] \Delta t,
$$

where $D^{*}=D_{f m} R_{m}, R_{m}$ [dimensionless] is the matrix retardation coefficient, $b$ [m] is the (half) fracture aperture, and $R_{f}$ [dimensionless] is the retardation coefficient in fractures. Note here $R_{f}$ is added in Eq. (5) for the case of sorption, as also shown by Eq. (30) in Liu et al. [2000]. Substituting (3) and (4) into (1b), we get the other transfer probability (i.e., from the matrix to the adjacent fracture)

$$
P_{m f}=\frac{3 D^{*}}{S^{2} R_{m}^{2} \theta_{m}} \Delta t .
$$

Probability (6) is the same as the one used by Maxwell and Tompson [2006, Eq. (B23b)]. The transfer probability from fractures to the matrix, as expressed by (5), however, is slightly different from the following one used by Maxwell and Tompson [2006, Eq. (B23a)]:

$$
P_{f m}=\frac{3 D^{*}}{S b R_{m}} \Delta t .
$$

Note that Maxwell and Tompson [2006] did not consider advective flux between fractures and the matrix, and mass exchange occurs only because of diffusion into and out of the matrix blocks. In the following we will show that the formula (5) is valid for the case of variable retardation coefficients for fractures.

\subsection{Numerical experiments of Liu et al.'s method [2000]}

The above methodology is coded into RWHet. The resultant code is called RWHet3.25_NUFT.f90. Note here the word "NUFT" is used since a preceding version of RWHet reads dual-permeability flow fields generated by the numerical flow code NUFT [Nitao, 2001]; however, the algorithm developed below is not restricted to NUFT flow fields and valid for both dual-porosity and dual-permeability flow fields from any finite-difference flow code.

Numerical results using RWHet3.25_NUFT.f90 are compared with analytical solutions given in Sudicky and Frind [1982]. Almost all parameters are tested, including diffusion coefficient (Figure 1 and Figure 2), aperture (Figure 3), longitudinal dispersivity within the fractures (Figure 4), matrix retardation coefficient (Figure 5), fracture retardation coefficient (Figure 6), and fracture spacing (Figure 7). 


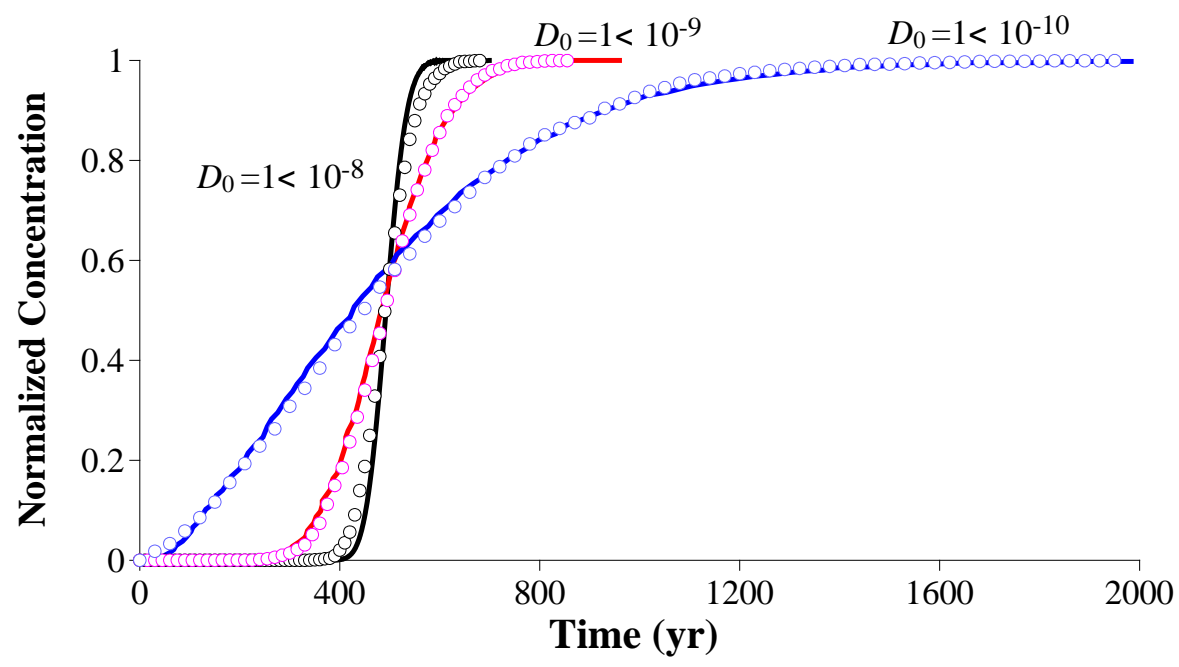

Figure 1. Numerical tests of RWHet3.25_NUFT.f90: Variable free-water diffusion coefficient $D_{0}$ - the simulated breakthrough curves in fracture continuum using RWHet3.25_NUFT.f90 (symbols) versus analytical solutions (lines). The unit for $D_{0}$ is meter $^{2} /$ second. The other model parameters are: velocity $V=1 \mathrm{~m} /$ day, fracture dispersivity $\alpha_{L}=0 \mathrm{~m}$, fracture aperture $2 b=2 \times 10^{-5} \mathrm{~m}$, fracture spacing $2 B=1 \mathrm{~m}$, matrix porosity $\theta_{m}=0.1$, matrix tortuosity $\tau=0.25$, fracture retardation coefficient $R_{f}=1$, matrix retardation coefficient $R_{m}=1$, and the travel distance is $36 \mathrm{~m}$.
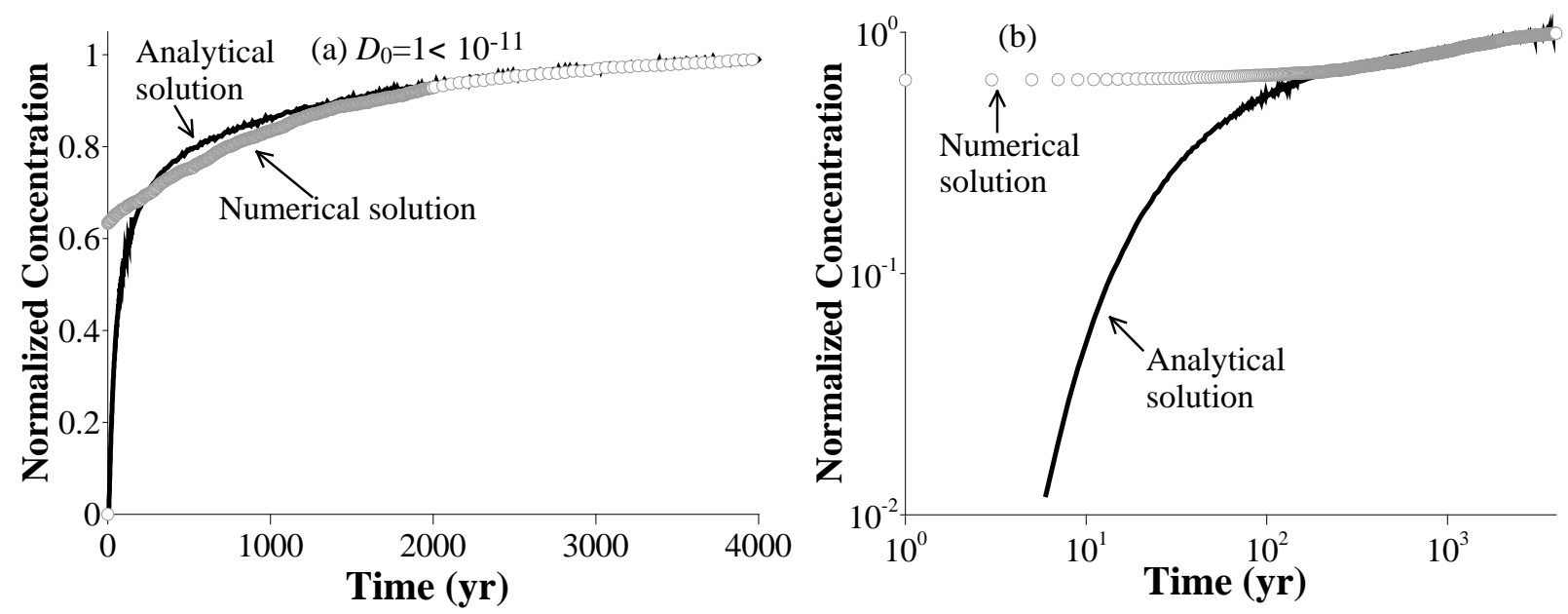

Figure 2. Numerical tests of RWHet3.25_NUFT.f90: A small free-water diffusion coefficient $D_{0}=1 \times 10^{-11}$ meter $^{2} /$ second - the simulated breakthrough curves in fracture continuum using RWHet3.25_NUFT.f90 (symbols) versus analytical solutions (lines). (b) is the log-log plot of (a). The other model parameters are the same as those shown in Figure 1. 


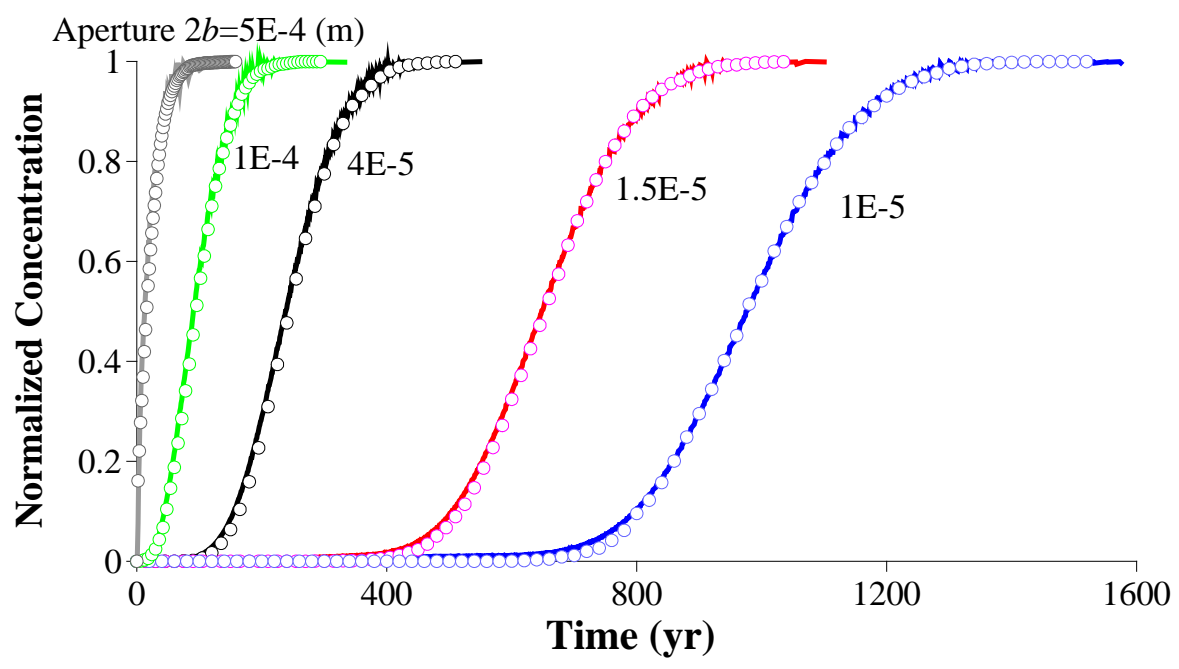

Figure 3. Numerical tests of RWHet3.25_NUFT.f90: Variable aperture $2 b$ (units: meter). The other model parameters are: (free-water) diffusion coefficient $D_{0}=1 \times 10^{-9} \mathrm{~m}^{2} / \mathrm{s}$, velocity $V=1 \mathrm{~m} /$ day, fracture dispersivity $\alpha_{L}=0 \mathrm{~m}$, fracture spacing $2 B=1 \mathrm{~m}$, matrix porosity $\theta_{m}=0.1$, matrix tortuosity $\tau=0.25$, fracture retardation coefficient $R_{f}=1$, matrix retardation coefficient $R_{m}=1$, and the travel distance is $36 \mathrm{~m}$.

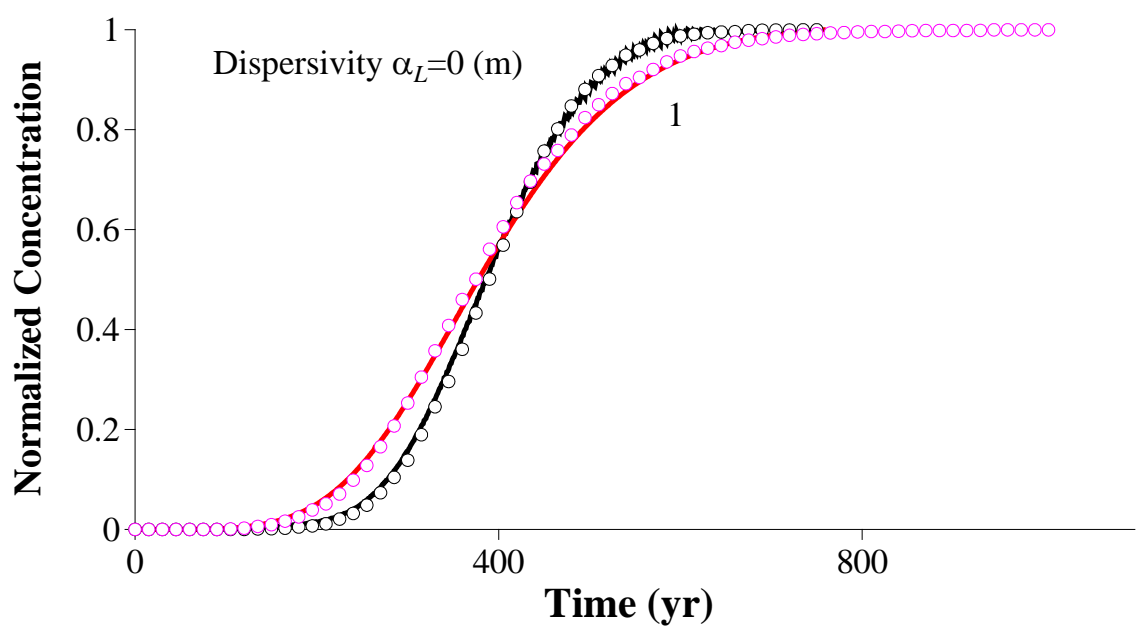

Figure 4. Numerical tests of RWHet3.25_NUFT.f90: Variable longitudinal dispersivity $\alpha_{\mathrm{L}}$ in fracture (units: meter). The other model parameters are: (free-water) diffusion coefficient $D_{0}=1 \times 10^{-9} \mathrm{~m}^{2} / \mathrm{s}$, velocity $V=1 \mathrm{~m} /$ day, fracture aperture $2 b=2.5 \times 10^{-5} \mathrm{~m}$, fracture spacing $2 B=1 \mathrm{~m}$, matrix porosity $\theta_{m}=0.1$, matrix tortuosity $\tau=0.25$, fracture retardation coefficient $R_{f}=1$, matrix retardation coefficient $R_{m}=1$, and the travel distance is $36 \mathrm{~m}$. 


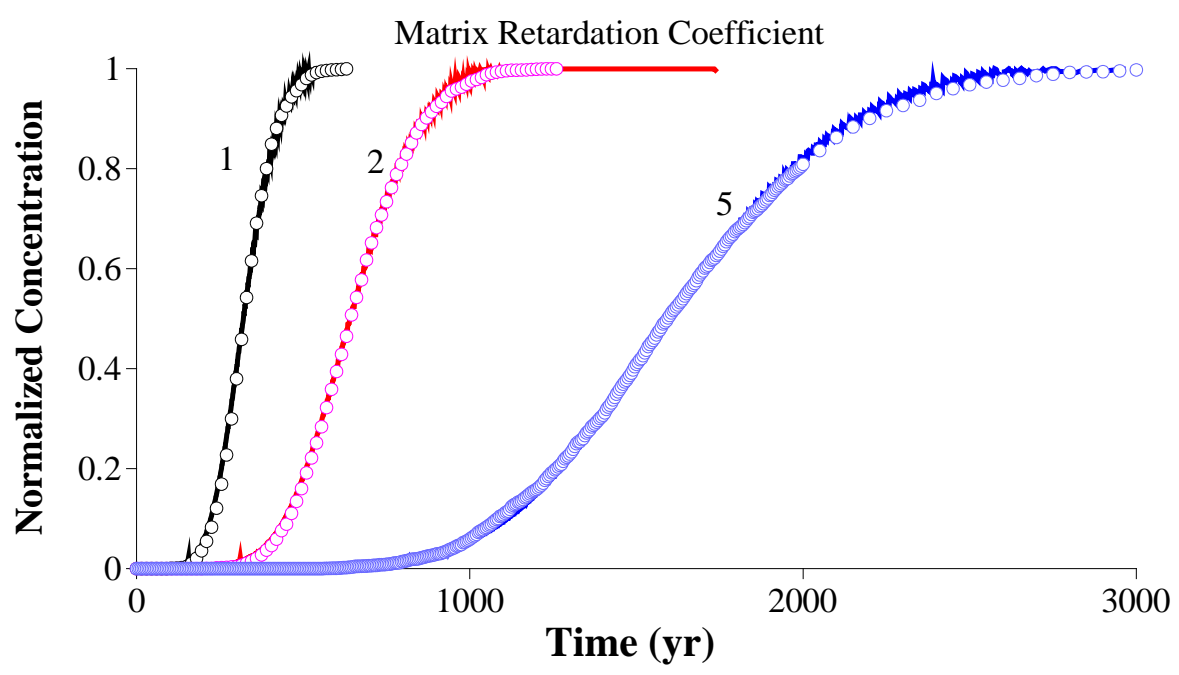

Figure 5. Numerical tests of RWHet3.25_NUFT.f90: Variable matrix retardation coefficient $R_{m}$. The other model parameters are: (free-water) diffusion coefficient $D_{0}=1 \times 10^{-9}$ $\mathrm{m}^{2} / \mathrm{s}$, velocity $V=1 \mathrm{~m} /$ day, fracture aperture $2 b=3 \times 10^{-5} \mathrm{~m}$, fracture spacing $2 B=1 \mathrm{~m}$, fracture dispersivity $\alpha_{L}=0 \mathrm{~m}$, matrix porosity $\theta_{m}=0.1$, matrix tortuosity $\tau=0.25$, fracture retardation coefficient $R_{f}=1$, and the travel distance is $36 \mathrm{~m}$.

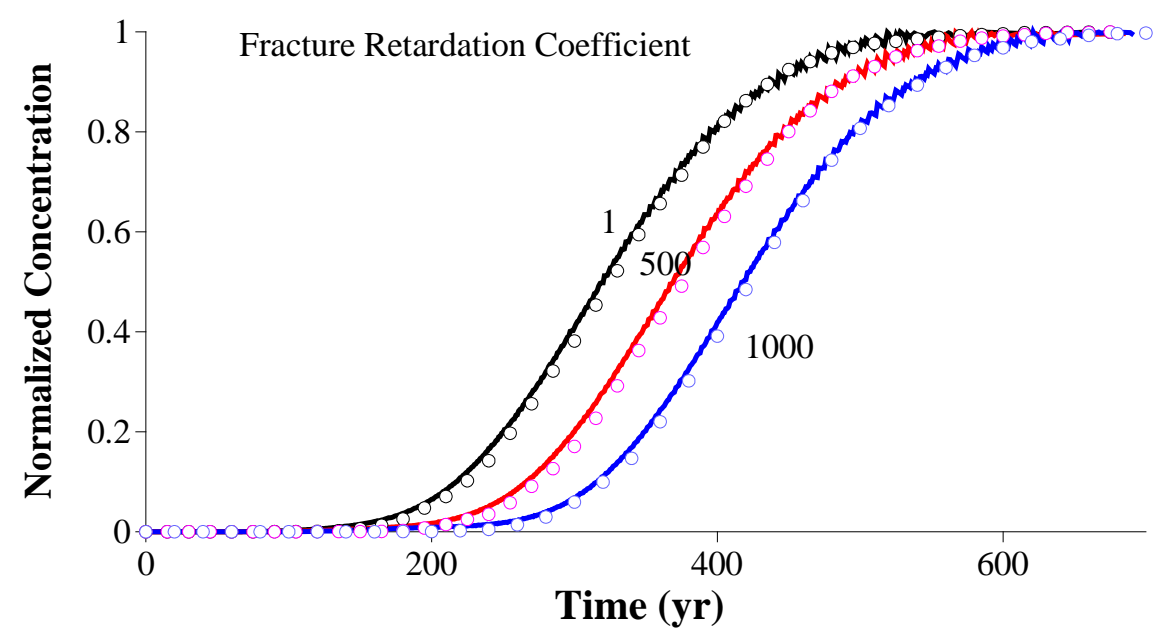

Figure 6. Numerical tests of RWHet3.25_NUFT.f90: Variable fracture retardation coefficient $R_{f}$. The other model parameters are: (free-water) diffusion coefficient $D_{0}=1 \times 10^{-9}$ $\mathrm{m}^{2} / \mathrm{s}$, velocity $V=1 \mathrm{~m} /$ day, fracture aperture $2 b=3 \times 10^{-5} \mathrm{~m}$, fracture spacing $2 B=1 \mathrm{~m}$, fracture dispersivity $\alpha_{L}=0 \mathrm{~m}$, matrix porosity $\theta_{m}=0.1$, matrix tortuosity $\tau=0.25$, matrix retardation coefficient $R_{m}=1$, and the travel distance is $36 \mathrm{~m}$. 

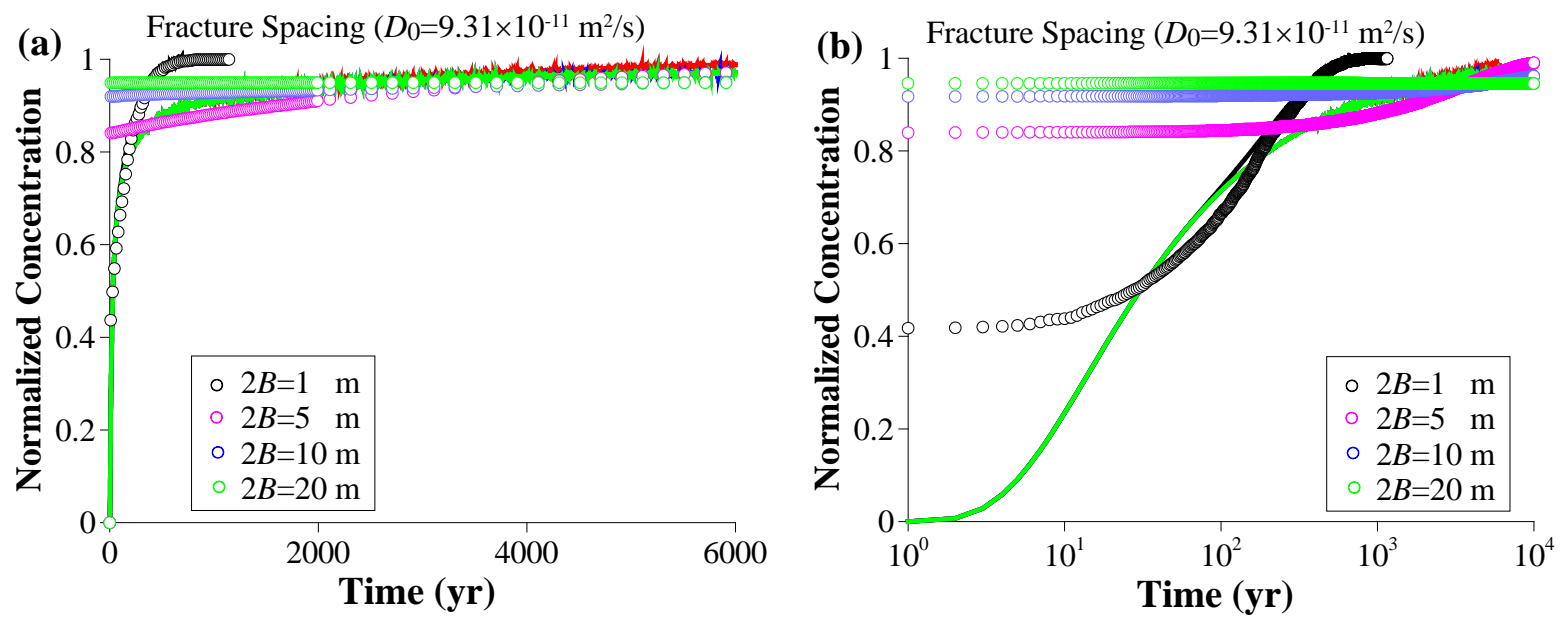

Figure 7. Numerical tests of RWHet3.25_NUFT.f90: Influence of fracture spacing on the BTC: analytical solutions (lines) versus particle-tracking results of RWHet3.25_NUFT.f90 (symbols). (b) is the semi-log plot of (a), to show the early portion of BTC. The unit for fracture spacing (actually $2 B$ ) is meters. The other model parameters are: (free-water) diffusion coefficient $D_{0}=9.31 \times 10^{-11} \mathrm{~m}^{2} /$ second, velocity $V=1 \mathrm{~m} /$ day, fracture dispersivity $\alpha_{L}=0.01 \mathrm{~m}$, fracture aperture $2 b=1 \times 10^{-4} \mathrm{~m}$, matrix porosity $\theta_{m}=0.1$, matrix tortuosity $\tau=0.25$, fracture retardation coefficient $R_{f}=1$, matrix retardation coefficient $R_{m}=1$, and the travel distance is $36 \mathrm{~m}$.

The particle tracking results generally match the analytical solutions for the freewater diffusion coefficient $D_{0}$ between $1 \times 10^{-8}$ and $1 \times 10^{-10} \mathrm{~m}^{2} / \mathrm{s}$ (Figure 1 ). Note that the effective diffusion coefficient used above is $D^{*}=D_{0} \tau \theta_{m}$ (following the definition by Liu et al. [2000], page 711). If a very small $D_{0}$ is used (such as $1 \times 10^{-11} \mathrm{~m}^{2} / \mathrm{s}$ ) (Figure 2), the particle tracking solution tends to overestimate the early breakthrough curve (BTC), similar to that observed by Hassan [2002]. Therefore, the transfer probability method proposed by Liu et al. [2000] is not applicable for small diffusion coefficients. Improvement of the particle tracking approach to account for this deficiency will be discussed in Section 3.

If $D_{0}$ is not extremely small, the particle tracking results match the analytical solutions for various fracture apertures (Figure 3). Acceptable matches between numerical and analytical solutions are also found for various dispersivities (Figure 4) and matrix retardation coefficients (Figure 5). The fracture retardation coefficient affects the dynamics of particle transport (Figure 6), by affecting not only the transport parameters (such as the effective velocity, dispersion coefficient, and fracture porosity), but also the transfer probability $P_{f m}$ (see Eq. (5)). The latter, however, has not been considered by previous studies.

Apparent discrepancy between the numerical and analytical solutions is found for a relatively large fracture spacing (Figure 7), which has also been identified by Pan and Bodvarsson [2002]. Such discrepancy leads to the correction discussed in Section 3. 


\subsection{Fracture/Matrix properties at the Rainer Mesa site}

Here we use parameters obtained for the Rainer Mesa (RM) site, Nevada, fracture/matrix to re-calculate the tracer BTCs for a generic radionuclide. A diffusion value for ${ }^{3} \mathrm{H}$ was selected, which has the largest free water diffusion coefficient. If the transfer probability method proposed by Liu et al. [2000] cannot capture the dynamics of ${ }^{3} \mathrm{H}$, then it can also be problematic in simulating the transport of other isotopes, such as ${ }^{241} \mathrm{Am}$ (the free-water diffusion coefficient $\left.D_{0}=3.06 \times 10^{-10} \mathrm{~m}^{2} / \mathrm{second}\right)$ and ${ }^{90} \mathrm{Sr}\left(D_{0}=\right.$ $1.29 \times 10^{-9} \mathrm{~m}^{2} /$ second). The model parameters for this generic radionuclide are: the freewater diffusion coefficient $D_{0}=9.31 \times 10^{-9} \mathrm{~m}^{2} /$ second, fracture retardation coefficient $R_{f}=$ 12.95 , matrix tortuosity $\tau=0.2$, matrix porosity $\theta_{m}=0.25$, matrix retardation coefficient $R_{m}=24192.9$, fracture aperture $(2 b)=5 \times 10^{-4}$ meter, and fracture spacing $(2 B)=10$ meter. Note that values of $R_{f}$ and $R_{m}$ are based on an average sorption coefficient $K_{d}$ for ${ }^{241} \mathrm{Am}$ in zeolitized volcanic tuffs.

The particle tracking result however is quite different from the analytical solution (Figure 8). RWHet3.25_NUFT.f90 overestimates the BTC at early times: almost all particles (2949 out of 2950 particles) exit the model domain at time $t \approx 4$ yrs. The remaining particle stays in the model until the end of the modeling period $(t=8,000 \mathrm{yrs})$. The analytical solution, however, shows that no particles should exit the model domain before $t=8,000$ yrs.

(a)

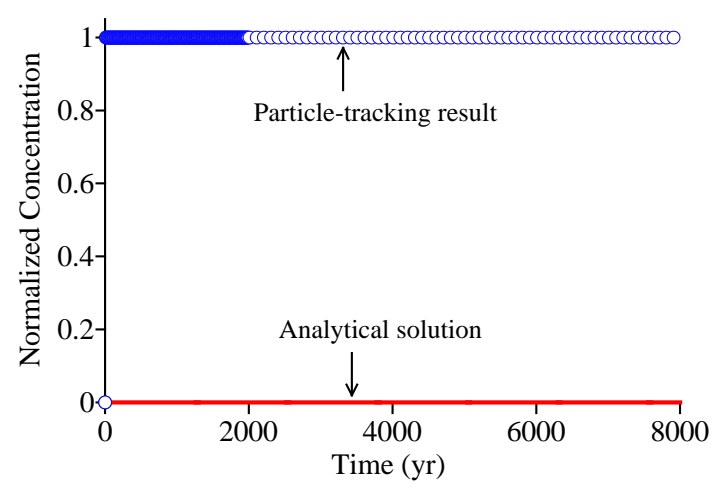

(b) Log-log plot of (a)

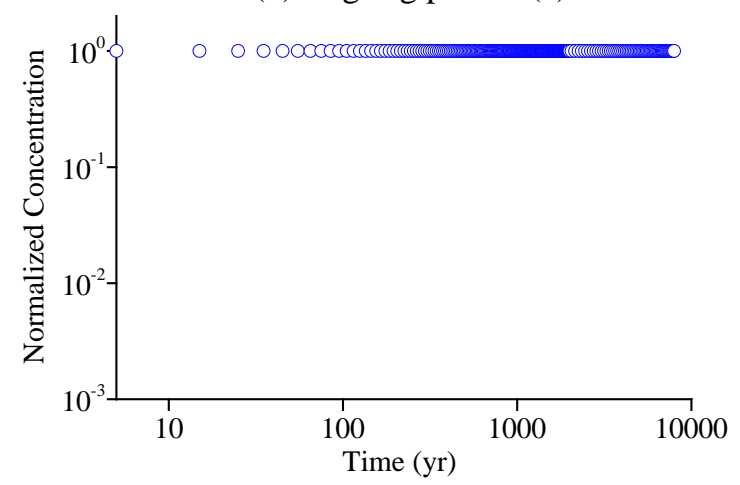

Figure 8. The simulated solution (blue symbols) versus the analytical solution (the red line) of tracer breakthrough curves of RWHet3.25_NUFT.f90 for RM site model parameters (see the text for the value of each parameter). In (a), $R_{m}=24192.9$. (b) is the log-log plot of (a).

The failure of RWHet3.25_NUFT.f90 with the Liu et al. [2000] may be due to the resultant small transfer probabilities (Table 1 ). The probability of a fracture particle to enter the matrix is as low as $3.2 \times 10^{-7}$, which is constant over the entire modeling period. In the numerical case we tested, only one particle could enter the matrix (note that all 2950 particles were at the fracture at the beginning of the simulation), and remained in the matrix for an extremely long time (due to the small transfer probability from matrix to 
fracture, which is $3.3 \times 10^{-14}$ ). For simplicity, we ignored dispersion within the fracture, and hence particles exited the model domain at the same time.

Table 1. Computed transfer probabilities for various matrix retardation coefficients.

\begin{tabular}{ccccc}
\hline Case & $\boldsymbol{R}_{\boldsymbol{m}}$ & $\boldsymbol{R}_{\boldsymbol{f}}$ & $P_{f m}$ & $P_{m f}$ \\
\hline $\mathbf{1}$ & 24192.9 & 12.95 & $3.2 \times 10^{-7}$ & $3.3 \times 10^{-14}$ \\
$\mathbf{2}$ & 5 & 12.95 & $1.5 \times 10^{-3}$ & $7.7 \times 10^{-7}$ \\
$\mathbf{3}$ & 2 & 12.95 & $3.8 \times 10^{-3}$ & $4.8 \times 10^{-6}$ \\
$\mathbf{4}$ & 1 & 12.95 & $7.6 \times 10^{-3}$ & $1.9 \times 10^{-5}$ \\
\hline
\end{tabular}

The small transfer probabilities are likely attributed to the large matrix retardation coefficient $R_{m}$. To explore the influence of $R_{m}$ on particle tracking in RWHet3.25_NUFT.f90, we tested three more cases with smaller $R_{m}$ (Table 1). The corresponding transfer probabilities increase with the decrease of $R_{m}$. Results (Figure 9) also show that the numerical solution approaches the analytical solution gradually.

The strong influence of $R_{m}$ on $P_{f m}$ and $P_{m f}$ can be seen from the transfer probability formulas (5) and (6). In both (5) and (6), the two factors $R_{m}$ and $S$ can be combined. In other words, the effect of increasing $R_{m}$ is functionally equivalent to increasing fracture spacing. The numerical examples in the above subsection show that the RWHet3.25_NUFT.f90 will fail for a large value of fracture spacing (Figure 7). Note that the increase of $R_{m}$ also decreases the diffusion in matrix, and hence delays the motion of particles (Figure 5).

Formulas (5) and (6) also show that the fracture retardation coefficient $R_{f}$ affects only the transfer probability from fracture to matrix $P_{f m}$. In (5), the two factors $R_{f}$ and $b$ can be combined, implying that the increase of $R_{f}$ can be functionally equivalent to the increasing of fracture aperture. The above numerical examples show that the particle tracking scheme is robust for a large range of fracture aperture (Figure 2), including the value found for the RM site. It also noteworthy that the increase of $R_{f}$ will also decrease the advection and dispersion for particles in fractures, resulting in a delayed BTC (see for example, Figure 6).

Therefore, the transfer probability method proposed by Liu et al. [2000] can capture the particle dynamics in fracture/matrix systems for most conditions, except for:

1) A small diffusion coefficient (i.e., the free-water diffusion coefficient $D_{0} \leq 1 \times 10^{-11}$ meter $^{2} /$ second);

2) Moderate fracture spacing (such as $2 B \geq 1$ meter);

3) Most values of retardation coefficient for matrix (i.e., $R_{m} \geq 2$ ). 

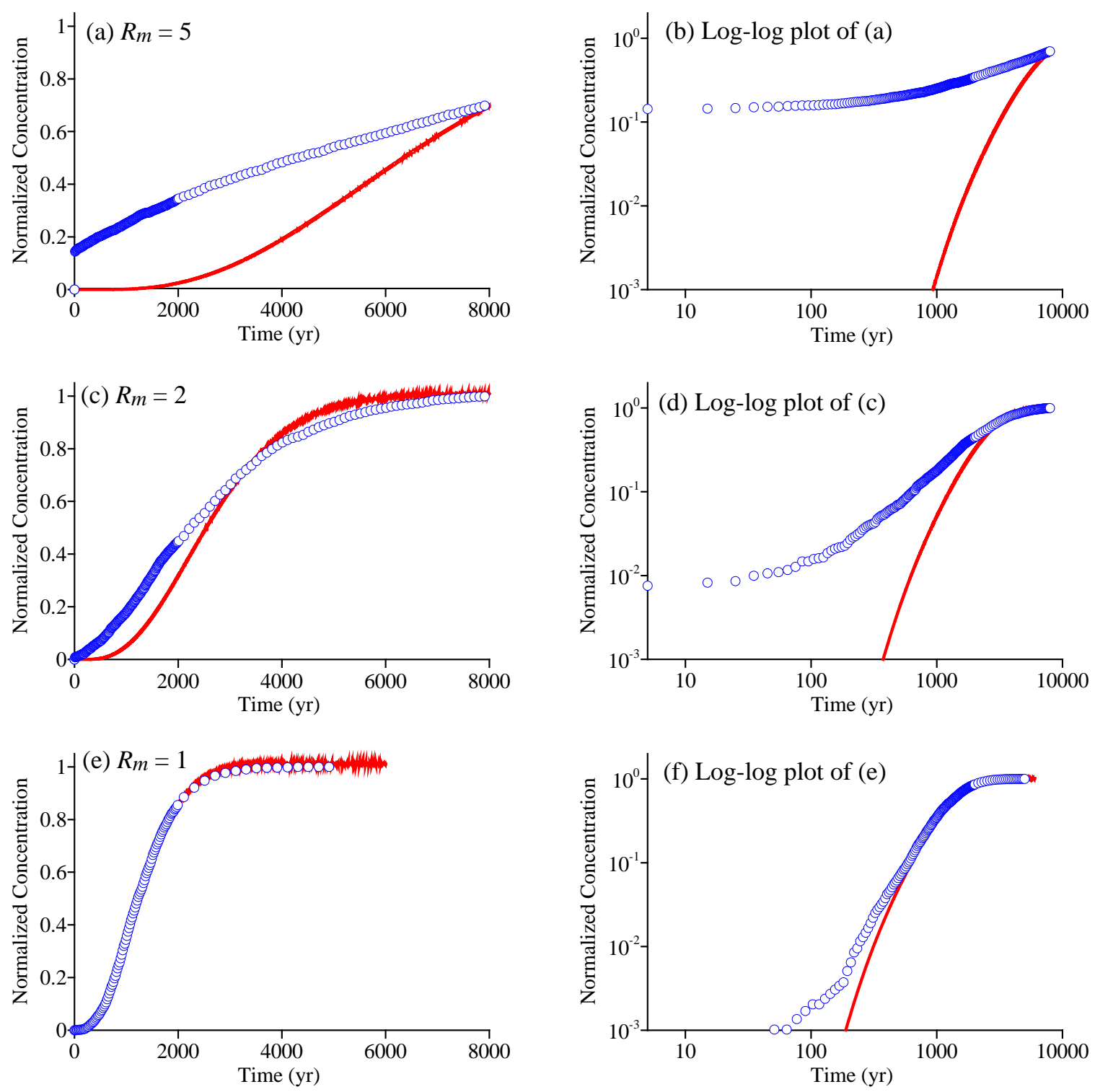

Figure 9. The simulated solution using RWHet3.25_NUFT.f90 (blue symbols) versus the analytical solution (the red line) of tracer breakthrough curves, where the model parameters are the same as Figure 7, except for the matrix retardation coefficient $R_{m}$. The right column is the log-log plot of the left column to show the tail at early times. 


\section{THE DIRECT NUMERICAL SIMULATION (DNS) OF CONTAMINANT TRANSPORT IN FRACTURED POROUS MEDIA: THE DNS-REFLECTIVE METHOD}

The DNS-Reflective method is expected to simulate solute transport throughout the entire parameter space. The core of the DNS-Reflective approach is to accurately and efficiently describe the particle dynamics across the interface between the fracture and matrix (Figure 10). Hence, it is a specific particle-tracking method for modeling solute transport in composite media where sharp contrasts exist between velocity, porosity, diffusion, dispersion and/or retardation. These are the same types of conditions that exist across a fracture-matrix interface. Previously developed particle tracking algorithms (e.g., LaBolle et al. [1996, 2000], LaBolle and Zhang [2006], and Bechtold et al. [2011]), several of which are already included in RWHet, can be applied to track particles across a fracture-matrix interface.

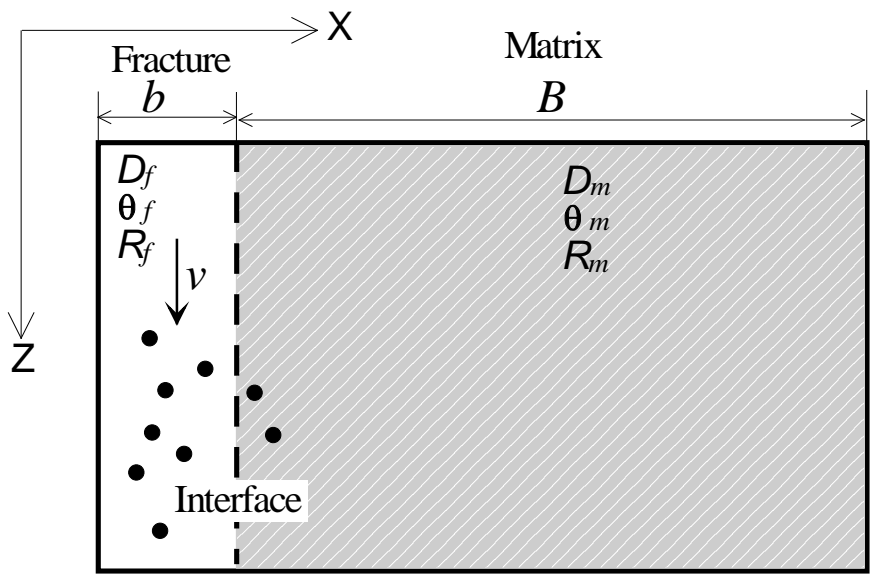

Figure 10. Conceptual model of a single fracture-matrix system. In the figure, $D$ denotes the molecular diffusion coefficient, $\theta$ denotes the porosity, and $R$ is the retardation coefficient. The suffix " $p$ " denotes fracture, and “ $m$ " denotes matrix. The dashed line denotes the discrete interface between the fracture and the matrix.

The DNS-Reflective approach contains the following three major steps:

Step 1 - Calculate the independent transport components for each particle.

Standard particle tracking schemes can be used to calculate the advective and dispersive displacement of each particle during each jumping event:

$$
\begin{aligned}
& x(t+d t)=x(t)+v(x) d t+w \sqrt{D_{x} d t} \\
& z(t+d t)=z(t)+w \sqrt{D_{z} d t}
\end{aligned}
$$

where $w$ is a uniform random number with mean zero and variance 1 . The core of this step is to define the appropriate time step $d t$ used in (8). To limit the jump size of a single particle during one step to be less than the (half) aperture $b, d t$ should be defined as: 


$$
d t \leq\left(\frac{b}{6}\right)^{2} \frac{1}{D_{0} / R_{f}} \quad,
$$

where $D_{0}$ is the free-water diffusion coefficient and $R_{f}$ is the fracture retardation coefficient.

If there is an advective flux from fracture to matrix, $d t$ should be smaller than (9), so that the particle moves at least twice before encountering the fracture/matrix interface. It is also noteworthy that additional transport components can be added conveniently to particle trajectories, including advection in the matrix.

Step 2 - Apply the one-side reflection scheme to capture the particle dynamics across the fracture/matrix interface.

The one-side reflection scheme proposed recently by Bechtold et al. [2011] is selected for this step. This scheme corrects the standard reflection method by splitting the time step nonlinearly for particles across the discrete interface. It also increases significantly the computational efficiency by transforming the reflection barrier method from a two-side into a one-side reflection scheme.

Note that in the one-side reflection scheme, the particle can jump "freely" from matrix to fracture, due to the one-side reflection probability defined below:

$$
\begin{aligned}
& P_{f \rightarrow m}=1-\frac{\theta_{m} \sqrt{D_{m} / R_{m}}}{\theta_{f} \sqrt{D_{f} / R_{f}}}, \\
& P_{m \rightarrow f}=0
\end{aligned}
$$

if

$$
\theta_{f} \sqrt{D_{f} / R_{f}}>\theta_{m} \sqrt{D_{m} / R_{m}}
$$

Here $P_{f \rightarrow m}$ is the reflection probability from fracture to matrix, and $P_{m \rightarrow f}$ is the reflection probability from matrix to fracture (note that a zero reflection probability means that no particles can be reflected at the interface, or in other words, each particle can move "freely" if it starts in matrix). When the two retardation coefficients $R_{m}$ and $R_{f}$ equal 1, (10) reduces to the formula proposed by Bechtold et al. [2011]. A uniform [0 1] random number $U$ is generated and compared to the above probabilities. If the particle is located in the fracture and $U>P_{f \rightarrow m}$, then the particle can cross the fracture/matrix interface during the current time step. When the particle is located in the matrix, it can move freely in either direction. This scheme is coded and verified, with some examples shown in Figure 11. 

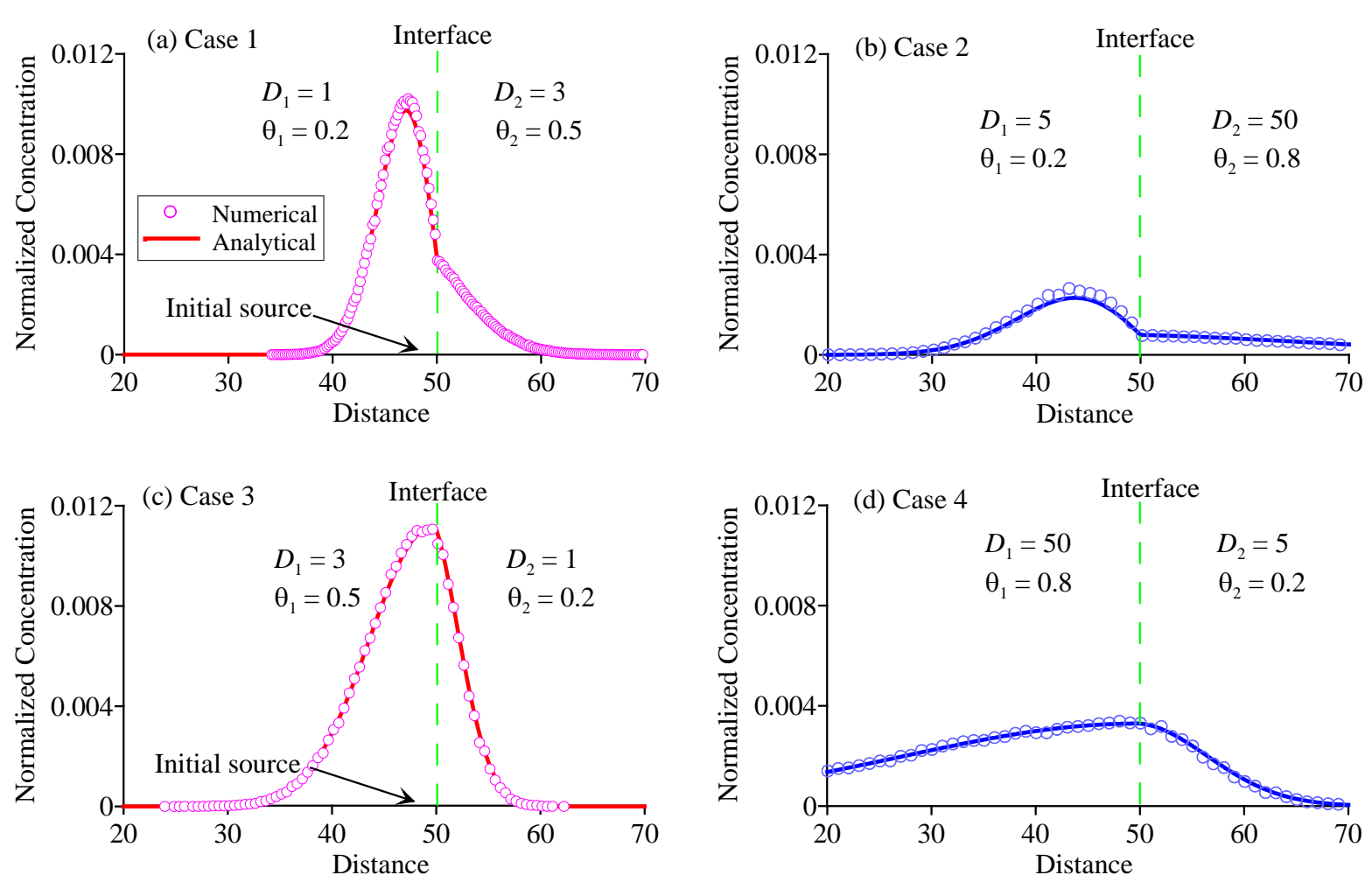

Figure 11. DNS-Reflective method: Numerical examples of the one-side reflection scheme: the simulated results (symbols) versus the analytical solute concentration distributions (lines, from Carslaw and Jaeger [1959]) at $t=5$ for a composite media with discrete diffusion coefficients and effective porosity. The vertical green line indicates the location of the "reflective" boundary. An instantaneous point source is located at $x=48$. For simplicity, $D$ shown in the figure is dimensionless (note the purpose of this experiment is to explore the applicability of the Lagrangian scheme to capture particle dynamics across an abrupt interface).

Step 3 - Split the time step $d t$ for each particle spent in the fracture and the matrix domain, and then repeat the above steps until reaching the final simulation time.

There are two loop cycles of the above 3-step scheme. The outer iteration is for time, and the inner iteration is for the number (i.e., sequence) of particles.

We systematically test the above 3-step DNS-Reflective method. Results (Figure 12) show that the DNS-Reflective solutions generally match the analytical solutions to a single fracture within an infinite matrix [Tang et al., 1981], if the time step $d t$ is properly defined. For example, for case 6 shown by Table 2 and Figure 12(f), Eq. (9) shows that $d t \leq 50.2$ day. In the DNS-Reflective simulations, we test three time steps: $1 \times 10^{-2}$ day, $1 \times 10^{-3}$ day, and $1 \times 10^{-4}$ day. Because the three time steps are much less than 50.2 day, the three simulated breakthrough curves are almost identical to the analytical solutions.

For case 2, the minimum time step defined by Eq. (9) is $d t \leq 5.02 \times 10^{-5}$ day (Table 2). In the four time steps tested in the DNS-Reflective simulations (Figure 12(b)), 
only the smallest one (i.e., $2.5 \times 10^{-5}$ day) meets the criterion of Eq. (9) and hence it can generate the similar result as the analytical solution.

Left: different aperture $b$
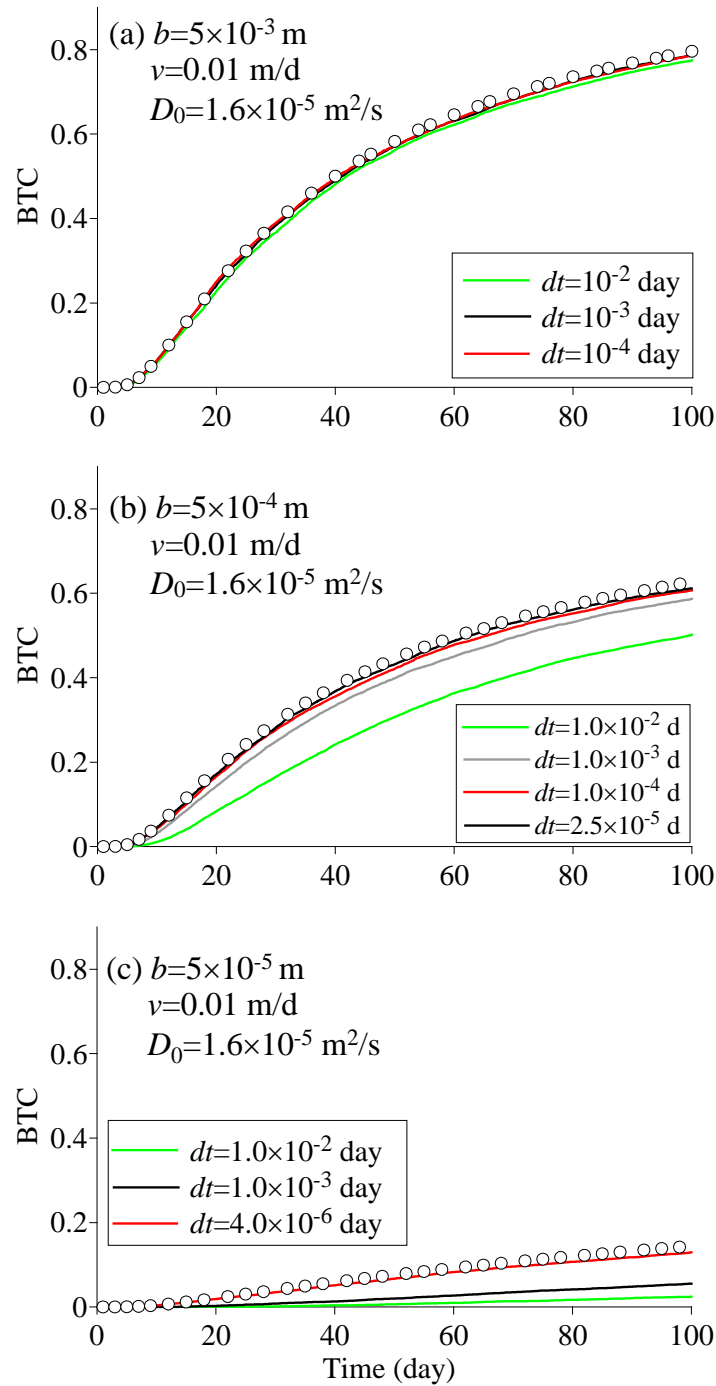

Right: different diffusion coefficient $D_{0}$
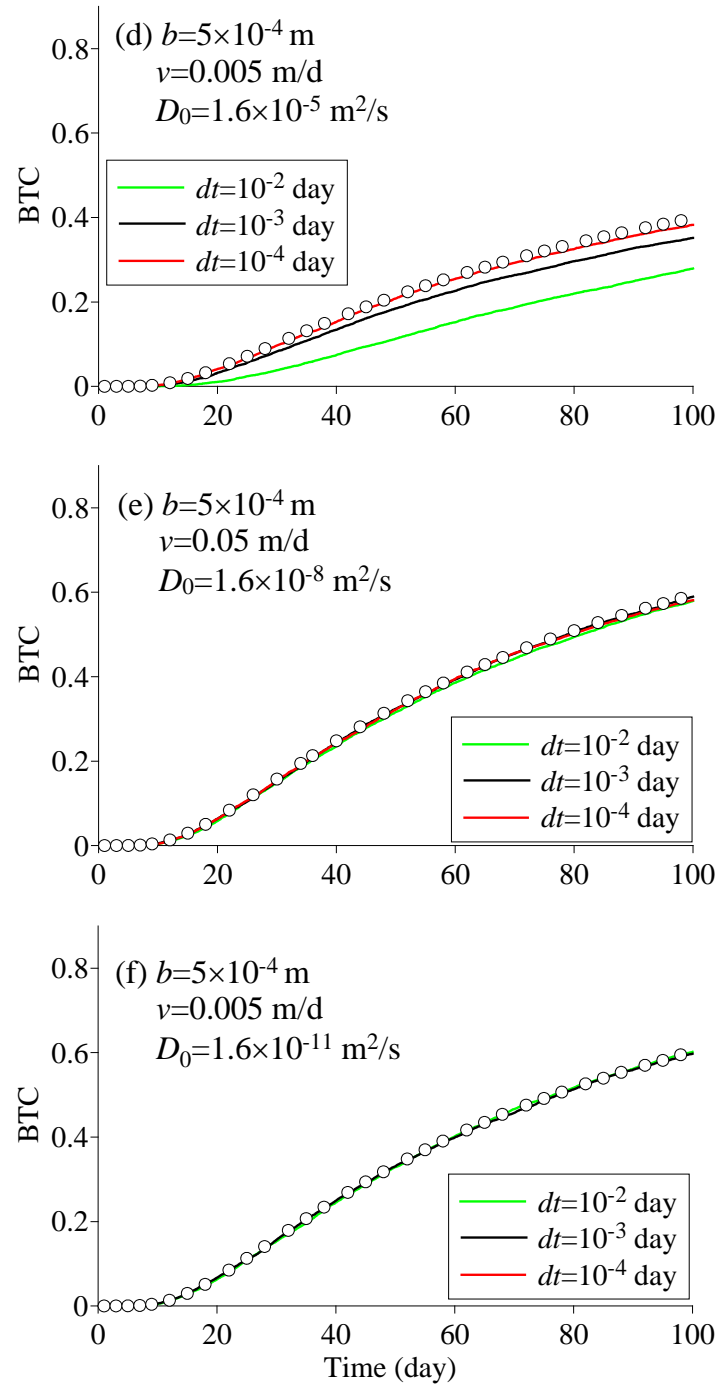

Figure 12. DNS-Reflective method: Influence of (half) aperture $b$ (left figures) and the freewater diffusion coefficient $D_{0}$ (right figures) on the breakthrough curve for the fracture at depth $z=0.666 \mathrm{~m}$ : the DNS-Reflective solutions (lines) versus the analytical solutions (symbols, Tang et al. [1981]). The (half) aperture is $b=5 \times 10^{-3}$ $\mathrm{m}(\mathrm{a}), 5 \times 10^{-4} \mathrm{~m}$ (b) (Note that the fracture aperture at Rainier Mesa is $2 b=5 \times 10^{-4}$ $\mathrm{m}$ ), and $5 \times 10^{-5} \mathrm{~m}(\mathrm{c})$, respectively. $D_{0}$ is $1.6 \times 10^{-5} \mathrm{~m}^{2} / \mathrm{s}(\mathrm{d}), 1.6 \times 10^{-8} \mathrm{~m}^{2} / \mathrm{s}(\mathrm{e})$, and $1.6 \times 10^{-11} \mathrm{~m}^{2} / \mathrm{s}(\mathrm{f})$, respectively. At Rainier Mesa, the range of free water diffusion coefficient is from $3.06 \times 10^{-10} \mathrm{~m}^{2} / \mathrm{s}$ (for isotope ${ }^{241} \mathrm{Am}$ ) to $9.31 \times 10^{-9} \mathrm{~m}^{2} / \mathrm{s}$ (for isotope $\left.{ }^{3} \mathrm{H}\right)$. In the legend, " $d t$ " denotes the time step used in the particle tracking. The other model parameters are: matrix porosity $\theta_{m}=0.01$, tortuosity $\tau=0.1$, matrix retardation coefficient $R_{m}=1$, fracture retardation coefficient $R_{f}=1$, and the longitudinal dispersivity in fracture $\alpha_{\mathrm{L}}=0.5 \mathrm{~m}$ (the transverse dispersivity is 0 ). In all cases, 10,000 particles are released at the beginning of the simulation. 
Table 2. The six numerical cases shown in Figure 12 and the time step for particle tracking. In the legend, $T_{1}=(b / 6)^{2} / D_{0}$, and $T_{2}=(b / 4)^{2} / D_{0}$.

\begin{tabular}{clclll}
\hline Case & Figure & Aperture $\boldsymbol{b}(\mathbf{m})$ & $\boldsymbol{D}^{\boldsymbol{*}}\left(\mathbf{m}^{\mathbf{2}} / \mathbf{s}\right)$ & $\boldsymbol{T}_{\mathbf{1}}(\mathbf{d a y})$ & $\boldsymbol{T}_{\mathbf{2}}$ (day) \\
\hline $\mathbf{1}$ & Figure 2(a) & $5 \times 10^{-3}$ & $1.6 \times 10^{-5}$ & $5.02 \times 10^{-3}$ & $1.13 \times 10^{-2}$ \\
$\mathbf{2}$ & Figure 2(b) & $5 \times 10^{-4}$ & $1.6 \times 10^{-5}$ & $5.02 \times 10^{-5}$ & $1.13 \times 10^{-4}$ \\
$\mathbf{3}$ & Figure 2(c) & $5 \times 10^{-5}$ & $1.6 \times 10^{-5}$ & $5.02 \times 10^{-7}$ & $1.13 \times 10^{-6}$ \\
$\mathbf{4}$ & Figure 2(d) & $5 \times 10^{-4}$ & $1.6 \times 10^{-5}$ & $5.02 \times 10^{-5}$ & $1.13 \times 10^{-4}$ \\
$\mathbf{5}$ & Figure 2(e) & $5 \times 10^{-4}$ & $1.6 \times 10^{-8}$ & $5.02 \times 10^{-2}$ & $1.13 \times 10^{-1}$ \\
$\mathbf{6}$ & Figure 2(f) & $5 \times 10^{-4}$ & $1.6 \times 10^{-11}$ & $5.02 \times 10^{1}$ & $1.13 \times 10^{2}$ \\
\hline
\end{tabular}

A single snapshot of particle positions at a specific time is also shown in Figure 13. Advective transport in the fracture is downward with particle transfer across the fracture/matrix interface, which is shown by the red, vertical dashed line in Figure 13. Obviously, if the fracture aperture is small, the particles inside the fracture cannot make a large horizontal jump during one step of motion. This limits the computational efficiency of the numerical method.

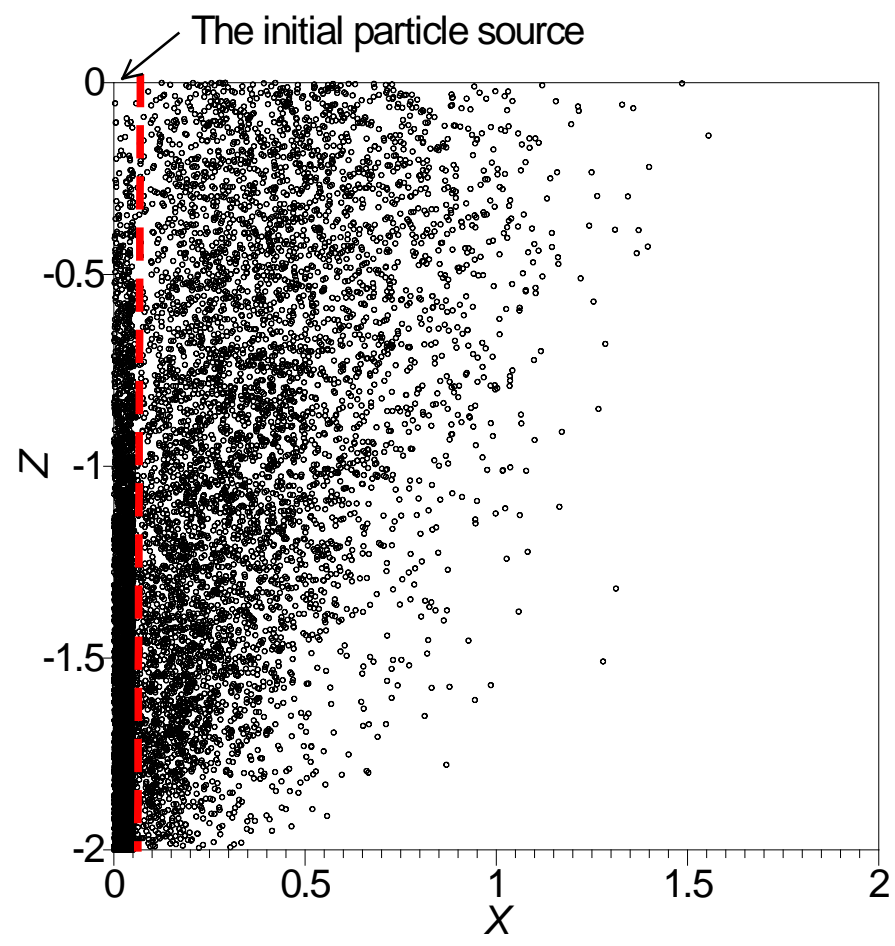

Figure 13. A snapshot (particle clouds) simulated by the DNS-Reflective method in a single fracture with downward transport and fracture-matrix particle transfer. 
In addition, we found that numerical experiments also imply that the minimum time step defined by (9) can be increased slightly by:

$$
d t \leq\left(\frac{b}{4}\right)^{2} \frac{1}{D^{*} / R_{f}}
$$

Numerical solutions generated by the minimum time step defined by (9) are still close to the analytical solutions.

To draw an intermediate conclusion, the efficiency and accuracy of the DNSReflective approach depends on the time step and the number of particles. A smaller diffusion coefficient results in a larger time step $d t$, and therefore, the DNS-Reflective method can be more computationally efficient. This behavior makes the method superior to the transfer probability approach discussed above. As shown in Figure 12 (e) and (f), the DNS-Reflective method is efficient since the time step $d t>10^{-3}$ day for all isotopes at the Rainier Mesa site (such as ${ }^{241} \mathrm{Am},{ }^{90} \mathrm{Sr},{ }^{3} \mathrm{H}$ ).

On the other hand, the DNS-Reflective method is time consuming for a small fracture aperture, since the time step size must be small enough for particles to jump at least once within the fracture. In addition, if the free-water diffusion coefficient $D_{0}$ is large, the DNS-Reflective method is less computationally efficient because a smaller time step $d t$ is also needed.

To increase the computational efficiency of the particle-tracking approach, the time step should be increased. To release the limitation of the small time step for the case of a small fracture-spacing and a large free-water diffusion coefficient, we will apply the DNS-Roubinet method in the next section.

\section{THE DNS-ROUBINET METHOD}

The core of Roubinet et al. 's [2009] method is the use of first-passage time distributions to compute the time each particle spends in the matrix, which leads to time steps considerably greater than the DNS-Reflective method. This should increase the computational efficiency of the DNS method significantly. In the following we briefly introduce the DNS-Roubinet method and the treatment in the programming.

The total time for a random-walking particle spent in a fracture/matrix system $t_{f m}$ with an infinite matrix is given in terms of the time in the fracture $t_{f}$ as

$$
P\left(t_{f m}<T\right)=\operatorname{erf}\left(\frac{t_{f} \theta_{m} \sqrt{D_{m}}}{b \sqrt{T-t_{f}}}\right),
$$

where "erf" denotes the error function; $T$ is the reference time; $\theta_{m}$ and $D_{m}$ represent the porosity and dispersion coefficient in the matrix, respectively (the same as Eq. (10)); and $b$ is the (half) fracture aperture (the same as above). Note here the time spent in the fracture, $t_{f}$, can be pre-assigned as the time step.

The inverse in terms of a uniform random deviate yields the following formula, using (13): 


$$
t_{f m}=t_{f}+\left[\frac{\mathrm{berf}^{-1}(U)}{t_{f} \theta_{m} \sqrt{D_{m}}}\right]^{-2},
$$

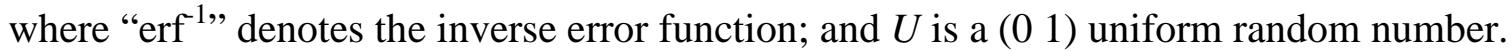
The time in the matrix is therefore $t_{m}=t_{f m}-t_{f}$.

In a system of parallel fractures, the first passage time from fracture to fracture is given as

$$
P\left(t_{2 S} \leq T\right)=2 P\left(X_{T} \geq 2 S\right)=\operatorname{erf}\left(\frac{\mathrm{S}}{\sqrt{\mathrm{D}_{\mathrm{m}} \mathrm{T}}}\right) \quad .
$$

Solving for $T$ yields:

$$
t_{2 S}=\left[\frac{\sqrt{D_{\mathrm{m}}} \operatorname{erf}^{-1}(U)}{S}\right]^{-2} .
$$

In this system, $t_{f m}=t_{f}+\min \left(t_{m}, t_{2 S}\right) ; t_{m}=t_{f m}-t_{f}$.

The particle-tracking numerical algorithm is:

(1) Loop over time and particles;

(2) Compute the advective travel time $t_{f}$ in a cell (note this value can be pre-defined);

(3) Compute the diffusion time $t_{\text {diff }}$ based on the analytical solution given by Tang et al. [1981], where the surrounding matrix is taken into account;

(4) Compute the first passage time $t_{2 S}$, which is the time for the particle to reach the next fracture.

(5) If $t_{\text {diff }}>t_{2 S}$, truncate $t_{\text {diff }}$ to $t_{2 S}$ (i.e., if the particle reaches another fracture then it did not need to diffuse as long); otherwise keep $t_{\text {diff }}$ unchanged;

(6) Sum times;

(7) End loops when all particles exit the system.

At the end the simulation, report the total times for all particles. These can be binned to yield a breakthrough curve.

The DNS-Roubinet method has been tested extensively against analytical solutions developed by Tang et al. [1981] and Sudicky and Frind [1982]. A few numerical tests are shown below (Figures 14 through 16). Figure 14 shows that the DNSRoubinet method works well for a wide range of fracture aperture and diffusion coefficient, for a single fracture system.

The DNS-Roubinet method has its own disadvantage: For the parallel fracture system, the truncation of first passage time distribution creates apparent errors when the fracture spacing is small. Hence the DNS method works very well for the single fracture system, but it tends to erroneously predict BTCs for the parallel fracture system. 

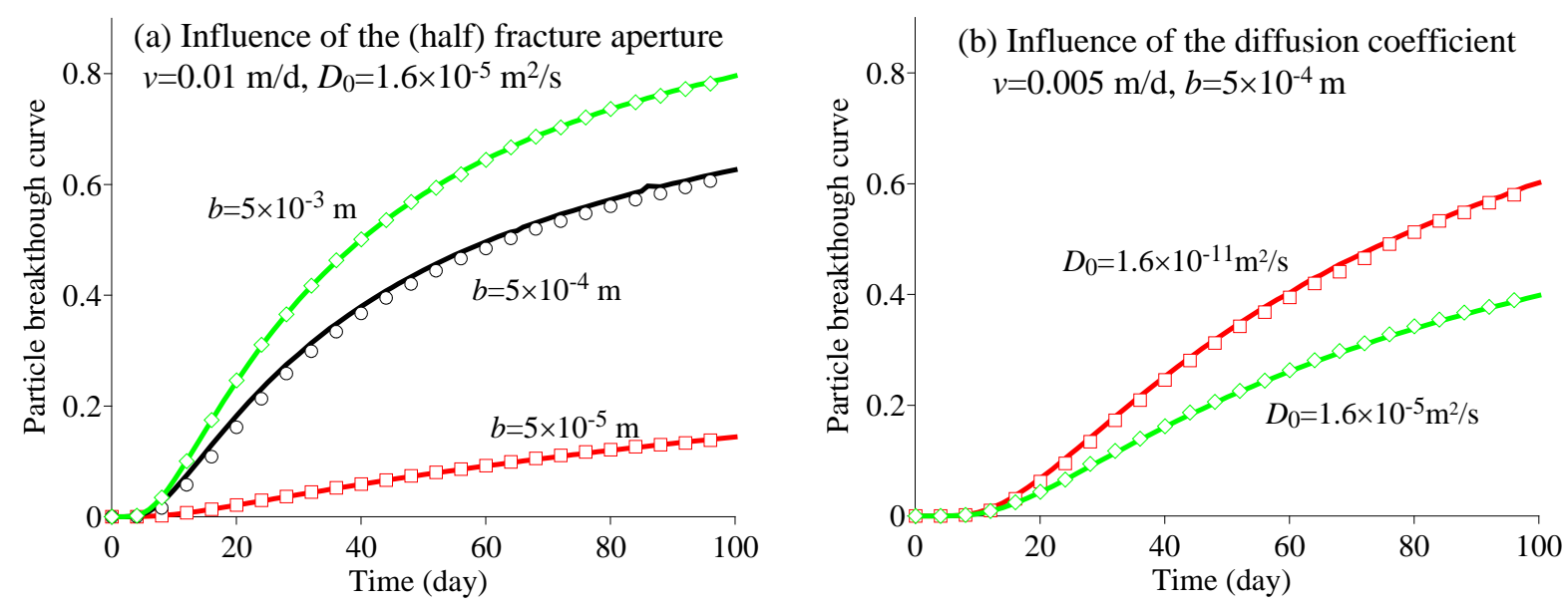

Figure 14. DNS-Roubinet solutions (symbols) versus the analytical solutions (lines) [Tang et $a l ., 1981]$ for a single fracture with variable fracture aperture $b(a)$ and diffusion coefficient $D_{0}$ (b).

Figure 15 shows that, similar to what we found in Figure 14, here the DNSRoubinet method works well for a single fracture system at various travel distances $L$ (along the fracture).

Figure 16 shows that the DNS-Roubinet method underestimates the analytic breakthrough at early times, and overestimates the breakthrough at late times, for the parallel fracture system.

Therefore, the DNS-Roubinet method is computationally more efficient than the DNS-Reflective method. It matches the analytical solution for the whole range of dispersion coefficients and fracture aperture for a single fracture system.

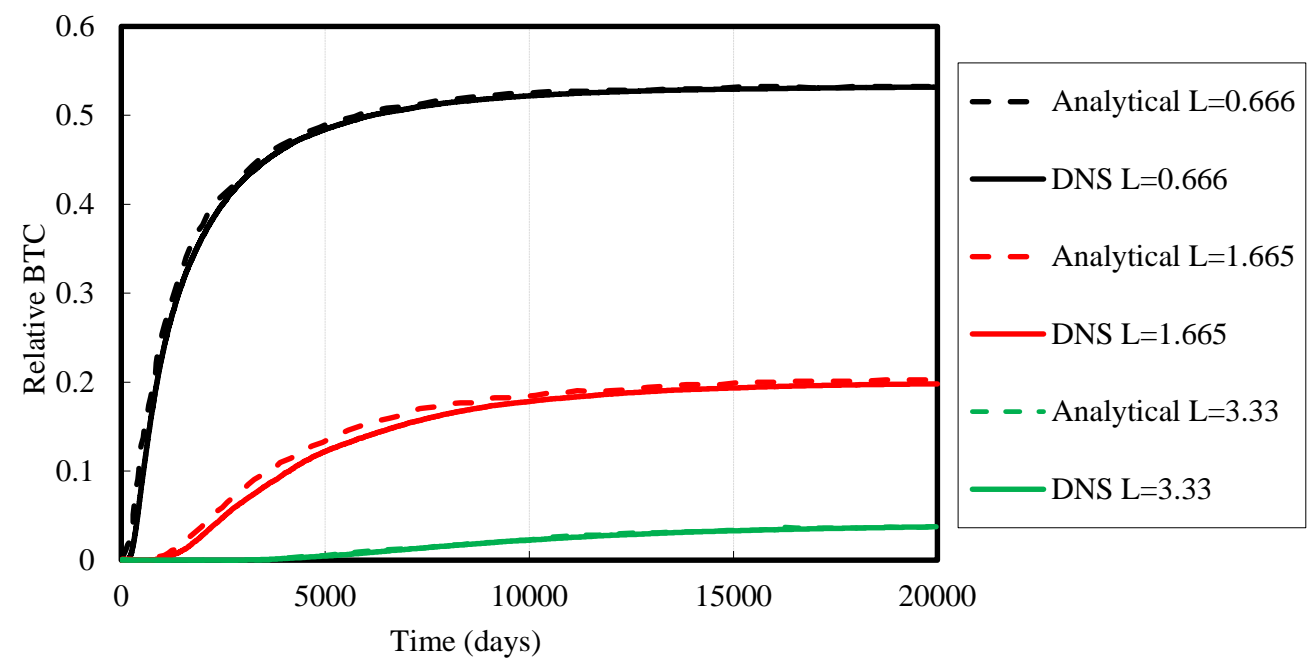

Figure 15. DNS-Roubinet solutions of the tracer breakthrough curves (symbols) versus the analytical solutions (lines) [Tang et al., 1981] for single fracture at various depths (denoted as "L" in the legend) in the fracture. See also Figure 5 in Tang et al. [1981]. 


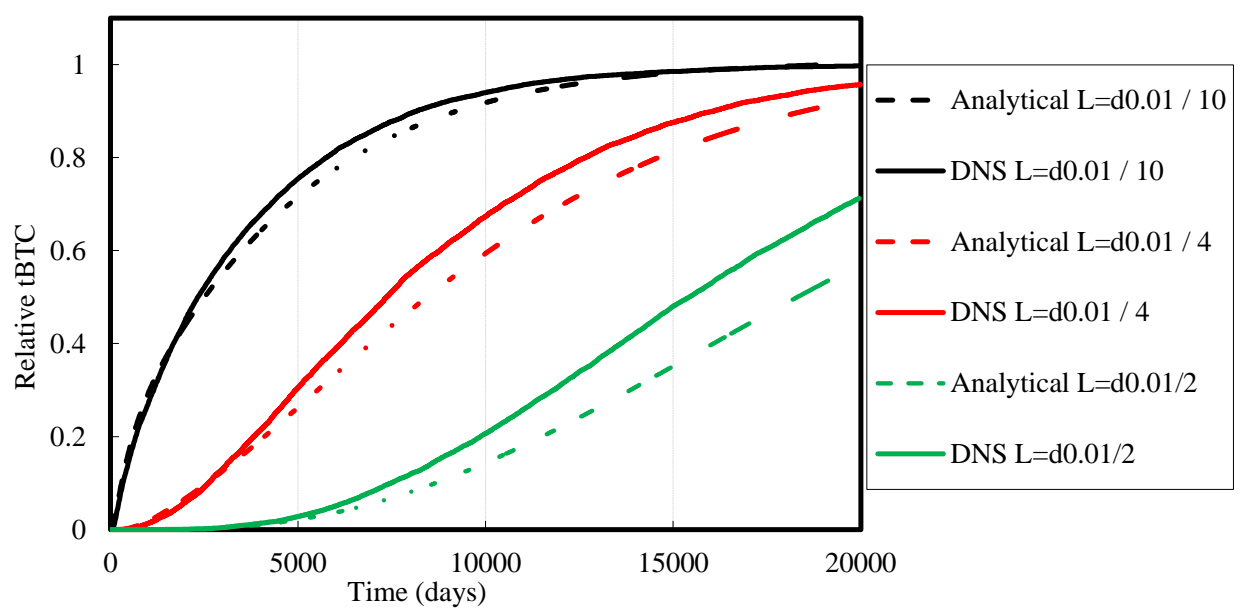

Figure 16. DNS-Roubinet solutions of the tracer breakthrough curves (symbols) versus the analytical solutions (lines) [Sudicky and Frind, 1982] for parallel fracture at various depths in the fracture. See also Figure 7 in Sudicky and Frind [1982].

\section{THE TRANSIENT RANGE APPROACH PROPOSED BY PAN AND BODVARSSON [2002]}

When the effective diffusion coefficient $D^{*}$ is small, the fracture spacing $B$ is large, and/or the matrix retardation coefficient is large, the transfer probability from fracture to matrix (defined by (5)) can be very small, underestimating the mass of particles entering into the matrix at early simulation times. This results in the overestimation of solute mass in fractures and erroneous early breakthroughs (Figure 7). Hence the modification of (5) is needed. The transient activity range approach developed by Pan and Bodvarsson [2002] is intended to solve this problem.

\subsection{Methodology of Pan and Bodvarsson [2002]}

The concept of “transient activity range” proposed by Pan and Bodvarsson [2002] can capture the dynamic feature of the particle transfer probability. The transfer probabilities are defined as [see Eq. (1) in Pan and Bodvarsson, 2002]

$$
\begin{gathered}
P_{f m}=\frac{F_{f m}}{Q_{f}+F_{f m}}\left[1-\exp \left(-\Delta t / \tau_{f}\right)\right], \\
P_{m f}=\frac{F_{m f}}{Q_{m}+F_{m f}}\left[1-\exp \left(-\Delta t / \tau_{m}\right)\right],
\end{gathered}
$$

where $F$ and $Q$ describe the strength of advection-dispersion process through the fracturematrix interface and the interfaces to adjacent grid cells in the same continuum, respectively (the subscripts show the direction of the flux) [Pan and Bodvarsson, 2002]:

$$
\begin{aligned}
& F_{f m}=\max \left(q_{f m} A_{f m}, 0\right)+\frac{D_{m} A_{f m}}{S_{f m}\left(t_{p}\right)}, \\
& F_{m f}=\max \left(-q_{f m} A_{f m}, 0\right)+\frac{D_{m} A_{f m}}{S_{f m}\left(t_{p}\right)},
\end{aligned}
$$




$$
\begin{aligned}
Q_{f} & =\sum_{i=1}^{N}\left[\max \left(q_{f} A_{i}, 0\right)+\frac{D_{f i} A_{i}}{S_{i}}\right], \\
Q_{m} & =\sum_{i=1}^{N}\left[\max \left(q_{m} A_{i}, 0\right)+\frac{D_{m i} A_{i}}{S_{i}}\right] .
\end{aligned}
$$

Parameters $A_{f m}$ and $A_{i}$ in (18) are the area of the fracture-matrix interface within the grid and the interface area to the $i$-th adjacent grid, respectively. Parameters $q_{f m}$ and $q_{i}$ in (18) are the water flux through the fracture-matrix interface within the grid and the interface to the $i$-th adjacent grid, respectively. Parameters $D_{m}, D_{f i}$ and $D_{m i}$ in (18) are effective dispersion coefficient of the matrix, the fracture continuum at the interface to the $i$-th adjacent grid, and the matrix continuum at the interface to the $i$-th adjacent grid, respectively. $S_{i}$ is the distance between the center of the cell and the $i$-th adjacent cell, and $S_{f m}\left(t_{p}\right)$ is the effective characteristic distance of the fracture-matrix system. In addition, the parameters $\tau_{f}$ and $\tau_{m}$ in (17) denote the characteristic times of the fracture and matrix continuum, respectively [Pan and Bodvarsson, 2002]:

$$
\begin{gathered}
\tau_{f}=\frac{V_{f} R_{f}}{F_{f m}+Q_{f}}, \\
\tau_{m}=\frac{V_{m}\left(t_{p}\right) R_{m}}{F_{m f}+Q_{m}},
\end{gathered}
$$

where $V$ and $R$ denote the volume of water and the retardation factor, respectively, for fractures and matrix.

In the above definitions, two variables vary with time, including the matrix volume $V_{m}\left(t_{p}\right)$ and the effective characteristic distance $S_{f m}\left(t_{p}\right)$ [Pan and Bodvarsson, 2002]:

$$
\begin{gathered}
V_{m}\left(t_{p}\right)=V_{m} \frac{B^{*}\left(t_{p}\right)}{B}, \\
S_{f m}\left(t_{p}\right)=S_{f m} \frac{B^{*}\left(t_{p}\right)}{B},
\end{gathered}
$$

where $S_{f m}$ denotes the characteristic length of the fracture-matrix system (e.g., 1/6 of the fracture spacing for a parallel fracture system as suggested by Liu et al. [2000]); and $t_{p}$ is the time elapsed since a pulse injected into the fractures (e.g., the age of each particle, if the instantaneous injection of particle source is at time zero). $B^{*}\left(t_{p}\right)$ is the activity range $\left(0 \leq B^{*}\left(t_{p}\right) \leq B\right)$ [Pan and Bodvarsson, 2002]:

$$
B^{*}\left(t_{p}\right)=\min \left(4 \sqrt{4 D_{m} t_{p} / R_{m}}, \quad B\right) \text {. }
$$




\subsection{Numerical examples of RWHet-Pan\&Bodvarsson}

The transient activity range approach proposed by Pan and Bodvarsson [2002] was implemented into RWHet. The updated RWHet is named "RWHetPan\&Bodvarsson". We tested this code extensively. The numerical results can be compared to the code DCPTV2.0 [Pan, 2002] where the transient activity range approach was originally developed.

The following section text describes five of these tests.

5.2.1. Test $1:{ }^{3} \mathrm{H}$ with variable free-water diffusion coefficient $\mathrm{D}_{0}$ and fracture spacing $\underline{2 \mathrm{~B}}$.

Test 1 (Figures 17 20) considers a wide range of free-water diffusion coefficient $D_{0}$, varying from $2.40 \times 10^{-11} \mathrm{~m}^{2} /$ second to $9.31 \times 10^{-9} \mathrm{~m}^{2} /$ second. The fracture spacing $2 B$ varies from $0.1 \mathrm{~m}$ to $10 \mathrm{~m}$. Here the end member $0.1 \mathrm{~m}$ is slightly smaller than the smallest fracture spacing at the RM site.

The following conclusions are drawn from Test 1 :

1) When the fracture spacing is as small as $2 B \leq 0.5 \mathrm{~m}, \mathrm{VfRf}=1.13$. When $2 B>0.5 \mathrm{~m}$, $\mathrm{VfRf}=1.0$. Here VfRf is an effective factor that controls the water volume in fractures in the code RWHet-Pan\&Bodvarsson - scaling the parameter VfRf is equivalent to changing the time step of the mass transfer algorithm. We found that it was necessary to adjust time step of the algorithm to maintain accuracy under the specific condition of small fracture spacing.

2) RWHet-Pan\&Bodvarsson works slightly better than DCPTV2.0 if the free-water diffusion coefficient $D_{0}$ is relatively large. For the small $D_{0}\left(<9.31 \times 10^{-11}\right.$ $\mathrm{m}^{2} /$ second) with a spacing $2 B=0.5 \mathrm{~m}$, RWHet with $\mathrm{VfRf}=1.13$ generates a slightly earlier arrival.

3) RWHet-Pan\&Bodvarsson is valid for a wide range of $D_{0}$. 

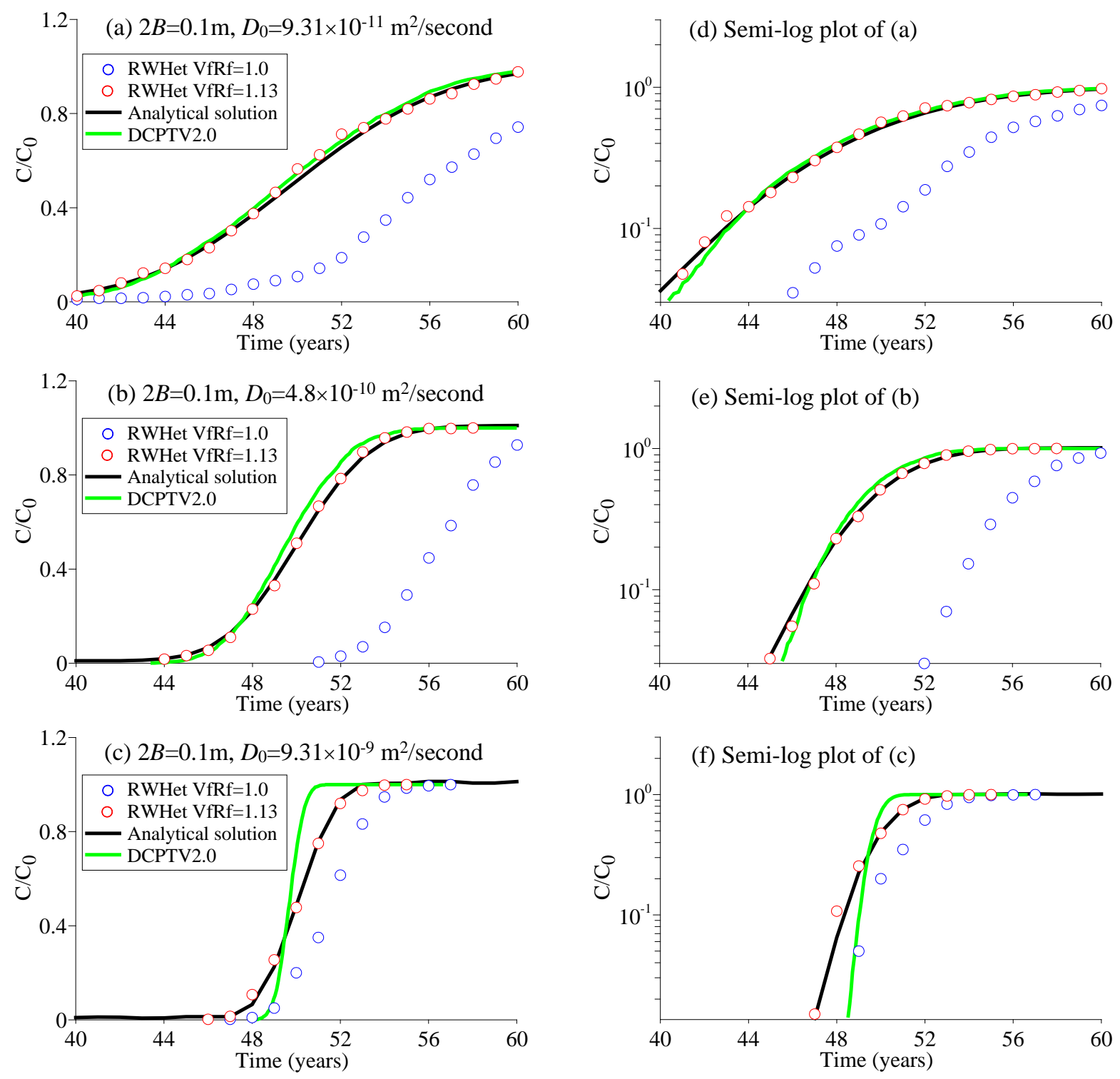

Figure 17. The RWHet-Pan\&Bodvarsson solutions (symbols) versus the analytical solution (black line) and the solution of DCPTV2.0 (green line). This figure shows the influence of the free-water molecular diffusion coefficient $D_{0}$ on BTC. The right plot is the semi-log version of the left plot. $R=1$, aperture $2 b=2 \times 10^{-5} \mathrm{~m}$, and spacing $2 B=0.1 \mathrm{~m}$. 

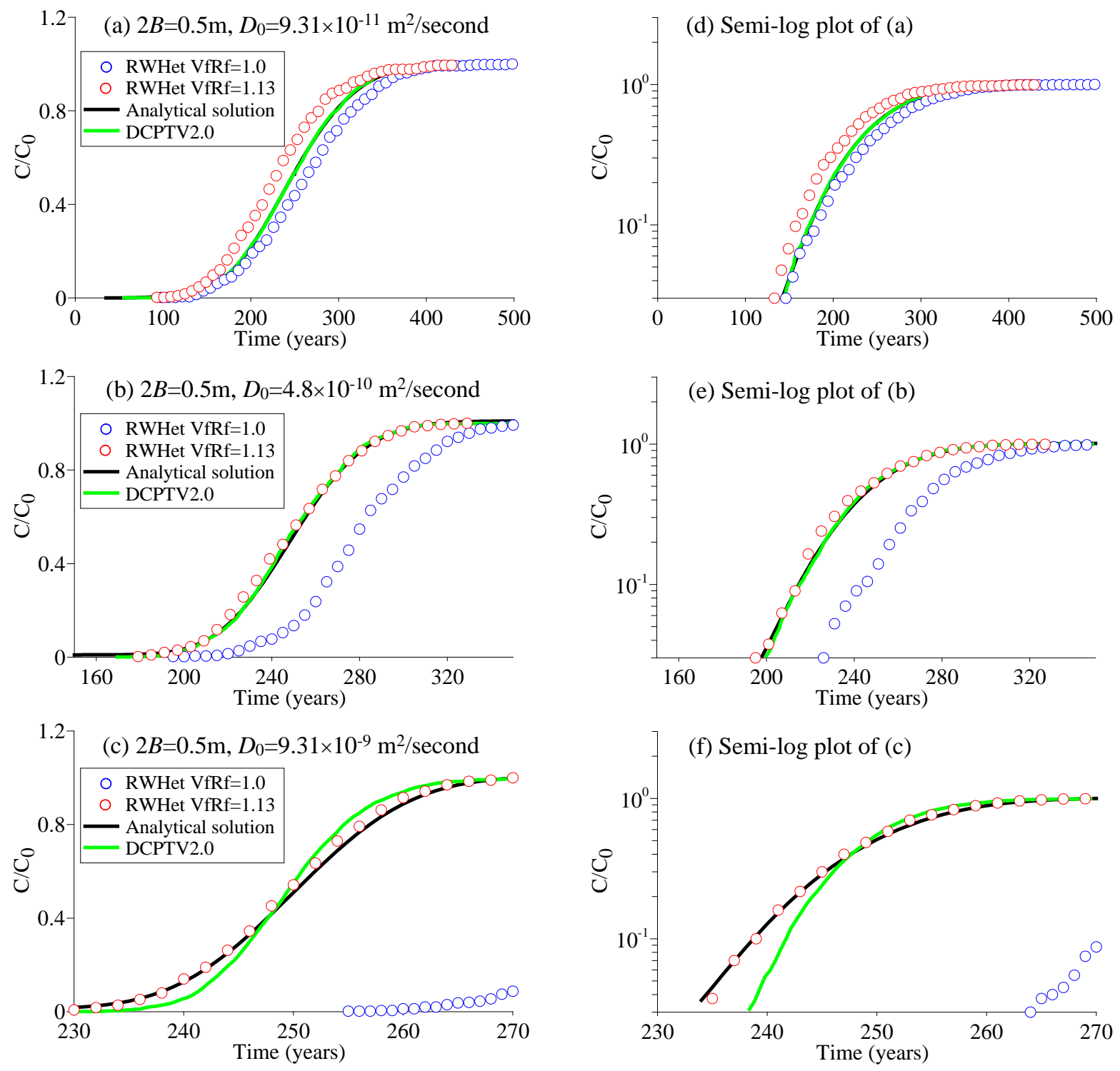

Figure 18. Tests of RWHet-Pan\&Bodvarsson: Influence of the free-water molecular diffusion coefficient $D_{0}$ on BTC. The bottom plot is the semi-log version of the top plot. $R=1$, aperture $2 b=2 \times 10^{-5} \mathrm{~m}$, and spacing $2 B=0.5 \mathrm{~m}$. 

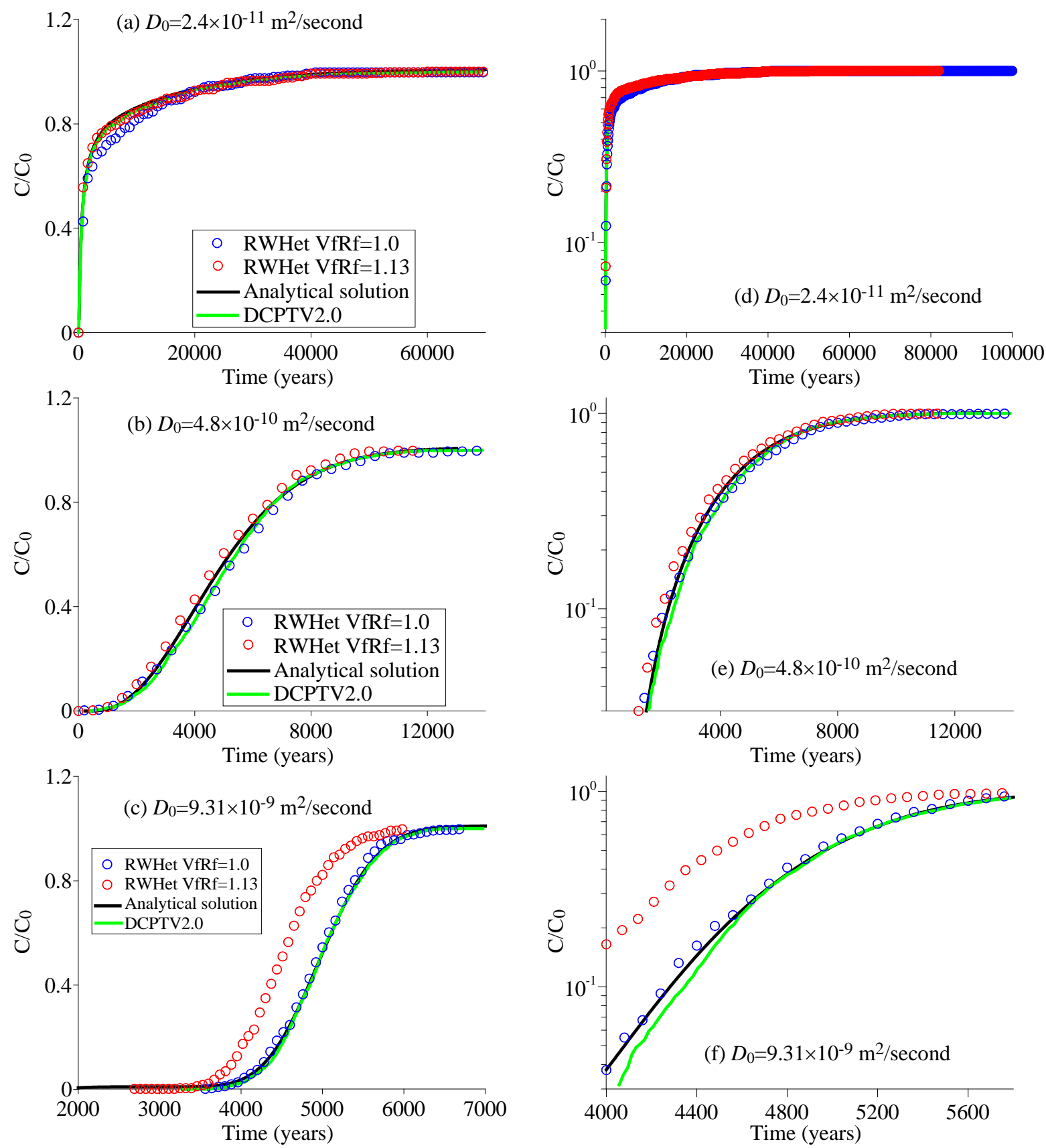

Figure 19. Tests of RWHet-Pan\&Bodvarsson: $\left({ }^{3} \mathrm{H}\right.$ with various $\left.D\right)$ Influence of the free-water molecular diffusion coefficient $D_{0}$ on BTC. The right plot is the semi-log version of the left plot. $R=1$, aperture $2 b=2 \times 10^{-5} \mathrm{~m}$, and spacing $2 B=10 \mathrm{~m}$. 

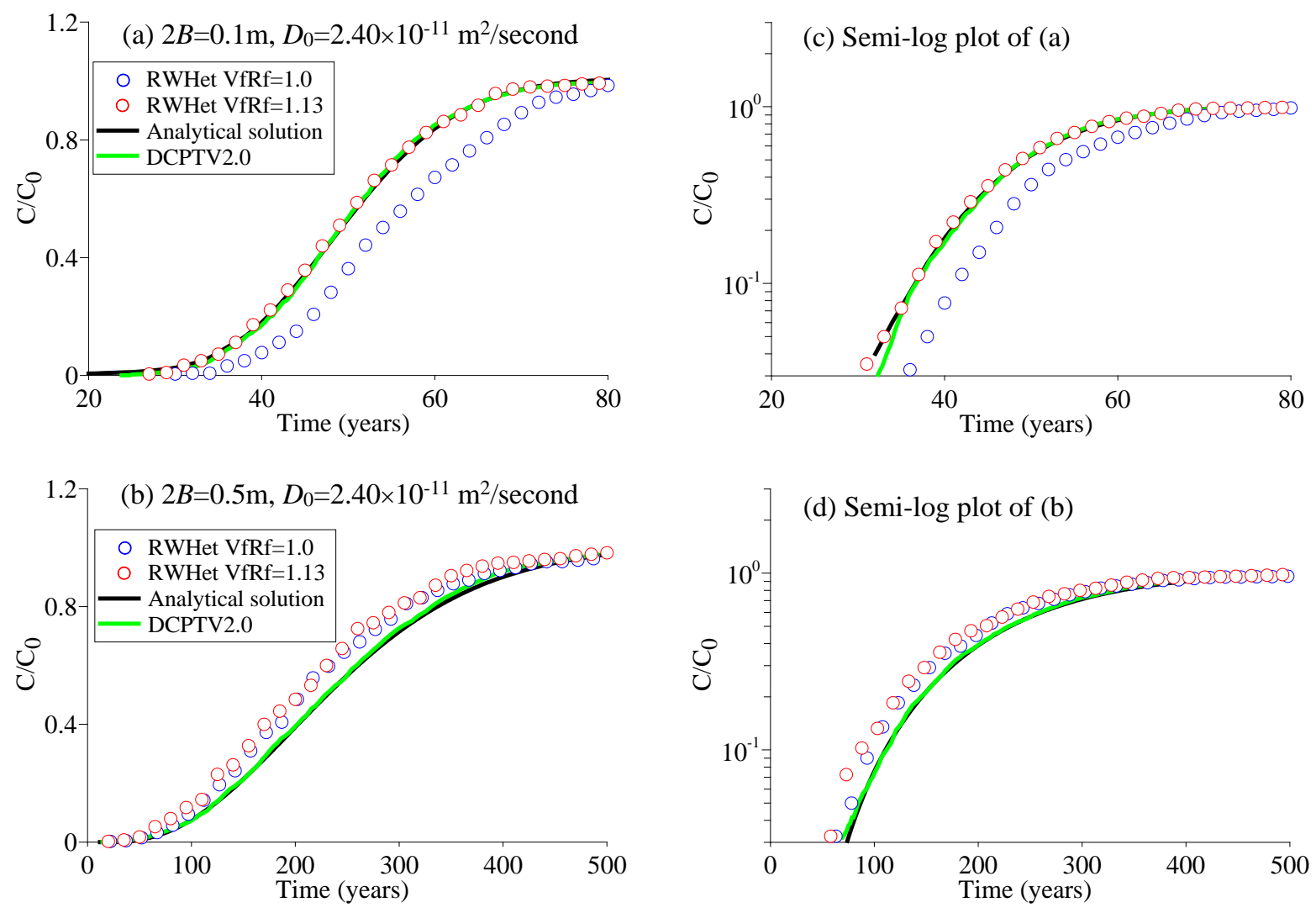

Figure 20. Tests of RWHet-Pan\&Bodvarsson: The smallest $D_{0}\left(=2.40 \times 10^{-11} \mathrm{~m}^{2} / \mathrm{second}\right)$ (freewater diffusion coefficient) for ${ }^{3} \mathrm{H}: R=1$, aperture $2 b=2 \times 10^{-5} \mathrm{~m}$, and spacing $2 B=0.1 \mathrm{~m}$ (top plots) or $0.5 \mathrm{~m}$ (bottom plots). In all plots, the DCPTV2.0 solution is almost identical to the analytical solution, showing that the DCPTV2.0 works well for a small $D_{0}$. The right plot is the semi-log version of the left plot, to show the early time behavior of BTC.

\subsubsection{Test 2: Variable fracture spacing $2 \mathrm{~B}$ (from $0.1 \mathrm{~m}$ to $20 \mathrm{~m}$ )}

In Test 2, we check the influence of fracture spacing (Figures 21 and 22) on BTC. 

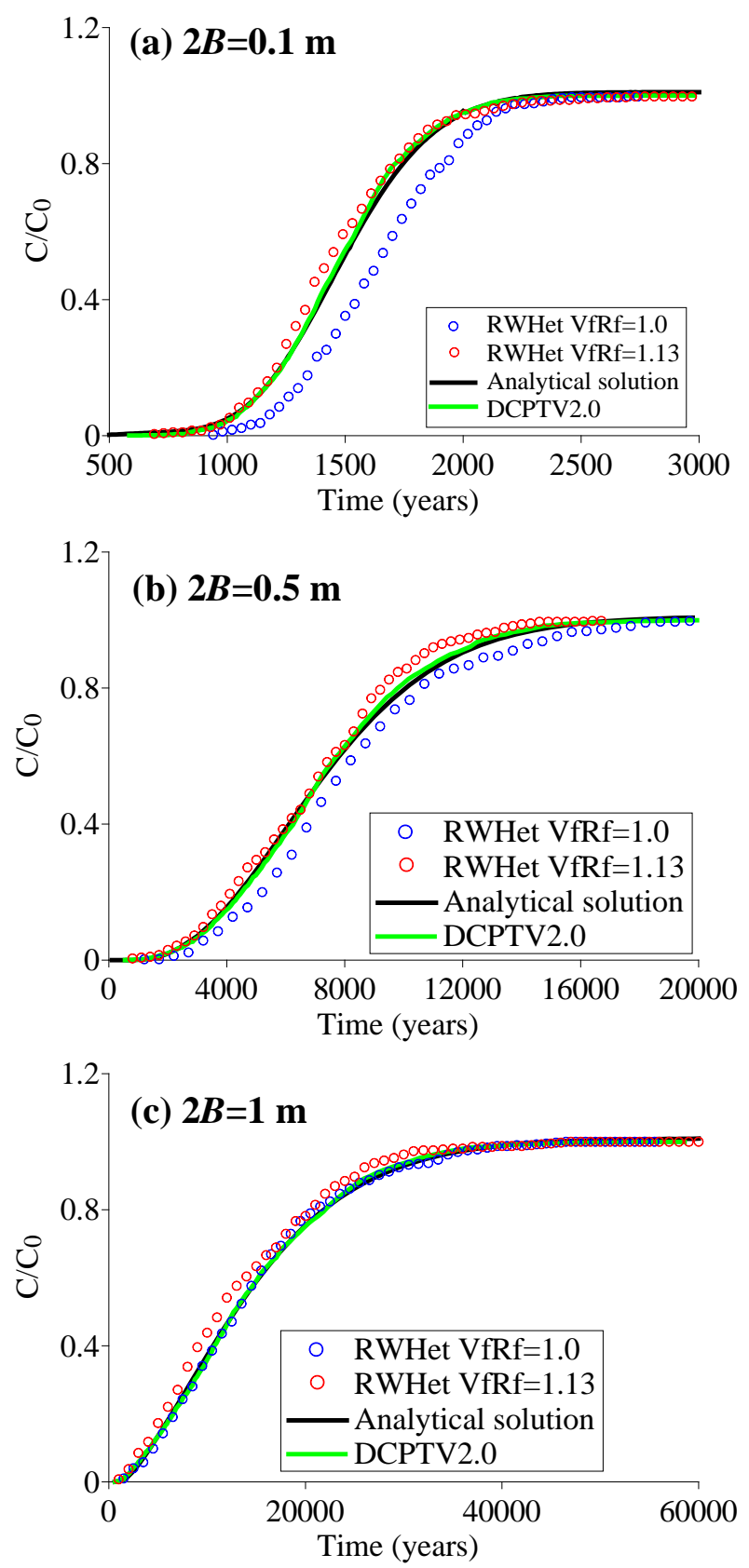
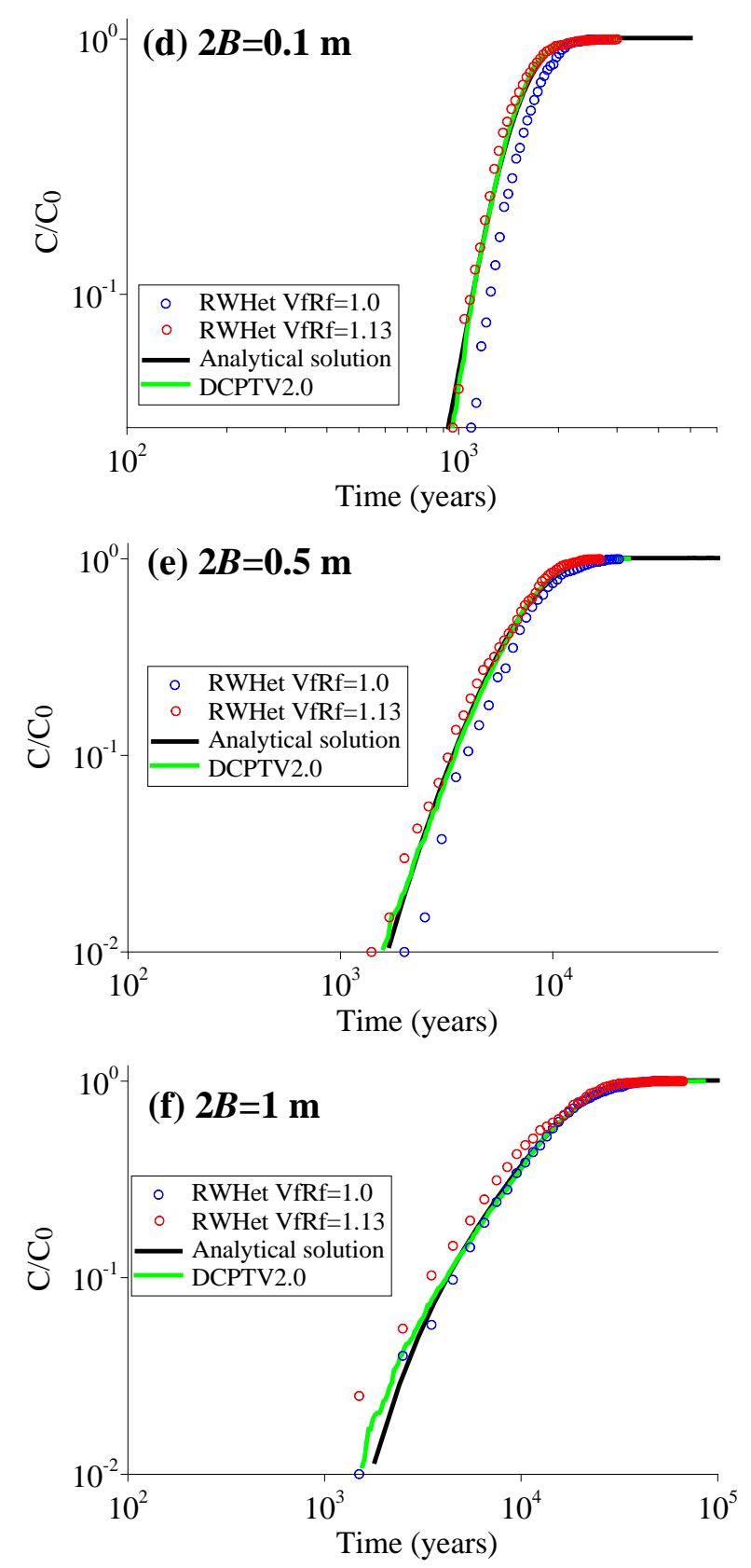

Figure 21. Tests of RWHet-Pan\&Bodvarsson: Influence of the fracture spacing on BTC. The right plot is the log-log version of the left plot. $R_{f}=1, R_{m}=30$, aperture $2 b=2 \times 10^{-5}$ $\mathrm{m}$, and the free-water diffusion coefficient $D_{0}=2.50 \times 10^{-11} \mathrm{~m}^{2} / \mathrm{second}$. 

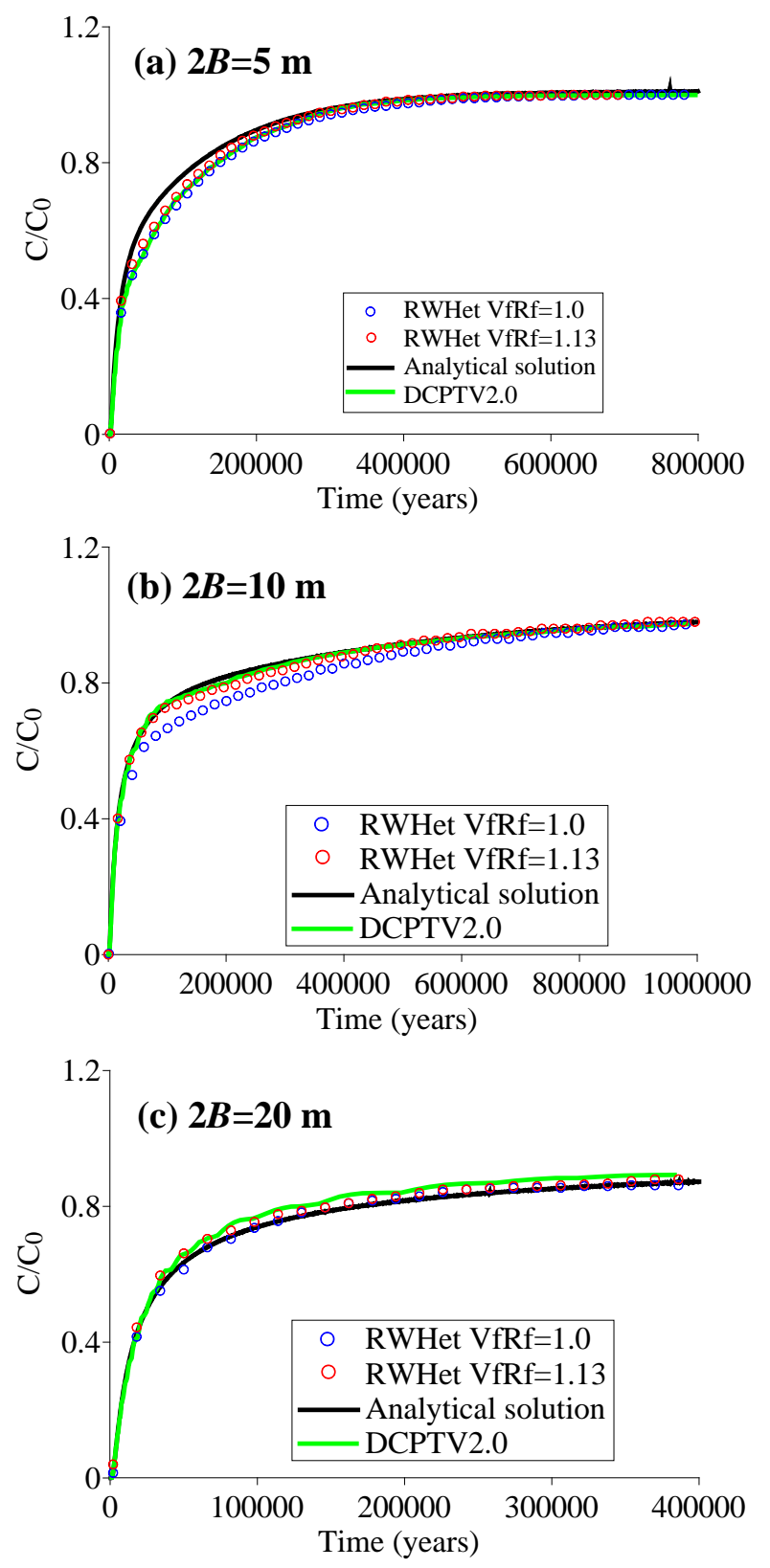
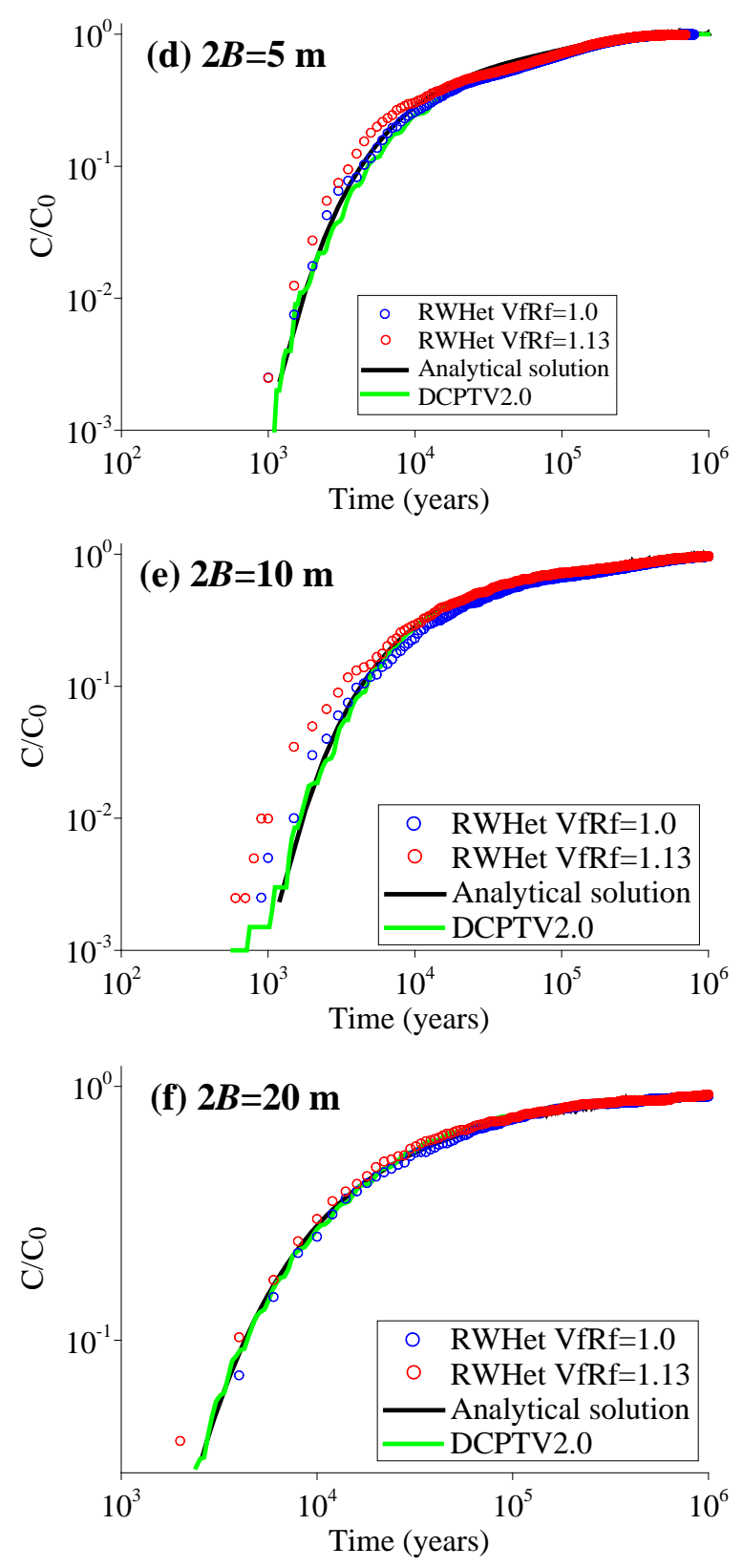

Figure 22. Tests of RWHet-Pan\&Bodvarsson: Influence of the fracture spacing on BTC. The right plot is the log-log version of the left plot. $R_{f}=1, R_{m}=30$, aperture $2 b=2 \times 10^{-5}$ $\mathrm{m}$, and the free-water diffusion coefficient $D_{0}=2.50 \times 10^{-11} \mathrm{~m}^{2} / \mathrm{second}$.

Test-2 (Figure 21 and Figure 22) reveals the following results:

1) When the fracture spacing is as small as $2 B \leq 0.5 \mathrm{~m}, \mathrm{VfRf}=1.13$. When $2 B>0.5 \mathrm{~m}$, $\mathrm{VfRf}=1.0$.

2) RWHet-Pan\&Bodvarsson is valid for a large range of fracture spacing. 


\subsubsection{Test 3: Variable retardation coefficients.}

This test checks the applicability of RWHet-Pan\&Bodvarsson for various retardation coefficients (Figure 23).
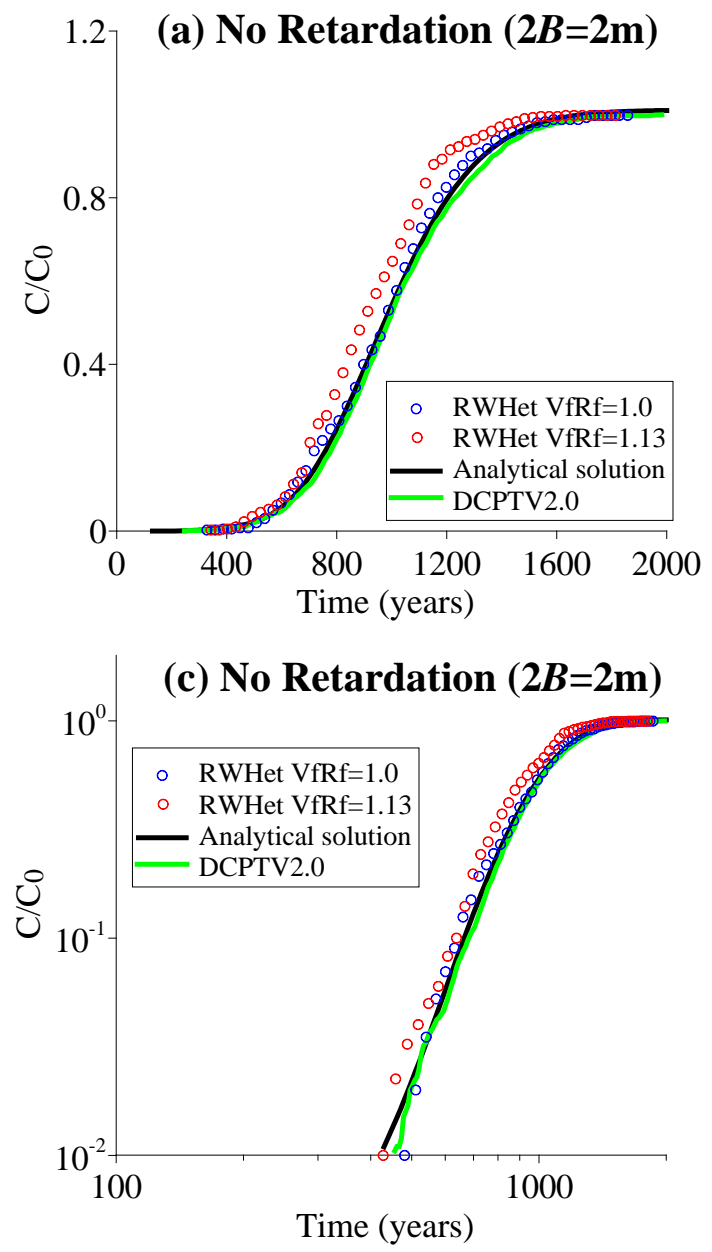
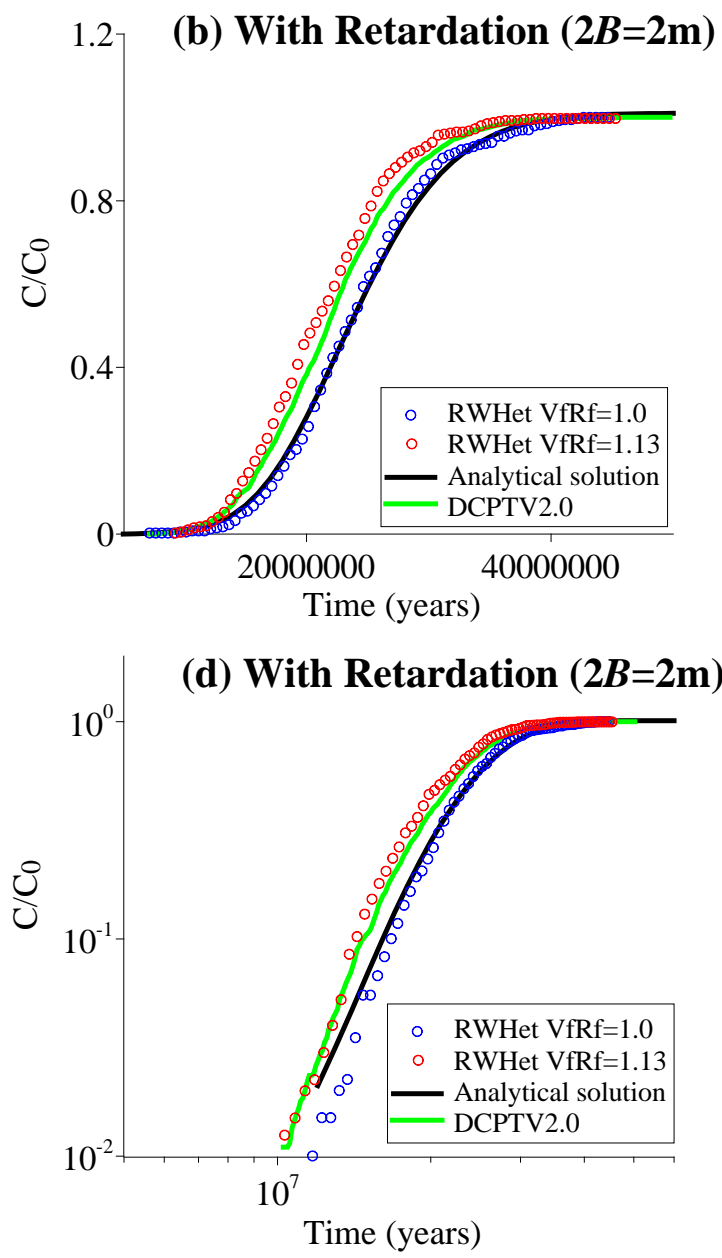

Figure 23. Tests of RWHet-Pan\&Bodvarsson: Influence of the retardation coefficient on BTC. The bottom plot is the log-log version of the top plot. In (a) and (c), $R_{f}=R_{m}=1$. In (b) and (d), $R_{f}=11.59$, and $R_{m}=24193.0$ (retardation based on $k_{d}$ values for ${ }^{241} \mathrm{Am}$ from volcanic tuff units). The other parameters are: fracture spacing $2 B=2 \mathrm{~m}$, aperture $2 b=2.0 \times 10^{-5} \mathrm{~m}$, and the free-water diffusion coefficient $D_{0}=3.06 \times 10^{-10}$ $\mathrm{m}^{2} /$ second (for ${ }^{241} \mathrm{Am}$ ).

Test-3 (Figure 23) has the following conclusions:

1) RWHet-Pan\&Bodvarsson is valid for a very wide range of retardation coefficients.

2) Here the fracture spacing $2 B>0.5 \mathrm{~m}$, so that $\mathrm{VfRf}=1.0$ is selected.

\subsubsection{Test 4: Variable fracture aperture $2 \mathrm{~b}$ (from $2 \mathrm{~b}=2 \times 10^{-5} \mathrm{~m}$ to $2 \mathrm{~b}=1 \times 10^{-3} \mathrm{~m}$ )}

Here we test the variation of fracture aperture. The solution of DCPTV2.0 is not listed for this case, due to inadequate documentation on how to adjust the fracture aperture in the User's Manual of DCPTV2.0. 

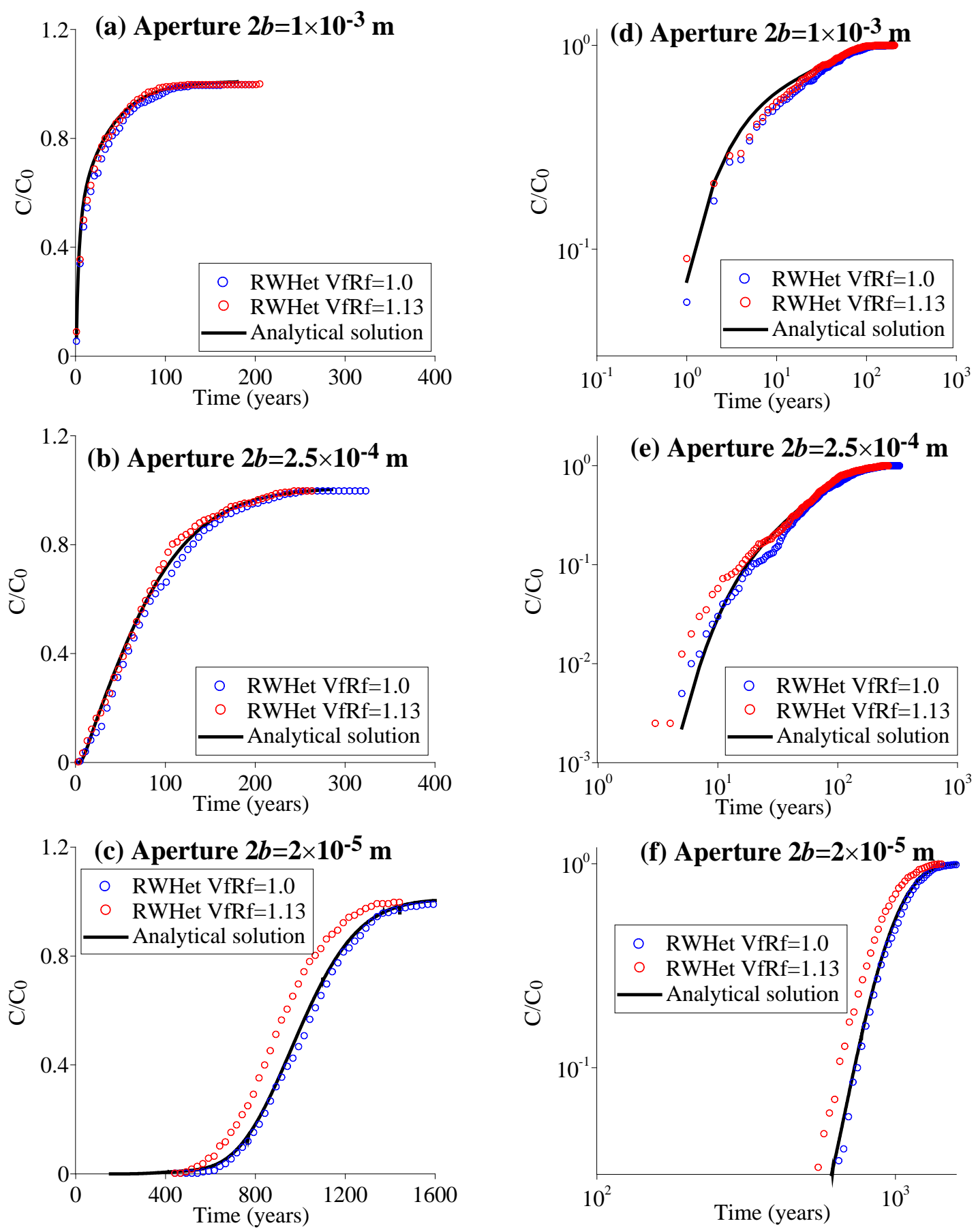

Figure 24. Tests of RWHet-Pan\&Bodvarsson: Influence of the fracture aperture on BTC. The right plot is the log-log version of the left plot. $R_{f}=1, R_{m}=1$, fracture spacing $2 B=2$ $\mathrm{m}$, and the free-water diffusion coefficient $D_{0}=4.80 \times 10^{-10} \mathrm{~m}^{2} /$ second.

Test-4 (Figure 24) shows:

1) RWHet-Pan\&Bodvarsson is valid for a large range of fracture aperture.

2) In the experiments, the fracture spacing is $2 B=2 \mathrm{~m}>0.5 \mathrm{~m}$, and therefore VfRf $=1.0$. 


\subsubsection{Test 5}

In this test, we apply the updated RWHet-Pan\&Bodvarsson to simulate the previous numerical experiments that cannot be captured by the earlier version RWHet3.25_NUFT.f90 (see Figure 7 and Figure 9). Results (Figure 25 and Figure 26) show that RWHet-Pan\&\&Bodvarsson improves RWHet3.25_NUFT.f90 and matches the analytical solution.
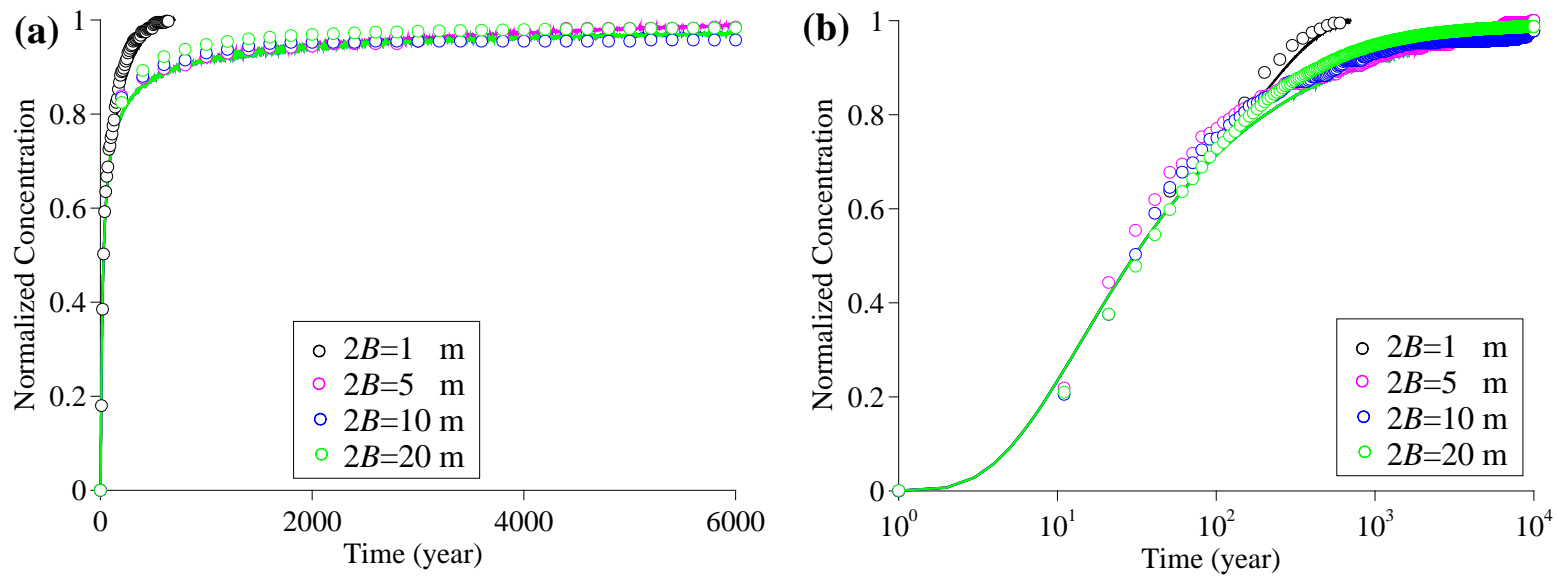

Figure 25. Tests of RWHet-Pan\&Bodvarsson: Updated version of Figure 7: The simulated solution (blue symbols) versus the analytical solution (the red line) of tracer breakthrough curves, where the model parameters are the same as Figure 7. The right column is the semi-log plot of the left column, to show the tail at early times. 

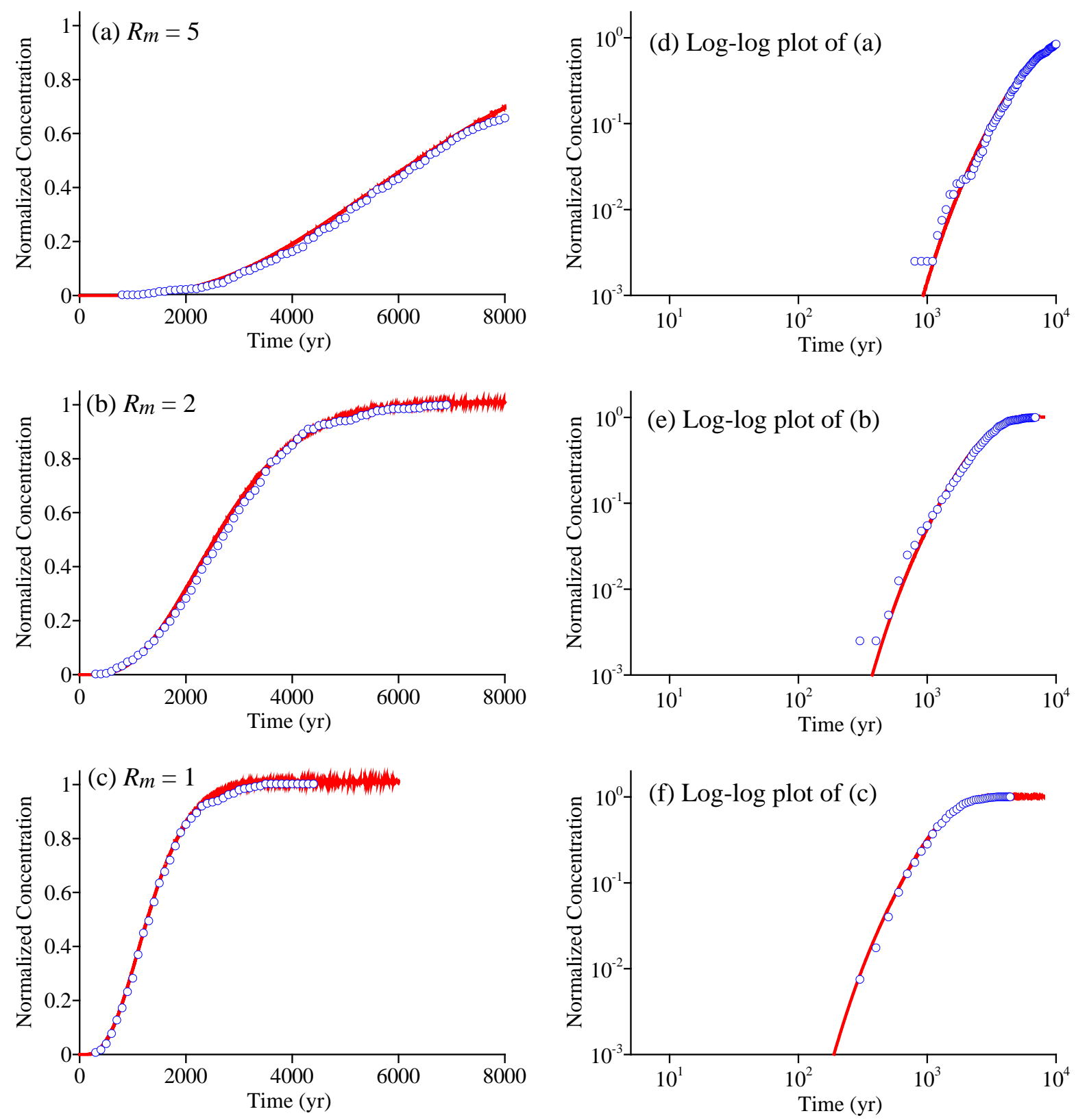

Figure 26. Tests of RWHet-Pan\&Bodvarsson: Updated version of Figure 9: The numerical solution (blue symbols) versus the analytical solution (the red line) of tracer breakthrough curves, where the model parameters are the same as Figure 9, except for the matrix retardation coefficient $R_{m}$. The right column is the log-log plot of the left column, to show the tail at early times.

In summary, Pan and Bodvarsson's [2002] method was tested for single fracture and parallel fracture systems and shown to be both computationally efficient and perform well over a wide range of diffusion coefficients (3 orders of magnitude), fracture spacings ( 0.1 to 20 meters), fracture aperture (3 orders of magnitude), retardation coefficients (4 orders of magnitude in $R_{m}$ ), and velocities (4 orders of magnitude, see Figure 27). Thus, it 
is our conclusion that the Pan and Bodvarsson [2002] approach is suitable for UGTA applications as it has been tested over the full parameter range considered likely for the Nevada National Security Site.
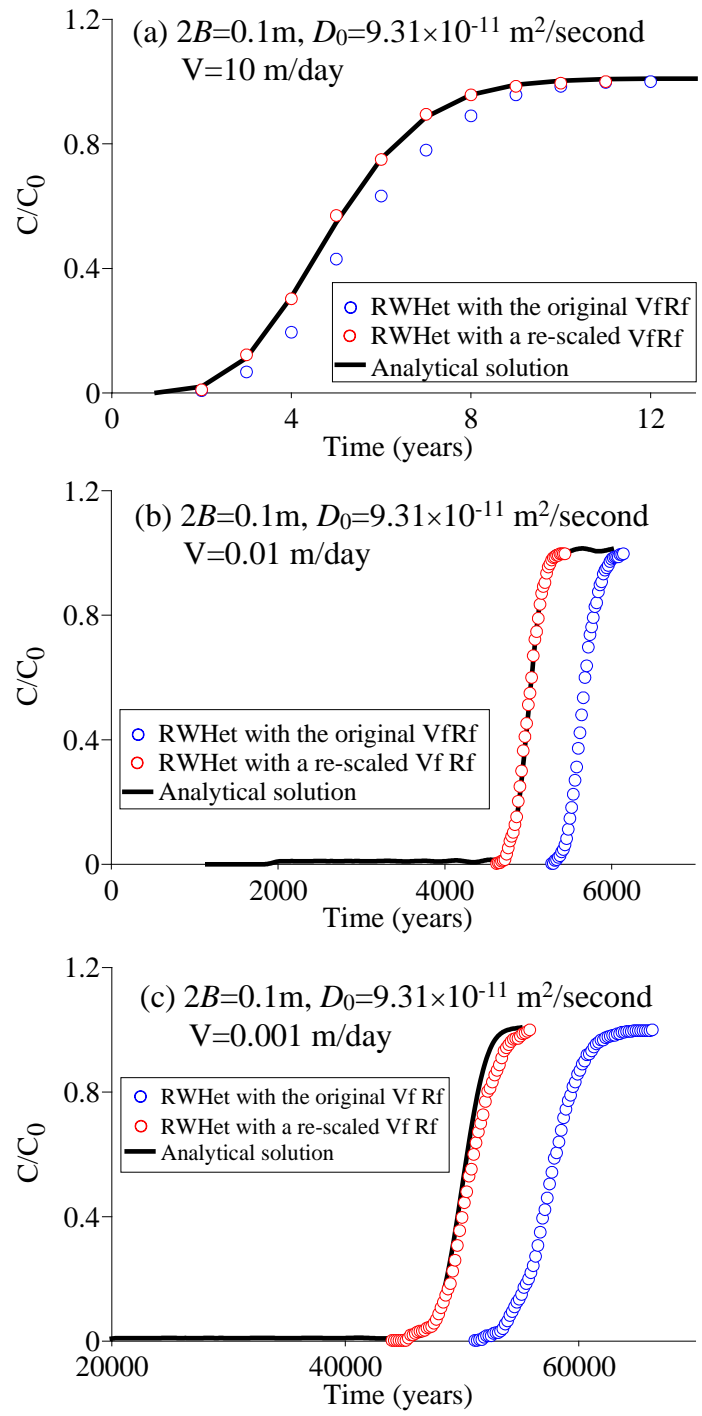

(d) Semi-log plot of (a)

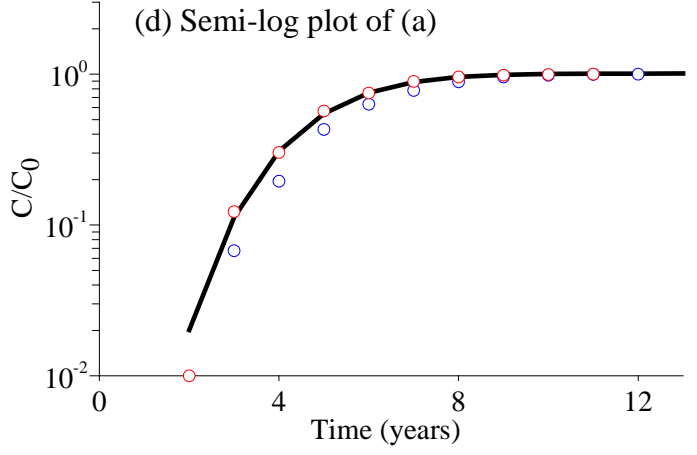

(e) Semi-log plot of (b)
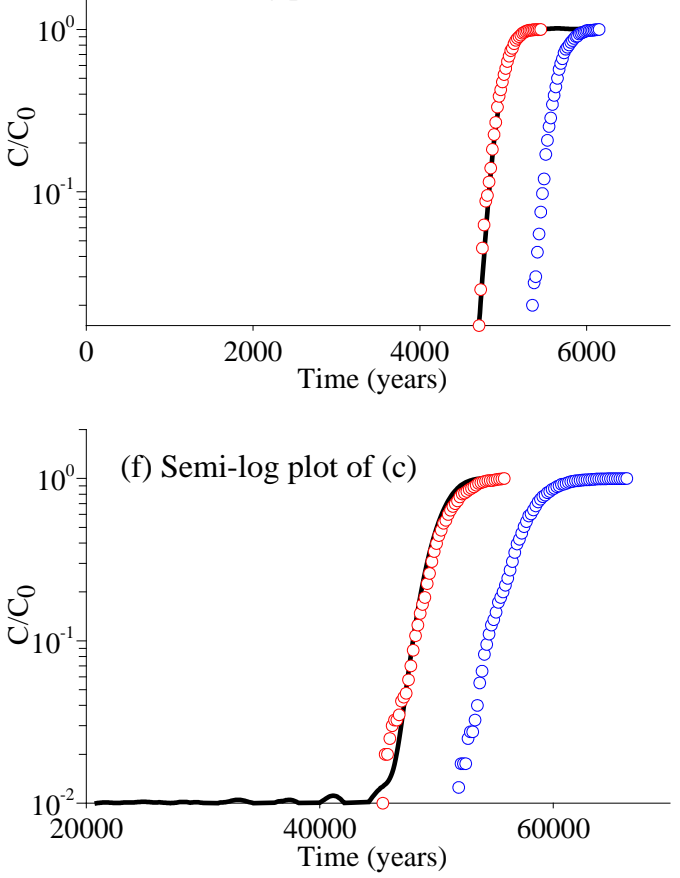

Figure 27. Tests of RWHet-Pan\&Bodvarsson: (non-sorbing radionuclide subject with a 4 order magnitude in velocity $V$ ) show that the VfRf correction is insensitive to velocity. The right plot is the semi-log version of the left plot. Retardation coefficient $R=1$, aperture $2 b=2 \times 10^{-5} \mathrm{~m}$, the free-water molecular diffusion coefficient $D_{0}=9.31 \times 10^{-11}$ meter $^{2} /$ second, and fracture spacing $2 B=0.1 \mathrm{~m}$. 


\section{CONCLUSIONS}

We applied and evaluated four different numerical methods to simulate contaminant transport through fractured porous media using a fully Lagrangian solver. The code RWHet was updated by adding additional components to account for the particle tracking though fracture and matrix continua with mass transfer.

We first developed the Lagrangian methods based on the transfer probability approach proposed by Liu et al. [2000], and coded it into RWHet. Numerical examples show that this method is limited to certain ranges of parameters (for example, the freewater molecular diffusion coefficient $D_{0}$ must be relatively large), due to the intrinsic assumption in building the transfer probability.

To address the above limitations, we then developed a direct numerical simulation reflective (DNS-Reflective) method. The novel DNS-Reflective method can track directly the particle dynamics across the fracture/matrix interface, without any empirical assumptions. This advantage should make the DNS-Reflective method feasible for a wide range of parameters. Numerical tests of the DNS-Reflective, however, show that the method is computationally very demanding, since the time step must be very small for particles to experience both the fracture and matrix.

To improve the computational efficiency, we then adopted Roubinet et al.'s method [2009], which uses the first passage time distributions to simulate dual-domain mass transfer. The DNS-Roubinet method was found to be computationally efficient, but it fails to capture solute transport through a parallel fracture system with small fracture spacing.

Finally, we adopted the transient range approach proposed by Pan and Bodvarsson [2002] in RWHet. Numerical results were checked against analytical solutions, and also compared to DCPTV2.0 [Pan, 2002]. This version of RWHet (called RWHet-Pan\&Bodvarsson) can capture contaminant transport in fractured porous media for a full range of transport parameters. This is the most effective and efficient solution since 1) the time step needs not to be very small to resolve particle transfer between fractures and matrix blocks, and 2) it does not truncate the first passage time distribution (note the truncation can create apparent errors when the fracture spacing is small).

\section{REFERENCES}

Ahlstrom, S. W., H. P. Foote, R. C. Arnett, C. R. Cole, and R. J. Serne, Multi-component mass transport model: Theory and numerical implementation. Report BNWL-2127. Battelle Pacific Northwest Laboratory, Richlnd, WA, 1977.

Bechtold, M., J. Vanderborght, O. Ippisch, and H. Vereecken, Efficient random walk particle tracking algorithm for advective-dispersive transport in media with discontinuous dispersion coefficients and water contents, Water Resources Research, 47, W10526, doi:10.1029/2010WR010267, 2011.

Carle, S. F., A transition probability-based approach to geostatistical characterization of hydrostratigraphic architecture, Ph.D. Dissertation, University of California, Davis, 248 pp., 1996. 
Carle, S. F., T-PROGS: Transition Probability Geostatistical Software, University of California, Davis, 84 pp., 1999.

Carslaw, H. S., and J. C. Jaeger, Conduction of Heat in Solids, Clarendon, Oxford, U. K., 363-365 pp., 1959.

Cordes, C., H. Daniels, and G. Rouvé, A new very efficient algorithm for particle tracking in layered aquifers, In: Computer Methods in Water Resources II, Vol. 1, Groundwater Modeling and Pressure Flow, edited by D. B. Sari, C. A. Brebbia, and D. Ouazar, 41-55, Springer-Verlag, New York, 1991.

Crank, J., The Mathematics of Diffusion, $2^{\text {nd }}$ ed., Oxford University Press, New York, 414 pp., 1975.

Gelhar, L. W., and M. A. Collins, General analysis of longitudinal dispersion in nonuniform flow, Water Resources Research, 7(6), 1511-1521, 1971.

Green, C. T., J. K. BÄohlke, B. A. Bekins, and S. P. Phillips, Mixing effects on apparent reaction rates and isotope fractionation during denitrification in a heterogeneous aquifer, Water Resources Research, 46, W08525, doi:10.1029/2009WR008903, 2010.

Haggerty, R., S. McKenna, and L. Meigs, On the late-time behavior of tracer test breakthrough curves, Water Resources Research, 36(12), doi:10.1029/2000WR9000214, 2000.

Harbaugh, A. W., and M. G. McDonald, User's documentation for MODFLOW-96, an update to the U.S. Geological Survey modular finite difference ground-water flow model, USGS Open File Report, Reston, Virginia: USGS, 1996.

Hassan, A. E., Comment on "Determination of particle transfer in random walk particle methods for fractured porous media" by H. H. Liu et al., Water Resources Research, 38(11), W1221, doi:10.1029/2000WR000132, 2002.

Javandel, I., C. Doughty, and C. F. Tsang, Groundwater Transport: Handbook of Mathematical Models, American Geophysical Union Water Resources Monogram, 10, 228 pp., 1984.

Kinzelbach, W., The random walk method in pollutant transport simulation, In: Groundwater Flow and Quality Modeling, E. Custodio et al. (eds.), Reidel Publishing Company, 227-245, 1988.

LaBolle, E. M., G. E. Fogg, and A. F. B. Tompson, Random-walk simulation of transport in heterogeneous porous media: Local mass conservation problem and implementation methods, Water Resources Research, 32(3), 583-593, 1996.

LaBolle, E. M., J. Quastel, G. E. Fogg, and J. Gravner, Diffusion processes in composite porous media and their numerical integration by random walks: Generalized stochastic differential equations with discontinuous coefficients, Water Resources Research, 36(3), 651-662, 2000.

LaBolle, E. M., and G. E. Fogg, Role of molecular diffusion in contaminant migration and recovery in an alluvial aquifer system, Transport in Porous Media, 42, 155-179, 2001. 
LaBolle, E. M., and Y. Zhang, Reply to Comment by Doo-Hyun Lim on "Diffusion processes in composite porous media and their numerical integration by random walks: Generalized stochastic differential equations with discontinuous coefficients." Water Resources Research, 42, W02602, doi:10.1029/2005WR004403, 2006.

LaBolle, E. M., RWHet: Random Walk Particle Model for Simulating Transport in Heterogeneous Permeable Media, Version 3.2, User's Manual and Program Documentation, 2006.

Liu, H., H., G. S. Bodvarsson, and L. Pan, Determination of particle transfer in random walk particle methods for fractured porous media, Water Resources Research, 36(3), 707-713, 2000.

Mahinthakumar, G., and A. J. Valocchi, Application of the connection machine to flow and transport problems in 3-dimensional heterogeneous aquifers, Advances in Water Resources, 15(5), 289-302, 1992.

Maxwell, R. M., and A. F. B. Tompson, SLIM-FAST: A User's Manual, Lawrence Livermore National Laboratory, UCRL-SM-225092, 49 pp., 2006.

Maxwell, R. M., C. Welty, and R. W. Harvey, Revisiting the Cape Cod bacteria injection experiment using a stochastic modeling approach, Environmental Science and Technology, 41(15), 5548-5558, 2007.

Moench, A. F., and A. Ogata, A numerical inversion of the Laplace transform solution to radial dispersion in a porous medium, Water Resources Research, 17(1), 250-253, 1981.

Nitao, J. J., Some examples of the application and validation of the NUFT subsurface flow and transport code, Lawrence Livermore National Laboratory, UCRL-ID145163, August, 2001.

Painter, S. L., User's Manual for Walkabout Version 1.0, LA-UR-11-01952, 29 pp, 2011.

Pan, L. H., H. H. Liu, M. Cushey, and G. Bodvarsson, DCPT v1.0 - New Particle Tracker for Modeling Transport in Dual-Continuum Media, User's Manual, LBNL, 47 pp., April, 2001.

Pan, L. H., User Manual (UM) for DCPT v2.0, SAN: LBNL-2001-230, 15 pp., 2002.

Pan, L. H., and G. Bodvarsson, Modeling transport in fractured porous media with the random-walk particle method: The transient activity range and the particle transfer probability, Water Resources Research, 38(6), 10.1029/2001WR000901, 2002.

Prickett, T. A., T. G. Naymik, and C. G. Longquist, A random walk solute transport model for selected groundwater quality evaluations, Report I-11. Illinois State Water Survey, Urbana, 1981.

Pohlmann, K., G. Pohll, J. Chapman, A. E. Hassan, R. Carroll, and C. Shirley, Modeling to Support Groundwater Contaminant Boundaries for the Shoal Underground Nuclear Test, Report DOE/NV/13609-13, 197 pp., 2004. 
Roubinet, D., H. Liu, Q. Zhou, and J. de Dreuzy, Influence of the fracture-network structure on contaminant transport in fractured porous media, American Geophysical Union, Fall Meeting 2009, Abstract \#H23D-0985, 2009.

Roubinet, D., J.-R. de Dreuzy, and D. M. Tartakovsky, Semi-analytical solutions for solute transport and exchange in fractured porous media, Water Resources Research, in press, 2012.

Semra, K., P. Ackerer, and R. Mose, Three dimensional groundwater quality modeling in heterogeneous media. In: Water Pollution II, Modeling, Measuring and Prediction, Mass Comput. Mech., Billerica, 3-11, 1993.

Sudicky, E. A., and E. O. Frind, Contaminant transport in fractured porous media: Analytical solutions for a system of parallel fractures, Water Resources Research, 18(6), 1634-1642, 1982.

Tang, D. H., E. O. Frind, and E. A. Sudicky, Contaminant transport in fractured porous media: Analytical solution for a single fracture, Water Resources Research, 17(3), 555-564, 1981.

Tompson, A. F. B., Numerical simulation of solute transport in three-dimensional randomly heterogeneous porous media, Water Resources Research, 29(11), 37093726, 1993.

Tsang, Y. W., and C. F. Tsang, A particle-tracking method for advective transport in fractures with diffusion into matrix blocks, Water Resources Research, 37(3), 831835, 2001.

Uffink, G. J. M., A random walk method for the simulation of macrodispersion in a stratified aquifer, In: Relation of Groundwater Quality and Quantity, IAHS Publ., 146, 103-114, 1985.

Van Genuchten, M. Th., and W. J. Alves, Analytical solutions of the one-dimensional convective-dispersion solute transport equation, U. S. Department of Agriculture Technical Bulletin, No. 1661, 151 pp., 1982.

Weissmann, G. S., Y. Zhang, E. M. LaBolle, and G. E. Fogg, Dispersion of groundwater age in an alluvial aquifer system, Water Resources Research, 38(10), doi: 10.1029/2001WR000907, 2002.

Wels, C., L. Smith, and R. Beckie, The influence of surface sorption on dispersion in parallel plate fractures, Journal of Contaminant Hydrology, 28, 95-114, 1997.

Yamashita, R., and H. Kimura, Particle-tracking technique for nuclide decay chain transport in fractured porous media, Journal of Nuclear Science and Technology, 27, 1042-1049, 1990.

Zheng, C. M., MT3D: A Modular Three-Dimensional Transport Model, Documentation and User's Guide, 1992.

Zheng, C. M., and P. P. Wang, MT3DMS: A Modular Three-Dimensional Multispecies Transport Model, Documentation and User’s Guide, 1999. 
Zyvoloski G., FEHM: A control volume finite element code for simulating subsurface multi-phase multi-fluid head and mass transfer, Los Alamos National Laboratory Report LA-UR-07-3359, Los Alamos, New Mexico, USA, 2007. 


\section{APPENDIX A: VALIDATION OF RWHet}

The goal of this appendix is to test RWHet [LaBolle, 2006]. Six examples are selected carefully and described in detail in the following sections.

We first check RWHet solutions against simple cases where the analytical solutions are available. This leads to Example 1, which is also the commonly used example for checking the applicability of a Lagrangian solver [Zheng and Wang, 1999; LaBolle, 2006].

Example 2 and Example 3 calculate particle motions in two-dimensional (2-d) homogenous boxes, where the flow field may be divergent and/or convergent. The two examples were also used by Zheng [1992] and Zheng and Wang [1999] to test the applicability of the particle tracking component in the code MT3D. LaBolle [2006] also compared the analytical solutions to RWHet solutions. Here we extend the RWHet simulation in LaBolle [2006] by evaluating the evolution of particle trajectories in the divergent/convergent flow fields, which can further check the capability of RWHet in capturing the flow path in heterogeneous media.

Example 4 and Example 5 calculate particle motions in two-dimensional heterogeneous boxes with increasing complexity of medium heterogeneity. The correct interpolation of particle velocities in a composite medium is the core of a typical Lagrangian solver. Example 4 was first built by Zheng [1992] and used also by Zheng and Wang [1999] to check the capability of MT3D in simulating contaminant transport through a composite heterogeneous medium. Here we extend their work by evaluating the response of streamlines to the convergent/divergent flow fields with various injecting/pumping rates. Example 5 contains a high-resolution hydrofacies model. RWHet is used to simulate particle dynamics across discrete interfaces of both velocity and dispersion coefficient. The similar modeling approach as Example 5, including the modeling of subsurface heterogeneity and solute transport, had also been applied and validated by LaBolle and Fogg [2001] and Green et al. [2010].

Example 6 is the Verification Test 3 used by Painter [2011] for the new code Walkabout. This example provides the direct comparison between Walkabout and RWHet.

\section{A.1. Validation of RWHet using one-dimensional transport tests: Advection, dispersion, sorption and decay (Example 1)}

Example 1 - Similar to Zheng and Wang [1999], we first test the capability of RWHet in capturing tracer transport in a one-dimensional (1-d) homogeneous medium, where the analytical solution is available. Figure 28 shows four cases, involving advection (Case 1), dispersion (Case 2), sorption (Case 3) and decay (Case 4). In all cases, the numerical model consists of 101 columns, 1 row and 1 layer of uniform cells. The cell width is $10 \times 10$ feet along the row and column directions, and the layer thickness is 1 foot. The effective porosity is 0.25 . In the legend, $V$ is the groundwater seepage velocity, $D$ is the dispersion coefficient (i.e., the combined effect of molecular diffusion and mechanical dispersion), $R$ denotes the retardation coefficient, and $\gamma$ is the decay rate constant. In all cases, a constant concentration $(C=1)$ is defined at the first upstream cell, 
representing a continuous source at the inlet. The resultant RWHet solution is the concentration at each cell, using the same spatial discretization. The analytical solution was provided by Van Genuchten and Alves [1982, with the code “ODAST”].
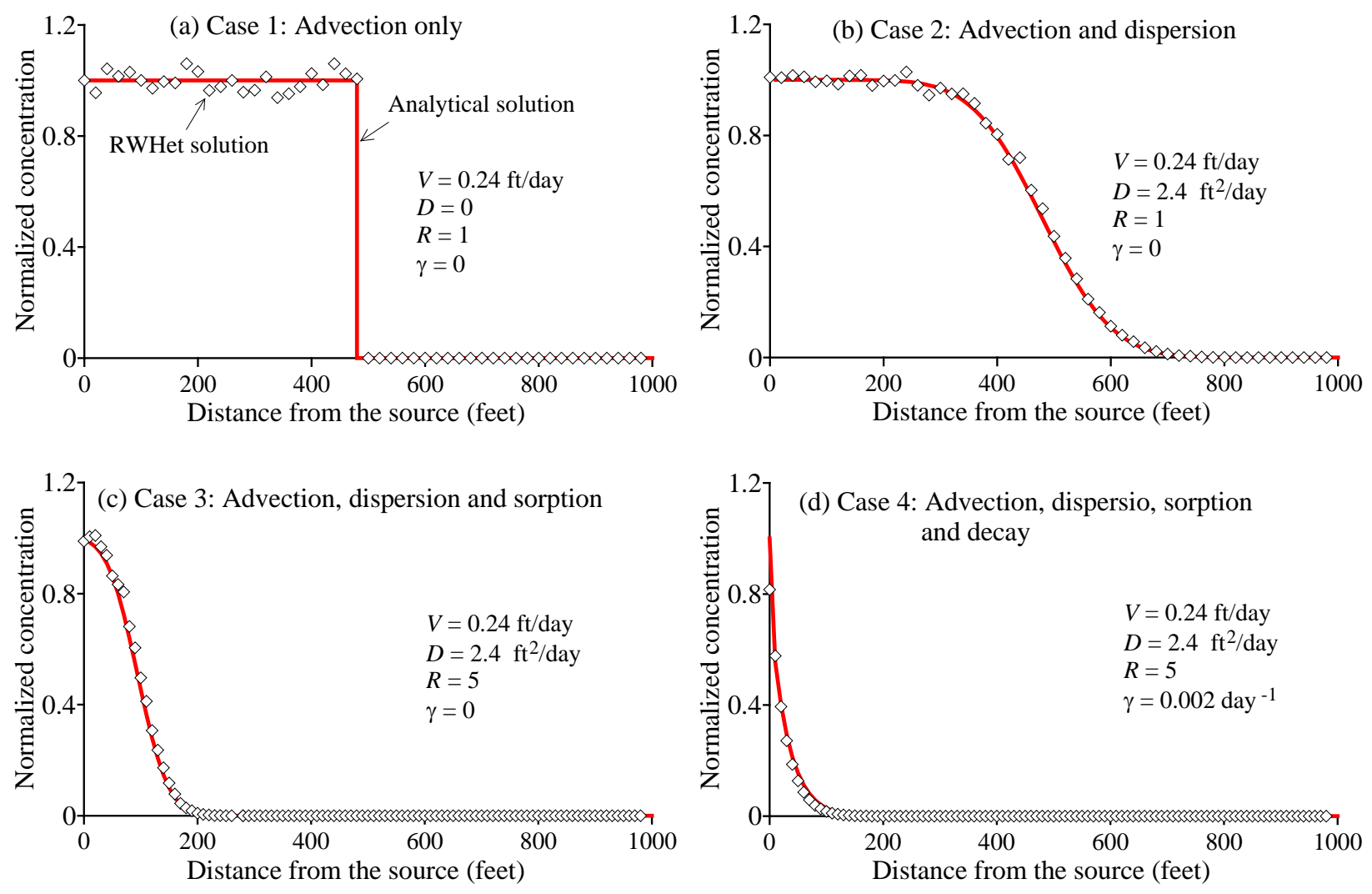

Figure 28. Example 1 - Comparison of the tracer snapshot at time $t=2000$ days using RWHet (symbols) and the analytical solutions (red lines). Adopted from Zheng and Wang [1999, page 7-3]. The average difference between the numerical solution and the analytical solution is $2.02 \times 10^{-3}(\mathrm{a}), 3.17 \times 10^{-3}(\mathrm{~b}), 5.47 \times 10^{-3}(\mathrm{c})$, and $-3.72 \times 10^{-3}(\mathrm{~d})$, respectively.

We first used the USGS code MODFLOW [Harbaugh and McDonald, 1996] to calculate the steady-state flow, and then used the output flux data as the input file to run RWHet. The particle tracking result from RWHet generally matches the analytical solution for all the cases shown in Figure 28. In Case 1, RWHet solutions contain apparent noises (Figure 28(a)). This is the well-known numerical oscillation behavior in the Lagrangian solver, which can be alleviated by increasing the number of released particles (note that the number of particles used in this simulation is 1000). The normalized concentration exceeds one at some cells. Particles are merged at these positions, resulting in a particle number density larger than the average (which is one). For the other three cases, RWHet solutions generally catch the plume snapshots (Figure 28(b), (c), (d)). 


\section{A.2. Two-dimensional homogeneous box: Transport in a radial flow field (Examples 2 and 3)}

Example 2 - This example considers the two-dimensional transport of solute injected from a fully penetrating well [Zheng, 1992, Chapter 7.3]. The injection rate at the well is $100 \mathrm{ft}^{3} /$ day, resulting in a divergent flow field. The initial and boundary conditions for the transport model are:

$$
\begin{array}{rlrl}
C(r, 0) & =0, & \\
C\left(r_{w}, t\right) & =1, & & t>0 \\
\left.\frac{\partial C}{\partial r}\right|_{r \rightarrow \infty} & =0, & t>0
\end{array}
$$

where $r_{w}$ is the well radius. This transport model has an approximate analytical solution [Moench and Ogata, 1981; Javandel et. al., 1984].

Zheng [1992] selected a finite model domain discretized as 31 columns, 31 rows, and 1 layer, where the grid size is $10 \times 10 \times 1 \mathrm{ft}$ along $\mathrm{x} / \mathrm{y} / \mathrm{z}$ directions, respectively. The effective porosity is 0.3 . Both the longitudinal and transverse dispersivities are $10 \mathrm{ft}$. The injection well is located in the middle of the model domain, i.e., at column 16, row 16 and layer 1.

MODFLOW was first used to simulate the steady-state flow field. The contour of the simulated hydraulic head consists of concentric circles (Figure 29(a)), as expected. In the center of the model domain, the contour however exhibits a slightly irregular shape, probably due to the small area of the circle relative to the grid size. RWHet was then used to calculate the streamlines (Figure 29(b)), which show the divergence of the flow field. The simulated particle clouds at various times (Figure 29(c)) also expand as concentric circles, as expected.

RWHet was finally applied to solve the transport model (1). The simulated plume snapshot generally matches the analytical solution (Figure 30(b)). The symmetric distribution of plumes around the injection well is also apparent (Figure 30(a)). 
(a) Contour of head

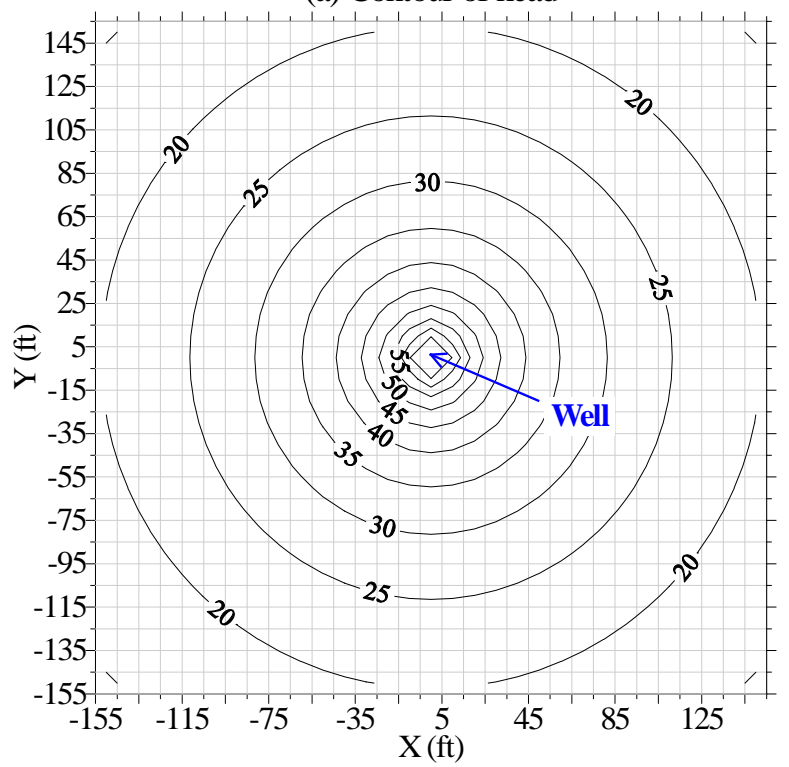

(b) Streamlines

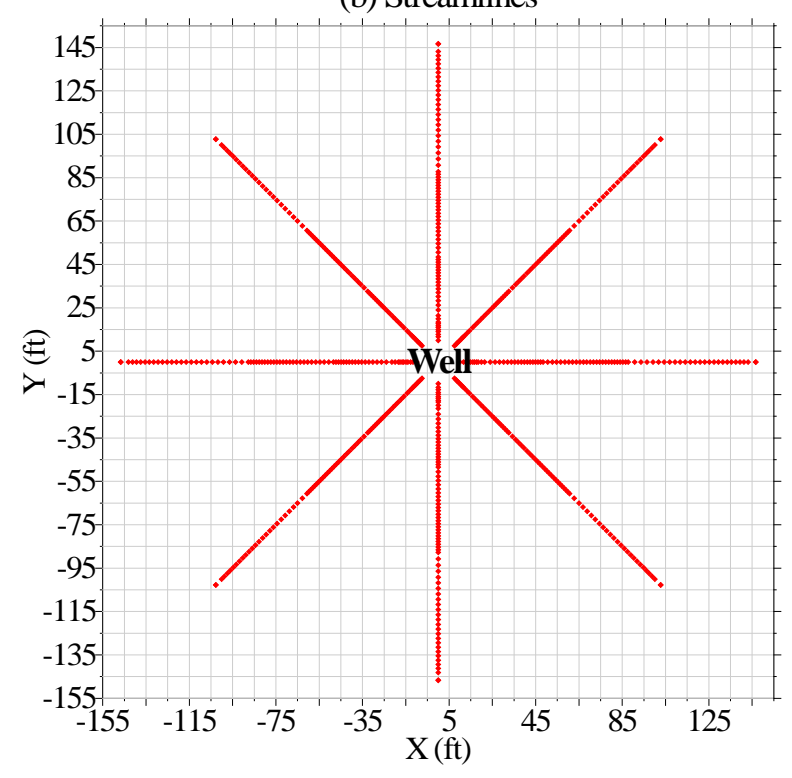

(c) Particle clouds at various times

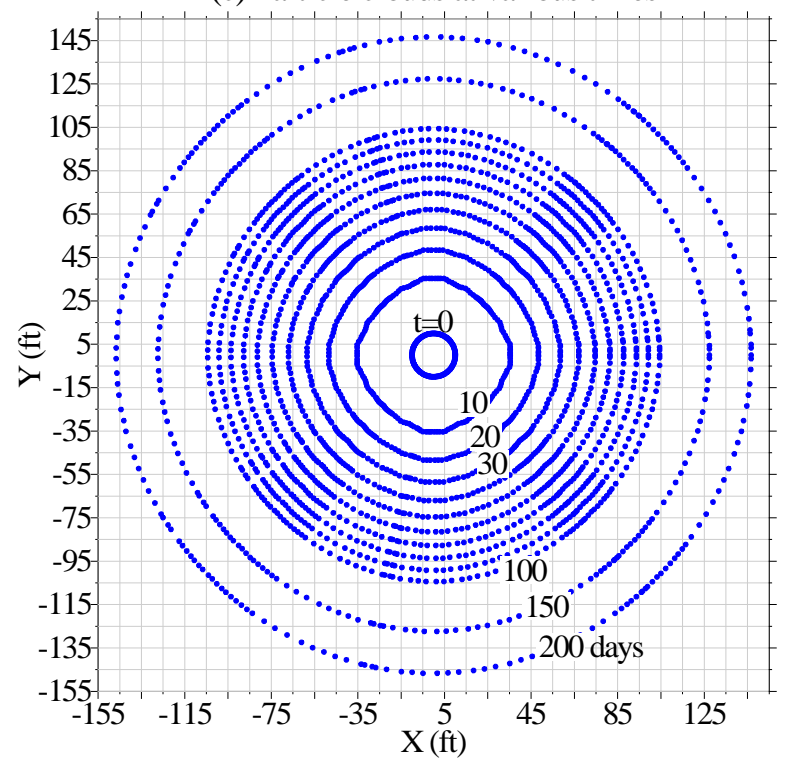

Figure 29. Example 2 - (a) shows the contour of hydraulic heads calculated by MODFLOW. (c) shows RWHet solutions of particle plumes at various times. The streamlines are shown in (b). The meshes at the background represent the actual model meshes (31 columns $\times 31$ rows). The pumping well is located at the middle grid of each figure. 


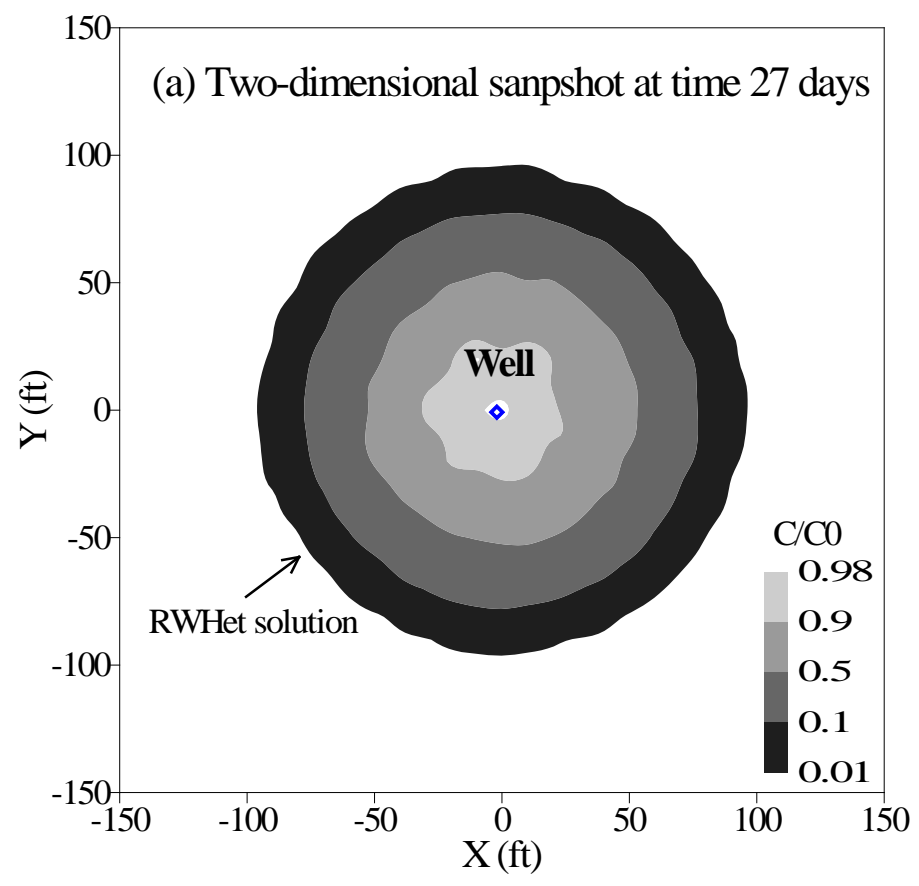

(b) Snapshot along the $\mathrm{X}$ axis

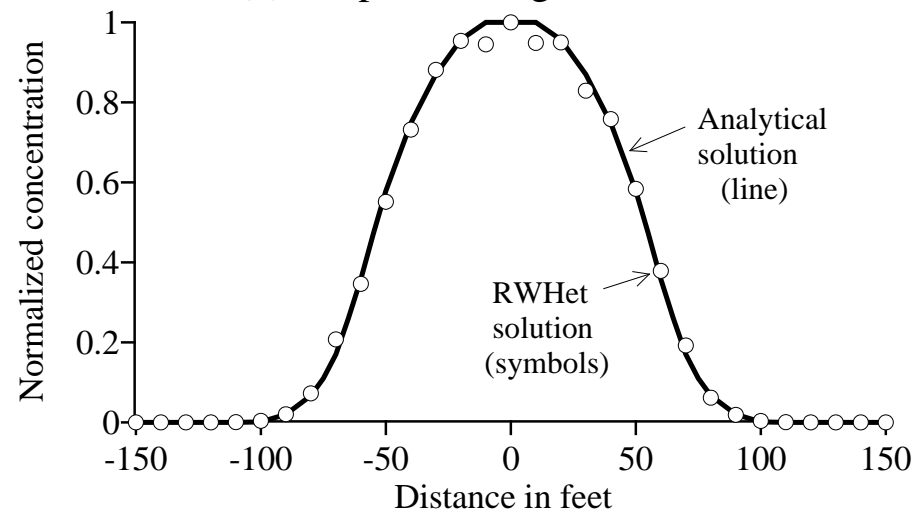

Figure 30. Example 2 - Two-dimensional transport in a radial flow field. (a) is the RWHet simulation of the 2-d snapshot at time $t=27$ days. (b) shows the comparison of the tracer snapshot along the X-axis using RWHet (symbols) and the analytical solutions (line). Adopted from Zheng and Wang [1999]. The average difference between the numerical solution and the analytical solution is $-4.57 \times 10^{-3}$. 
Example 3 - This example involves solute transport in a 2- $d$ strongly diverging/converging and transient flow field. It contains an injection/pumping cycle for a fully penetrating well in a confined aquifer. The details of this example can be seen in Zheng and Wang [1999]. The numerical model consists of 31 columns, 31 rows and 1 layer, with cell size $900 \times 900 \times 20 \mathrm{ft}$ along the column, row, and layer, respectively. The injection cycle (with the volumetric injection rate $1 \mathrm{ft}^{3} /$ second) is from $t=0 \sim 2.5 \mathrm{yrs}$ at the well located in the middle of the model domain (i.e., column 16 and row 16), followed by a pumping cycle with the same rate from 2.5 to 10 yrs. The effective porosity is 0.3 . The analytical solution of the concentration change at the injection/pumping well was given by Gelhar and Collins [1971]. Results (Figure 31) show that RWHet solutions generally match the analytical solution.

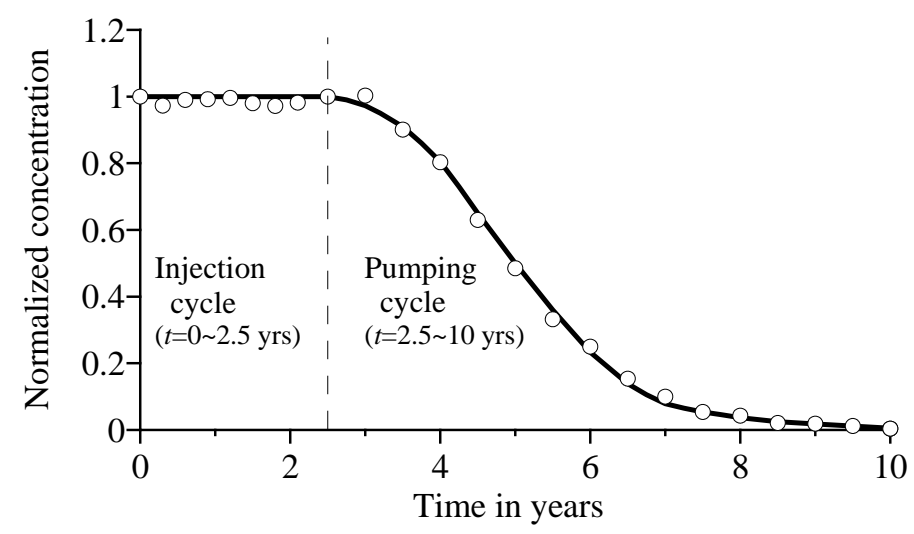

Figure 31. Example 3 - Concentration profiles (i.e., normalized concentration over time) at the well during an injection/pumping cycle. The symbols are the numerical solutions from RWHet, and the solid line denotes the analytical solution for solute transport in a strongly diverging/converging flow field. Adopted from Zheng and Wang [1999]. The average difference between the numerical solution and the analytical solution is $6.88 \times 10^{-4}$.

Figure 32 shows the particle locations at various times (simulated by RWHet) and the corresponding hydraulic head contour (solved by MODFLOW) at each cycle. The "circles" in Figure 32(a) and 32(b) are not smooth, due to the coarse-resolution model grid $(d x=d y=900 \mathrm{ft}$ ) and the irregular shape of the simulated head contour. Such behavior is different from Example 2 (Figure 29), where the relatively fine-resolution ( $d x=d y=10$ $\mathrm{ft}$ ) model grid results in a fine-resolution flow field and regular particle cloud. Note that RWHet efficiently captures the discrepancy of flow fields between Example 2 and Example 3, by interpolating the MODFLOW velocities correctly to obtain the velocity for each particle. This verifies further the reliability of RWHet. 
(a) Particle position (Injection cycle)

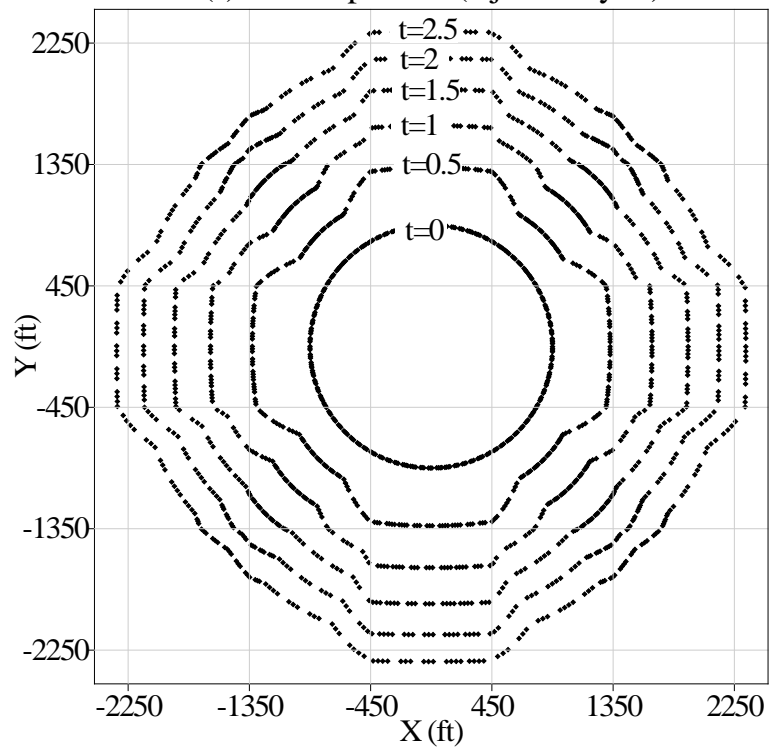

(c) Contour of head (Injection cycle)

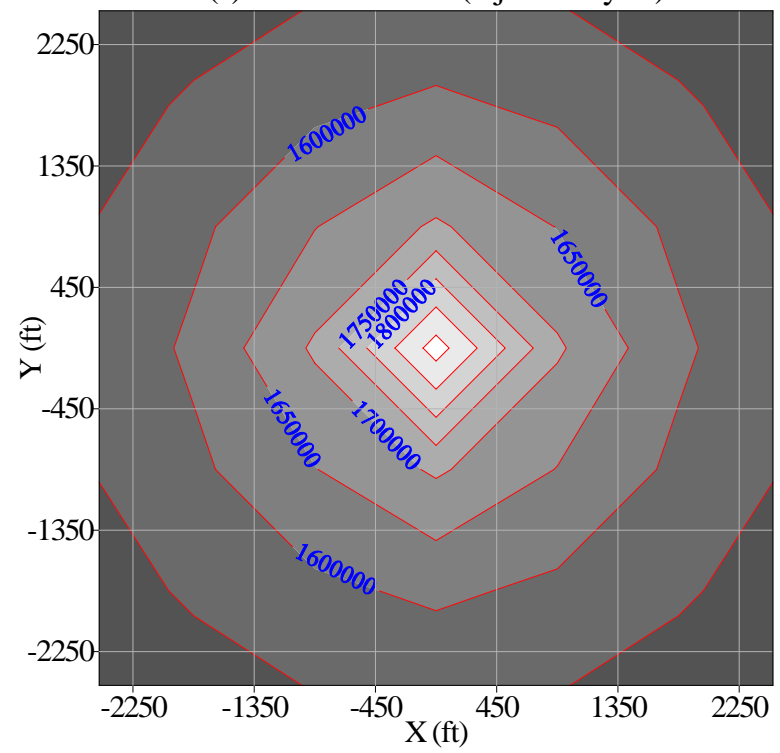

Head (ft)

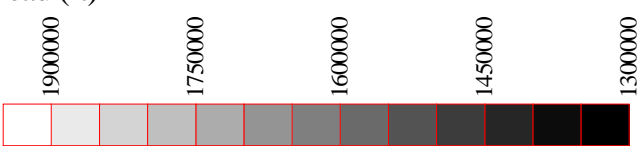

(b) Particle position (Pumping cycle)

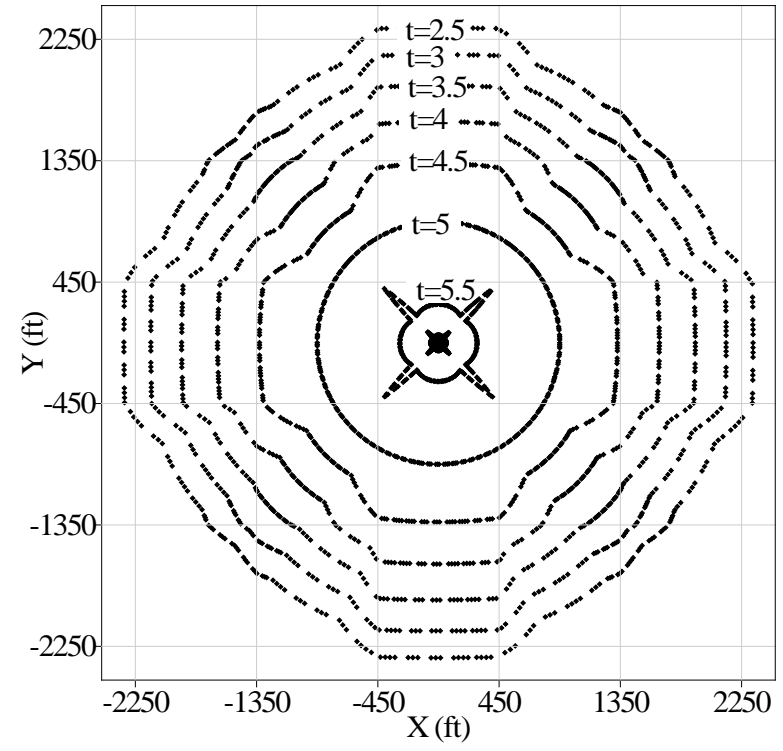

(d) Contour of head (Pumping cycle)

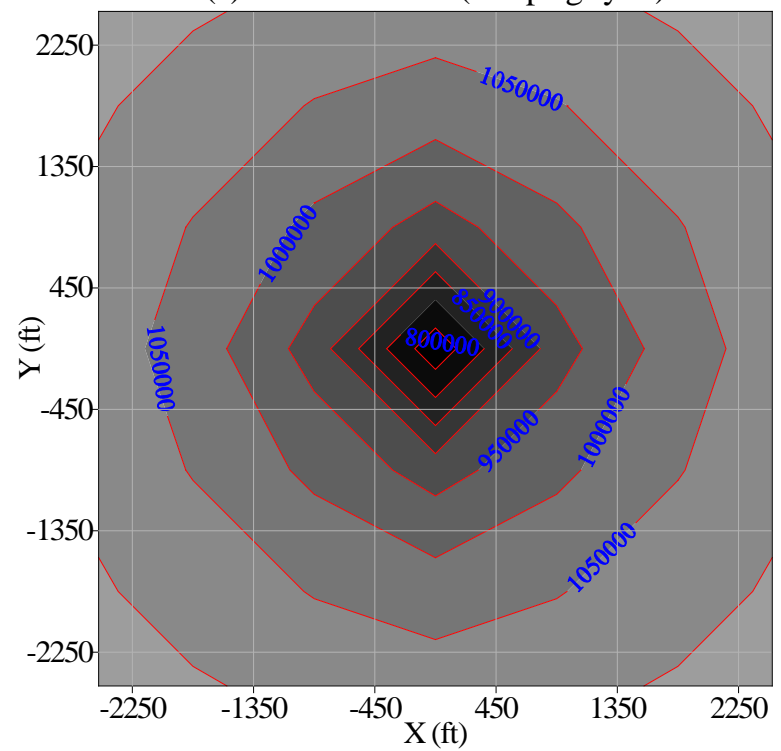

Head (ft)

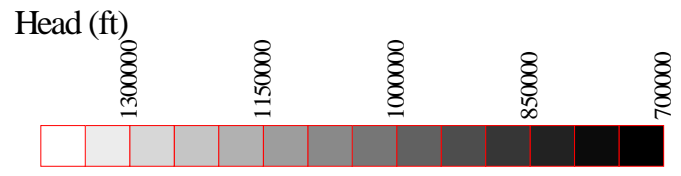

Figure 32. Example 3 - Particle positions (due to the advection-only transport) at various times during the injection cycle (a) and the pumping cycle (b). The hydraulic head at each cycle is also shown in (c) and (d). The meshes shown in (a) (d) represent the actual model meshes. 


\section{A.3. Two-dimensional heterogeneous box: Advection-only transport (Examples 4 and 5)}

We considered two boxes with a difference degree of "heterogeneity" (i.e., a progression of complexity). The first one (Example 4) was adopted from Zheng and Wang [1999, Chapter 7.9 - A two-dimensional application example], where a lowpermeable block is embedded in a relatively high-permeable material. The second case (Example 5) contains a more complex structure of hydraulic conductivity distributions, representing a high-resolution, "strongly" heterogeneous medium with abrupt interfaces of distinct deposits observed in the field. The two cases have quite different grid resolutions, and they can be used to test the capability of a Lagrangian solver in capturing the influence of sharp-contrasts in velocity on particle dynamics. This section discusses the advection-only transport. The influence of dispersion will be discussed in the following.

\section{A.3.1. Example 4}

The flow and transport model setup was described in detail by Zheng [1992]. For the convenience of readers, here we introduce them briefly. This example involves flow and transport in a 2- $d$ heterogeneous aquifer, where the analytical solutions are not available. The flow model configuration is shown in Figure 33(a). Water is injected in to the aquifer through a fully penetrating well at the north (near the upstream boundary), and a pumping well is located at the south. Between these two wells, there is a lowpermeability zone.

RWHet was used to evaluate how the streamline changes with the injection and pumping rates in the heterogeneous aquifer. When the injection and pumping rates are relatively small (i.e., Figure 33(b)), neither the flow fields (solved by MODFLOW) nor the streamlines are affected apparently. With the increase of injection and pumping rates, the divergence near the injection well and the convergence near the pumping well are enhanced for both flow and transport (Figure 33(c), (d)). 

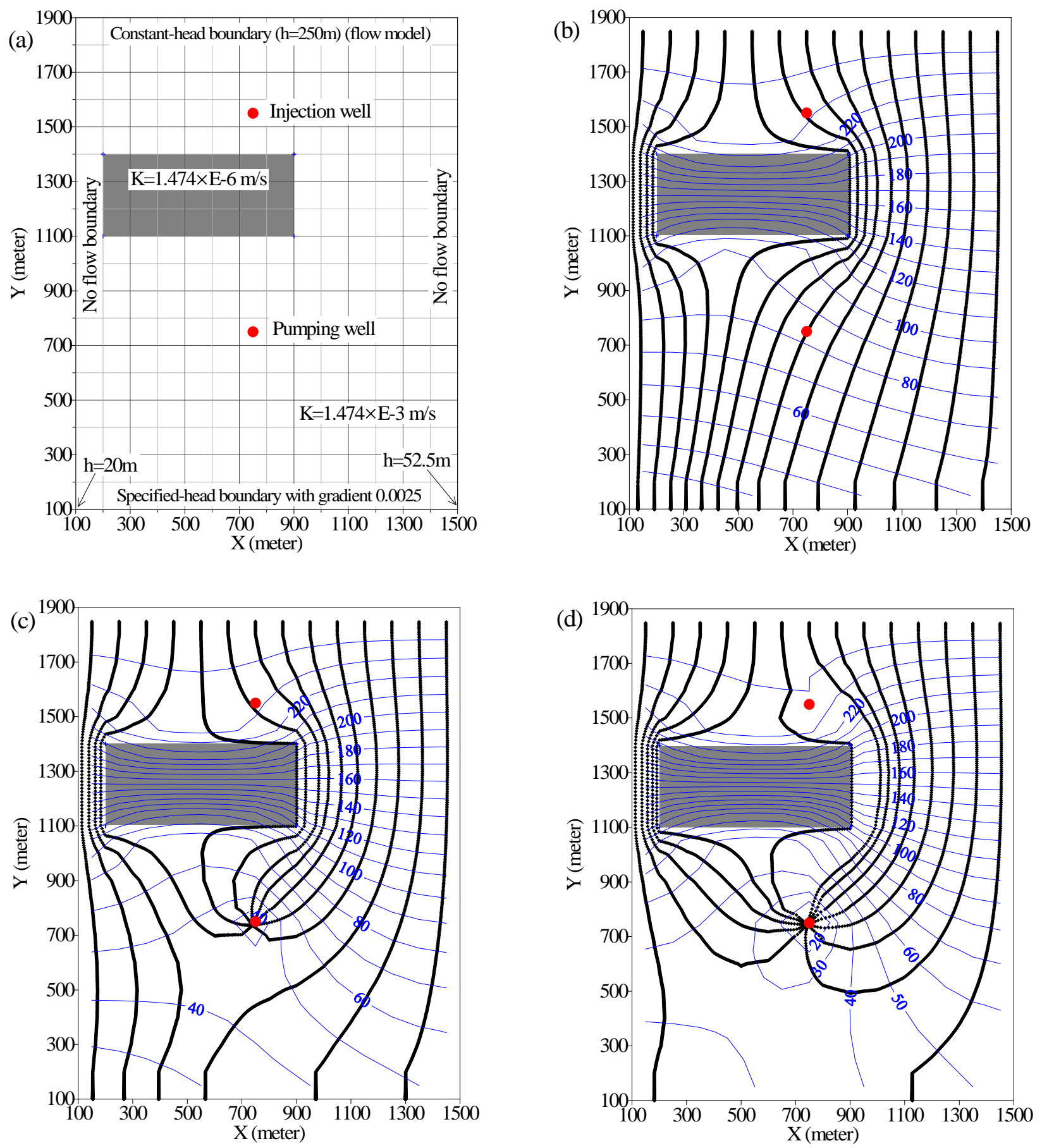

Figure 33. Example 4 - (a) Configuration of the flow model in a heterogeneous aquifer with wells. (b), (c) and (d) show the simulated streamlines (black lines) using RWHet, due to various injection and pumping rates. In (b), the injection rate is 0.001 $\mathrm{m}^{3} /$ second and the pumping rate is $0.0189 \mathrm{~m}^{3} / \mathrm{s}$. In (d), the injection rate is 0.0926 $\mathrm{m}^{3} / \mathrm{s}$ and the pumping rate is $1.16 \mathrm{~m}^{3} / \mathrm{s}$. In (c), the injection rate is $0.315 \mathrm{~m}^{3} / \mathrm{s}$ and the pumping rate is $1.89 \mathrm{~m}^{3} / \mathrm{s}$. In (b), (c) and (d), the blue lines denote the contours of hydraulic head (meter). Modified from Zheng and Wang [1999]. 


\section{A.3.2. Example 5}

This example shows the streamline (and its statistics) across a "strongly" heterogeneous media with random distributed hydraulic conductivities (Figure 34).

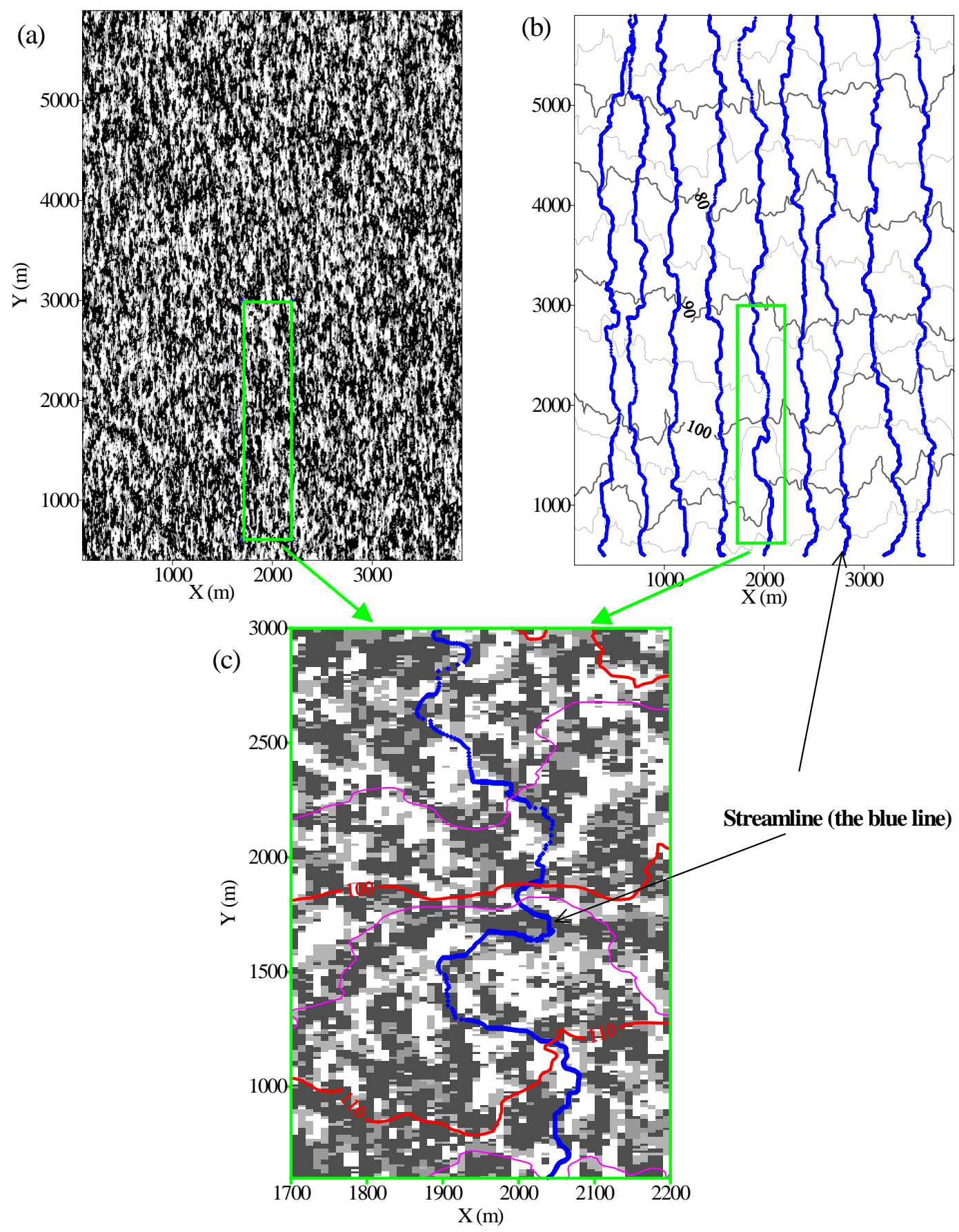

Figure 34. Example 5 - Particle tracking through a heterogeneous medium. (a) The distribution of hydrofacies generated by T-PROGS [Carle, 1999]. (b) The simulated contour of hydraulic head using MODFLOW and the streamlines (blue lines) calculated by RWHet. (c) lists the detail in the green box shown in (a) and (b). In the images, the darker cell has a smaller hydraulic conductivity. 
The well-known code T-PROGS [Carle, 1999] was first used to generate the heterogeneous medium (Figure 33(a)), where the 2- $d$ Markov Chain model was adopted from Carle [1996] and LaBolle and Fogg [2001]. The 3-d cell size of 10, 10 and $10 \mathrm{~m}$ in the depositional strike (Y-axis), depositional dip (X-axis), and vertical directions was selected. The overall dimensions of the simulated region are $6010 \times 4010 \times 10 \mathrm{~m}(\mathrm{y} / \mathrm{x} / \mathrm{z})$, with $601 \times 401 \times 1=241,001$ cells. The same model and grid size were used in the flow and transport models.
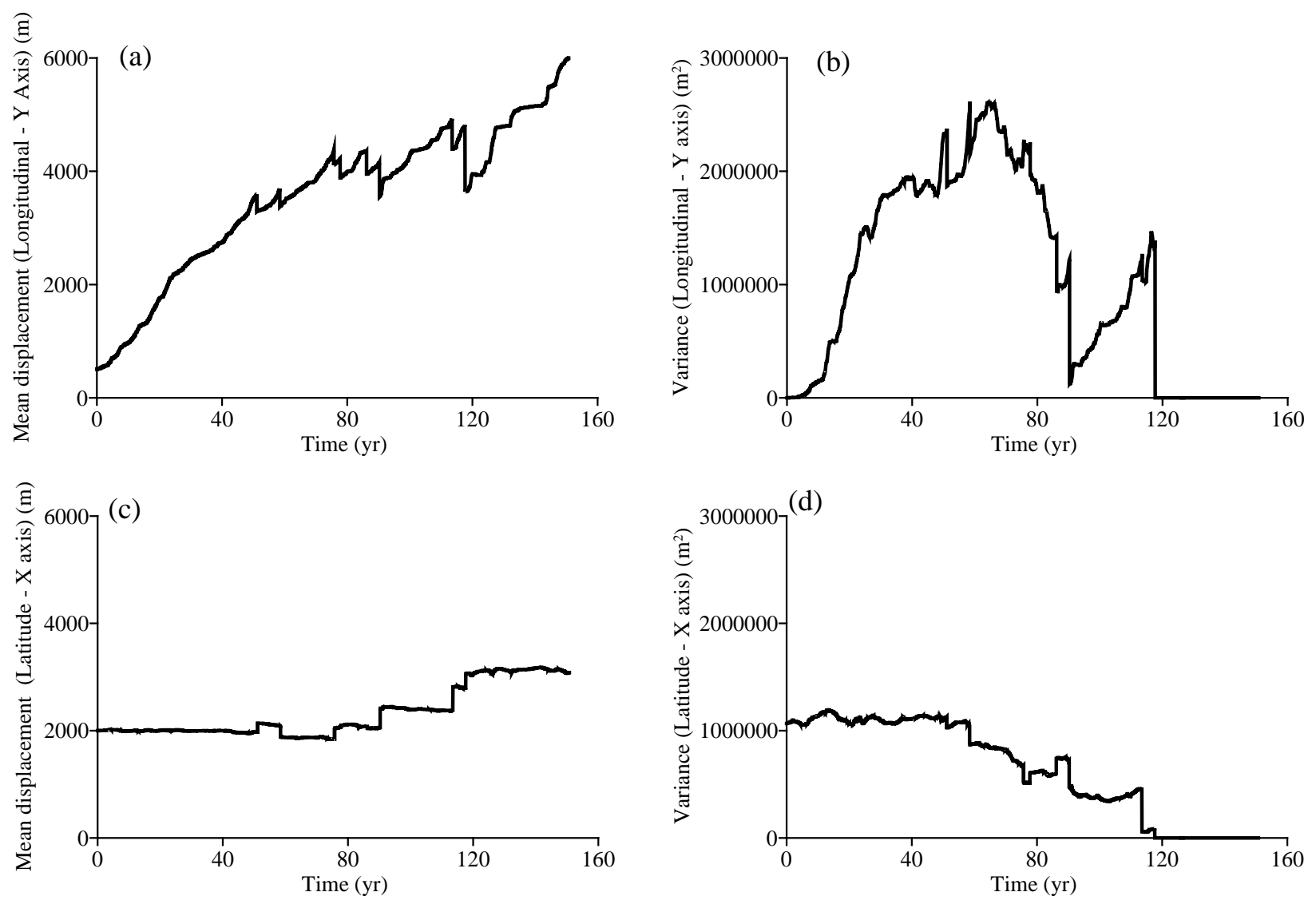

Figure 35. Example 5 - Statistics of particle tracking simulation (advection-only) using RWHet. (a) and (b) show the mean and variance of displacement for the 9 particles along the longitudinal direction (i.e., $Y$-axis shown in Figure 34). (c) and (d) are the same statistics along the horizontal direction (i.e., $X$-axis shown in Figure 34).

Figure 34 shows the simulated streamlines using RWHet. Figure 35 shows the statistics of particle trajectories (due to the advection-only transport). 


\section{A.4. Two-dimensional heterogeneous box: Advection \& dispersion (Examples 4 and 5)}

We first add dispersion in the transport process for Example 4 (Figure 36). The transport model set up is shown in Figure 36a.
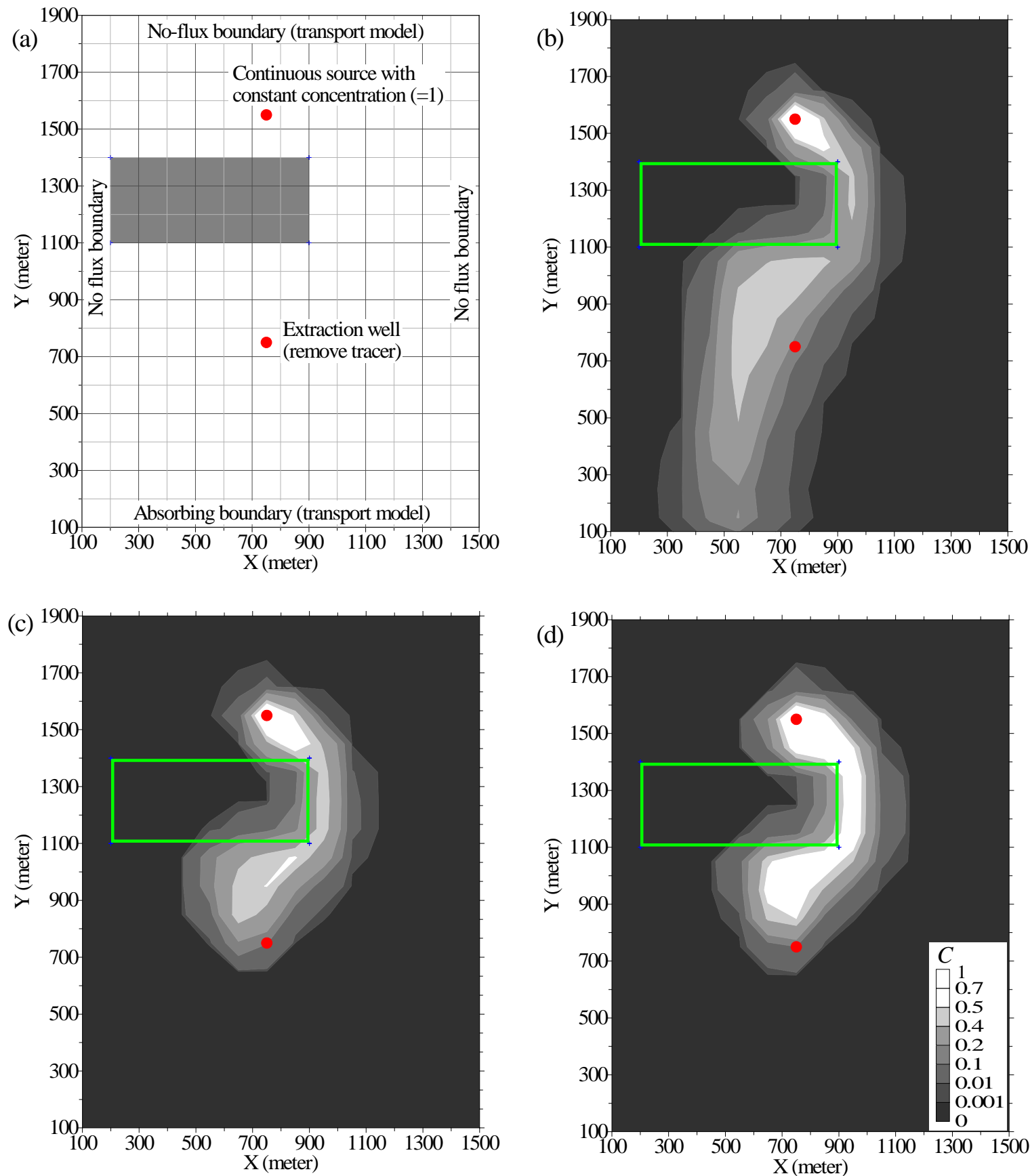

Figure 36. Example 4 - Distribution of solute concentration at time $t=36.5$ days as solved by RWHet. See also Zheng [1992, page 7-18] for a similar tracer snapshot as the one shown in (b). The flow field for (b), (c) and (d) is the same as Figure 32 (b), (c), and (d), respectively. 
The effective porosity is 0.3 . The longitudinal and transverse dispersivity are 6 $\mathrm{m}$ and $1 \mathrm{~m}$, respectively. The influence of molecular diffusion is neglected (for the comparison with MT3D in Zheng [1992]). A continuous source with a constant concentration $C=1$ is located at the injection well. The simulated plume snapshot for each flow field using RWHet is shown in Figure 36(b), 36(c), and 36(d).

We then add dispersion in the transport process for Example 5 (Figure 37). The effective porosity is 0.3 . The longitudinal and transverse dispersivity are $4 \mathrm{~m}$ and $0.4 \mathrm{~m}$, respectively. The molecular diffusion coefficient is $5.2 \times 10^{-4} \mathrm{~m}^{2} /$ day. A continuous source with a constant concentration $C=1$ is located near the upstream boundary. The simulated plume snapshots at various times using RWHet are shown in Figure 37.
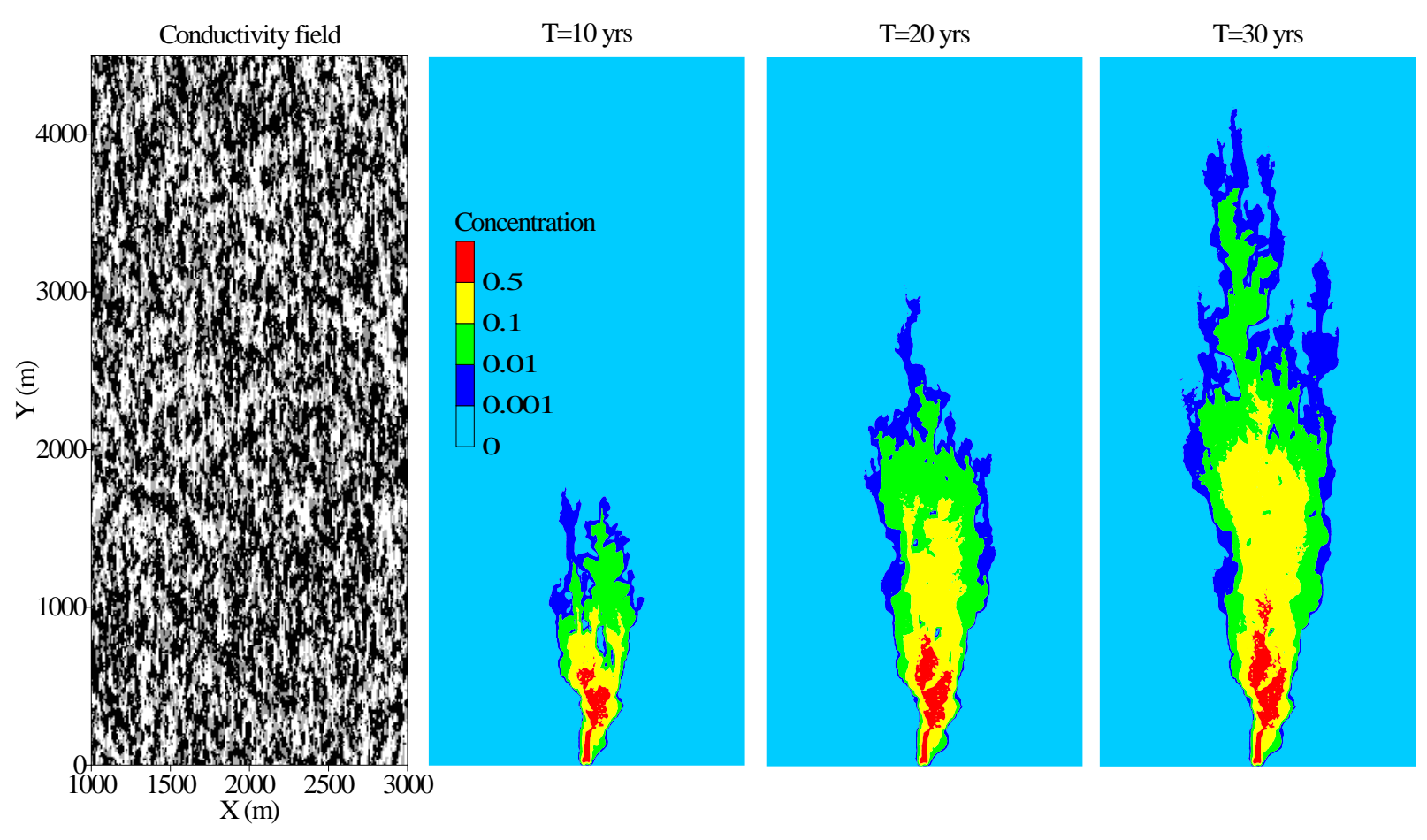

Figure 37. Example 5 - Time evolution of solute particles (snapshots) through the strong heterogeneous flow field (same as Figure 34). 


\section{A.5. Walkabout Verification Test 3 (Example 6)}

Example 6 - This example is analogous to the Verification Test 3 in Painter [2011, Figure 10], a 2- $d$ flow and transport through a composite medium. The model domain is $100 \times 100 \times 100 \mathrm{~m}$, with a structured (uniform) grid size $2 \times 2 \times 10 \mathrm{~m}$ for each column, row and layer. The whole domain has a hydraulic conductivity $(K) 6.49 \times 10^{-2}$ $\mathrm{m} /$ day, except for two regions (shown by the shaded areas in Figure 38(b)) with a lower $K=6.49 \times 10^{-7} \mathrm{~m} /$ day. In the flow model, the left and right boundaries are constant-head boundaries, with hydraulic head of 90 and 70 meters, respectively. The top and bottom boundaries are no flow boundaries. In the transport model, all boundaries are reflective (i.e., no flux) boundaries except for the right one, which is an absorbing boundary. The effective porosity is 0.3 .

Trajectories calculated by RWHet are shown in Figure 38(a). Figure 38(b) shows the Walkabout results [Painter, 2011, Figure 10]. RWHet results generally match the trend of the Walkabout streamlines.
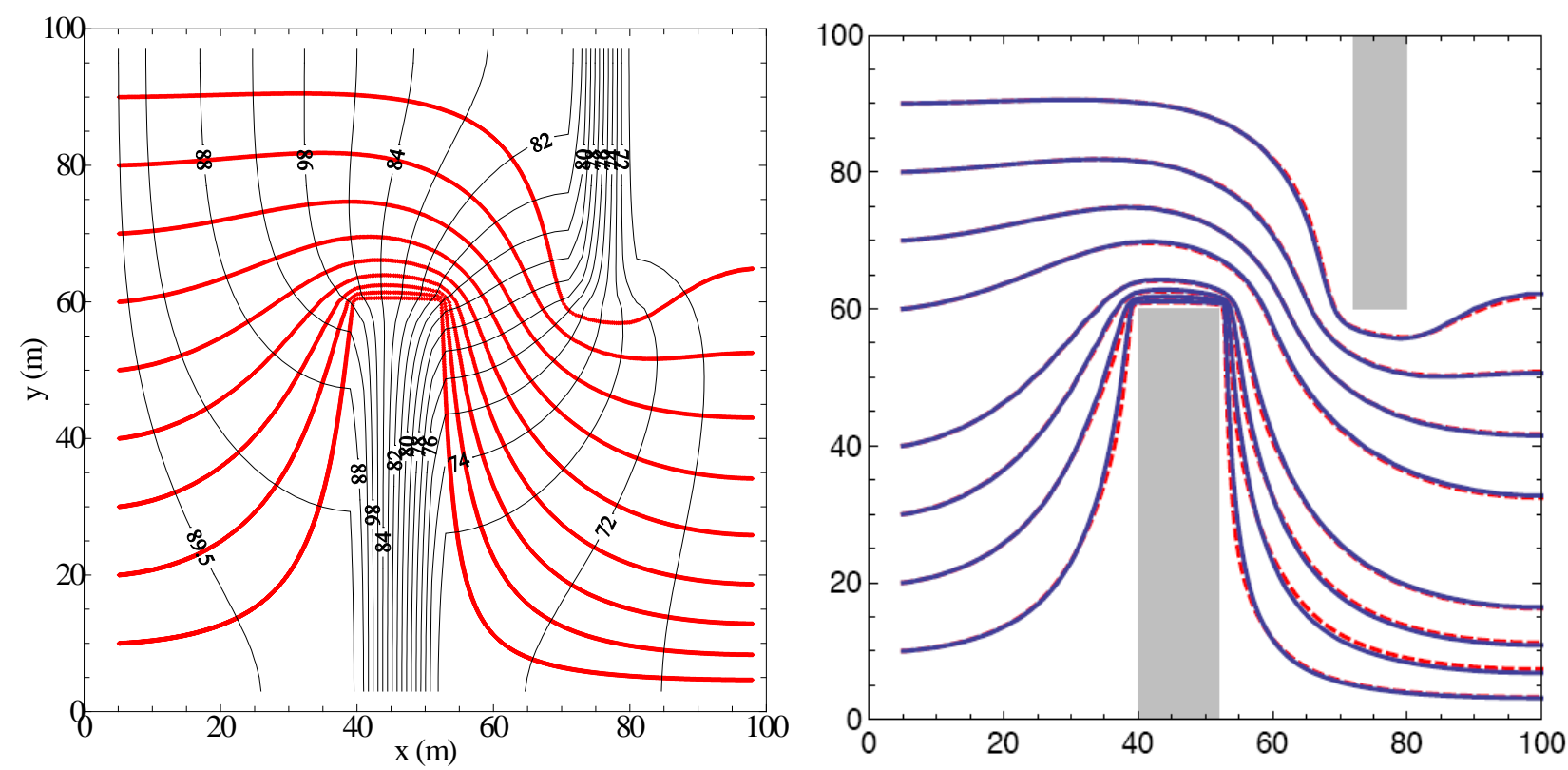

Figure 38. Example 6 - Left: The contour of the hydraulic heads simulated by MODFLOW (black lines) and the streamlines calculated by RWHet (red lines). Note here we have 9 streamlines, while Painter [2011, Figure 10] (the right figure) showed only 8 of them (without the middle one). Right: Copy from Painter [User's Manual for Walkabout Version 1.0, 2011, Figure 10, page 14] - Results of Verification Test 3. Shown are horizontal projections of streamlines calculated by SPTR (solid blue) and Walkabout (red dashed).

All of the above six examples show that RWHet captures the streamlines and solute transport reliably in 1- $d$ and multiple dimensions. 